\title{
Substrate Specific Metal-Ligand Cooperative Binding: Considerations for Weak Intramolecular Lewis Acid/Base Pairs
}

\author{
John J. Kiernicki, ${ }^{a \ddagger}$ Emily E. Norwine, ${ }^{a \ddagger}$ Matthias Zeller, ${ }^{b}$ and Nathaniel K. Szymczak ${ }^{a *}$
}

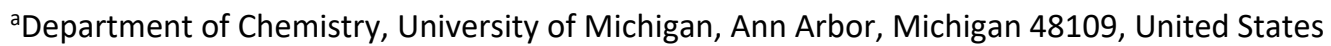

${ }^{\mathrm{b} H . C .}$ Brown Laboratory, Department of Chemistry, Purdue University, West Lafayette, Indiana 47907, United States

${ }^{\ddagger}$ These authors contributed equally.
} 
Experimental Procedures.....

S3-S6

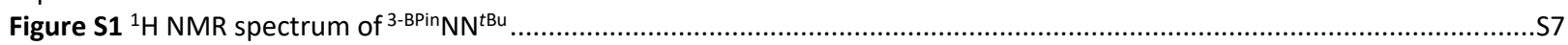

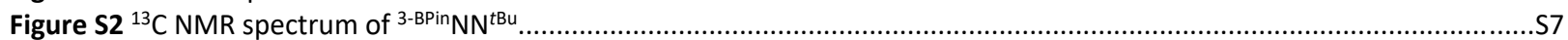

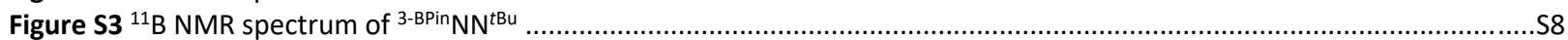

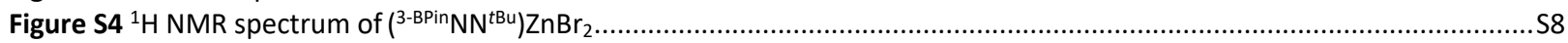

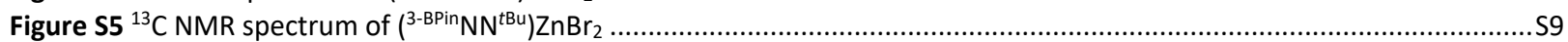

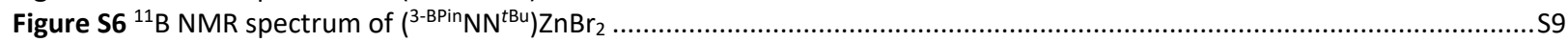

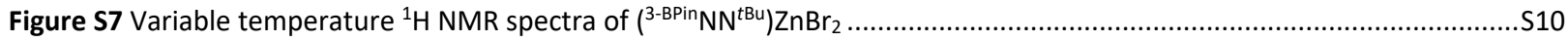

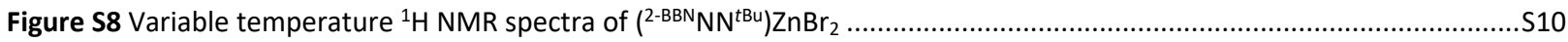

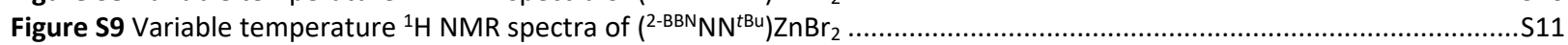

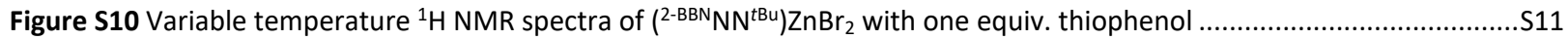

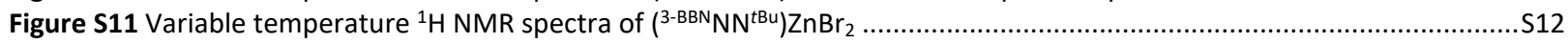

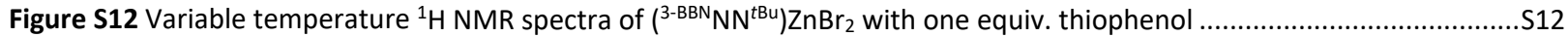

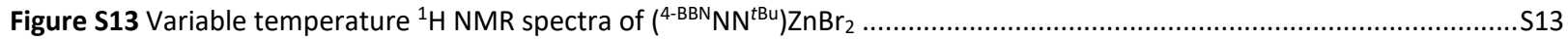

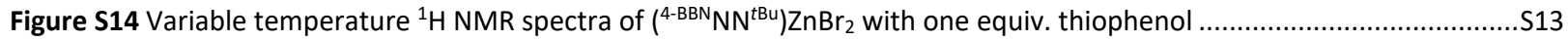

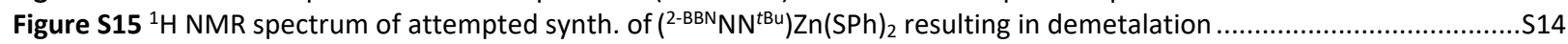

Figure $\mathbf{S 1 6}{ }^{1} \mathrm{H}$ NMR spectrum of attempted alternate synth. of $\left({ }^{2-\mathrm{BBN}} \mathrm{NN}^{t \mathrm{Bu}}\right) \mathrm{Zn}(\mathrm{SPh})_{2}$ resulting in demetalation .....................S14

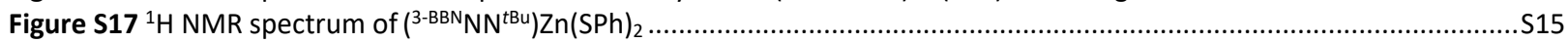

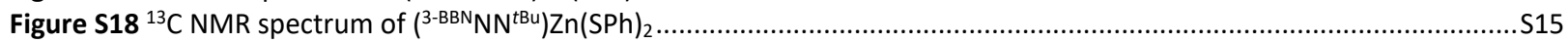

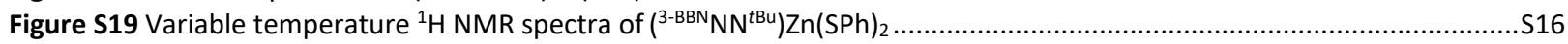

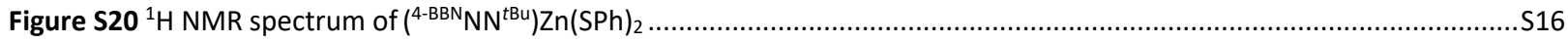

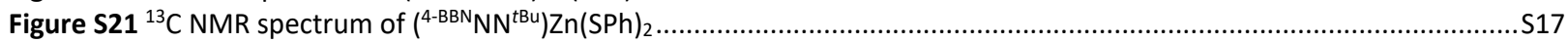

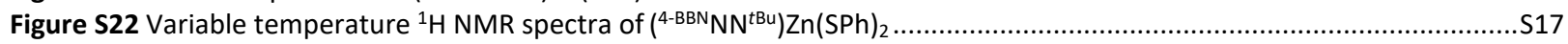

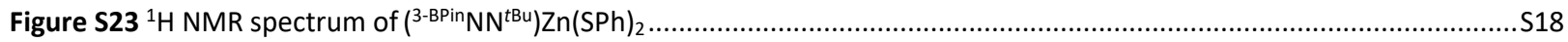

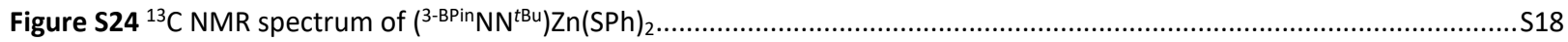

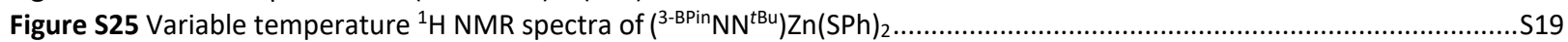

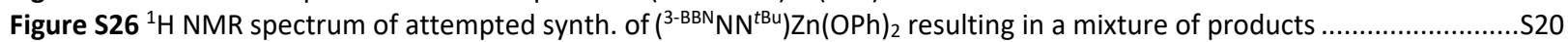

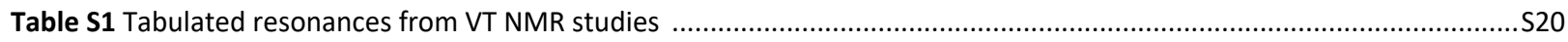

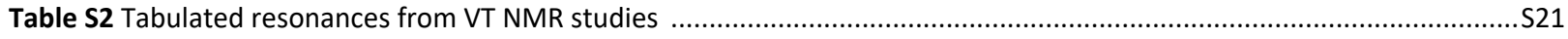

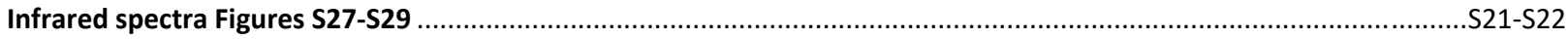

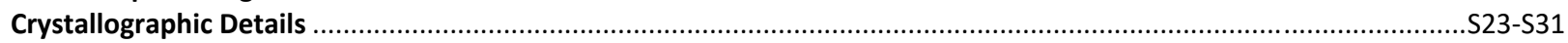

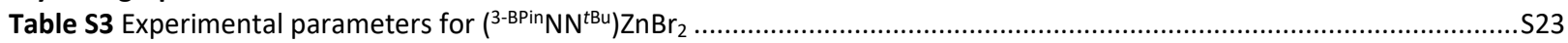

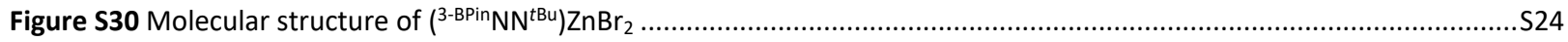

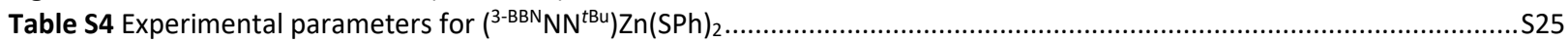

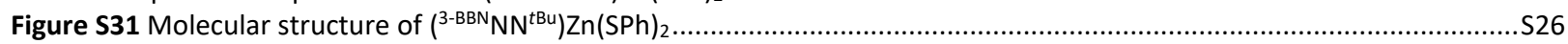

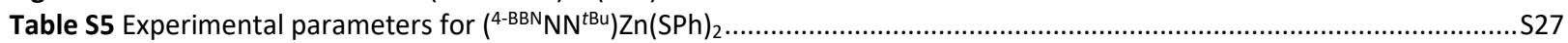

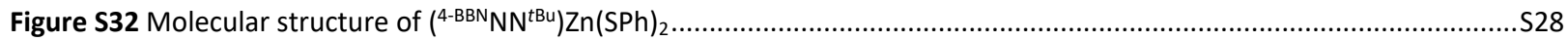

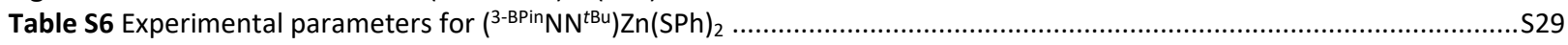

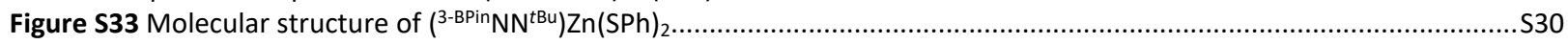

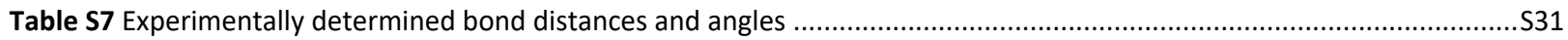

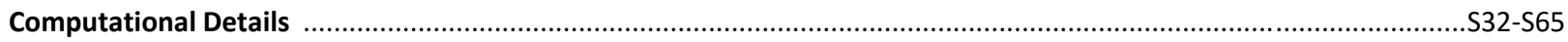

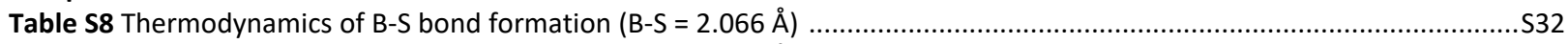

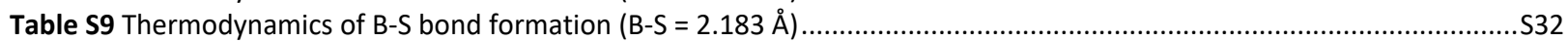

Table S10 Comparison between computationally and experimentally determined bond distances and angles .......................S33

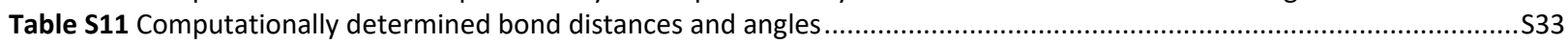

Figure S34 Structural overlay of DFT optimized geometries and molecular structures ....................................................S34

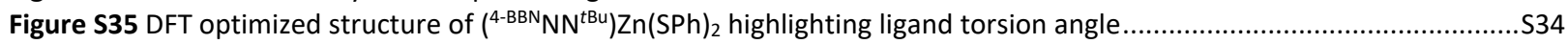

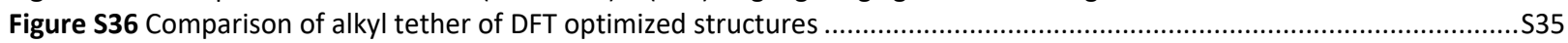

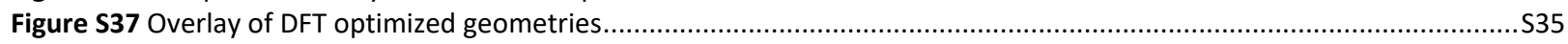

Figure S38 DFT optimized geometries of species with and without B-S interaction (side-by-side) .......................................S36

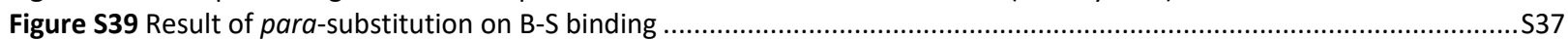

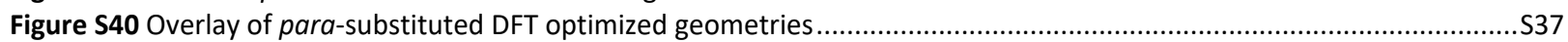

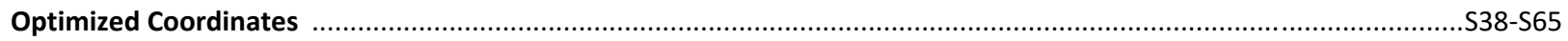

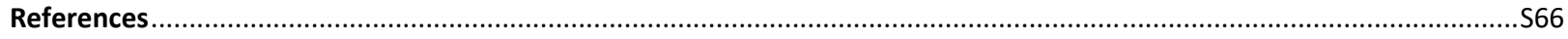


General Considerations. All air- and moisture-sensitive manipulations were performed using standard Schlenk techniques or in an inert atmosphere drybox with an atmosphere of purified nitrogen. The drybox was equipped with a cold well designed for freezing samples in liquid nitrogen as well as a $-35^{\circ} \mathrm{C}$ freezer for cooling samples and crystallizations. Solvents were purified using a Glass Contour solvent purification system through percolation through a $\mathrm{Cu}$ catalyst, molecular sieves, and alumina. Solvents were then stored over sodium and/or sieves. Benzene- $d_{6}$, dichloromethane- $d_{2}$, and chloroform- $d$ were purchased from Cambridge Isotope Laboratories. Benzene- $d_{6}$ was dried with molecular sieves and sodium, and degassed by three freeze-pump-thaw cycles. Chloroform- $d$ and dichloromethane- $d_{2}$ were distilled from $\mathrm{CaH}_{2}$.

Zinc(II) bromide, $\mathrm{Nal}, \mathrm{NaH}$, thiophenol, and potassium bis(trimethylsilylamide) were purchased from commercial vendors and used as received. ${ }^{H} \mathrm{NN}^{\mathrm{tBu}}, 1$ 2-(3-bromopropyl)-4,4,5,5-tetramethyl-1,3,2dioxaboralane, ${ }^{2}\left({ }^{\mathrm{n}-\mathrm{BBN}} \mathrm{NN}^{\mathrm{tBu}}\right) \mathrm{ZnBr}_{2}(\mathrm{n}=2,4),{ }^{3}$ and $\left({ }^{3-\mathrm{BBN}} \mathrm{NN}^{t \mathrm{Bu}}\right) \mathrm{ZnBr}_{2}{ }^{4}$ were synthesized according to literature procedures.

NMR spectra were recorded on Varian Vnmrs 700 or Varian MR400 spectrometers. ${ }^{1} \mathrm{H}$, ${ }^{13} \mathrm{C}$, and ${ }^{11} \mathrm{~B}$ chemical shifts are reported in parts per million (ppm) relative to tetramethylsilane and referenced internally to the residual solvent peak. ${ }^{11} \mathrm{~B}$ spectra were referenced on a unified scale, where the single primary reference is the frequency of the residual solvent peak in the ${ }^{1} \mathrm{H}$ NMR spectrum. ${ }^{11} \mathrm{~B}$ spectra are referenced vs. $\mathrm{BF}_{3}\left(\mathrm{OEt}_{2}\right)$. Multiplicities are reported as follows: singlet $(\mathrm{s})$, doublet $(\mathrm{d})$, triplet $(\mathrm{t})$, quartet (q). Infrared spectra were recorded using a Nicolet iS10 FT-IR spectrometer. Samples were diluted into dry $\mathrm{KBr}$ and recorded as pellets.

Single crystals of $\left({ }^{3-B B N} \mathrm{NN}^{t B u}\right) \mathrm{Zn}(\mathrm{SPh})_{2}$, $\left({ }^{4-B B N} \mathrm{NN}^{t \mathrm{Bu}}\right) \mathrm{Zn}(\mathrm{SPh})_{2},\left({ }^{3-B P i n} \mathrm{NN}^{t \mathrm{Bu}}\right) \mathrm{Zn}(\mathrm{SPh})_{2}$, and $\left({ }^{3-B P i n} \mathrm{NN}^{\mathrm{tBu}}\right) \mathrm{ZnBr}_{2}$ suitable for X-ray diffraction were coated with poly(isobutylene) oil and quickly transferred to the goniometer head of a Bruker AXS D8 Quest diffractometer with a fixed chi angle, a sealed tube fine focus $X$-ray tube, single crystal curved graphite incident beam monochromator and a Photon100 $\left(\left(^{3-}\right.\right.$ $\left.{ }^{B P i n} \mathrm{NN}^{t B u}\right) \mathrm{ZnBr}_{2}$, $\left.\left.\quad{ }^{3-B B N} \mathrm{NN}^{t B u}\right) \mathrm{Zn}(\mathrm{SPh})_{2}\right)$ or a Photonll CMOS area detector $\left(\left(^{4-B B N} \mathrm{NN}^{t B u}\right) \mathrm{Zn}(\mathrm{SPh})_{2}, \quad{ }^{3-}\right.$ $\left.\left.{ }^{B P i n} \mathrm{NN}^{t \mathrm{tBu}}\right) \mathrm{Zn}(\mathrm{SPh})_{2}\right)$. Examination and data collection were performed with Mo Ka radiation $(\lambda=0.71073$ $\AA$ ). . Data were collected, reflections were indexed and processed, and the files scaled and corrected for absorption using APEX3, SAINT and SADABS. ${ }^{5}$ For all samples, the space groups were assigned using XPREP within the SHELXTL suite of programs ${ }^{6}$ and the structures were solved by direct methods using ShelXS- $97^{7}$ and refined by full matrix least squares against $F^{2}$ with all reflections using Shelx|2017 or Shelx|2018 ${ }^{8}$ using the graphical interface Shelxle. ${ }^{9}$ If not specified otherwise, $\mathrm{H}$ atoms attached to carbon atoms were positioned geometrically and constrained to ride on their parent atoms, with carbon hydrogen bond distances of $0.95 \AA$ for and aromatic $\mathrm{C}-\mathrm{H}, 1.00,0.99$ and $0.98 \AA$ for aliphatic $\mathrm{C}-\mathrm{H}, \mathrm{CH}_{2}$, and $\mathrm{CH}_{3}$ moieties, respectively. Methyl $\mathrm{H}$ atoms were allowed to rotate but not to tip to best fit the experimental electron density. $\mathrm{U}_{\text {iso }}(\mathrm{H})$ values were set to a multiple of $\mathrm{U}_{\text {eq }}(\mathrm{C})$ with 1.5 for $\mathrm{CH}_{3}$, and 1.2 for $\mathrm{CH}_{2}$, and $\mathrm{C}-\mathrm{H}$ units, respectively. Additional data collection and refinement details, including description of disorder (where present) can be found with the individual structure descriptions, below. Complete crystallographic data, in CIF format, have been deposited with the Cambridge Crystallographic Data Centre. CCDC 20734502073453 contains the supplementary crystallographic data for this paper. These data can be obtained free of charge from The Cambridge Crystallographic Data Centre via www.ccdc.cam.ac.uk/data_request/cif. 
Synthesis of 2-(5-(tert-butyl)-1-(3-(4,4,5,5-tetramethyl-1,3,2-dioxaborolan-2-yl)propyl)-1H-pyrazol-3yl)-6-methylpyridine ( $\left.{ }^{3-B P i n} \mathbf{N N}^{t B u}\right)$. Inside a glovebox, a $250 \mathrm{~mL}$ Schlenk flask was charged with sodium hydride $(0.102 \mathrm{~g}, 4.250 \mathrm{mmol})$ and $60 \mathrm{~mL}$ THF. The flask was removed from the glovebox and attached to a Schlenk line and chilled to $-78{ }^{\circ} \mathrm{C}$. While stirring and against a positive $\mathrm{N}_{2}$ flow, ${ }^{\mathrm{H}} \mathrm{NN}^{\mathrm{tBu}}(0.700 \mathrm{~g}, 3.251$ $\mathrm{mmol}$ ) was added. The flask was resealed with a septum containing a gas outlet. The reaction was slowly warmed to room temperature and stirred until all gas evolution ceased. With stirring, sodium iodide (0.487 g, $3.249 \mathrm{mmol}$ ) and 2-(3-bromopropyl)-4,4,5,5-tetramethyl-1,3,2-dioxaboralane (1.050 g, 4.218 mmol) were added against a positive flow of $\mathrm{N}_{2}$. A reflux condenser containing a gas outlet was attached and the reaction mixture was heated to reflux for $16 \mathrm{hrs}$. The solution was cooled to room temperature and volatiles were removed in vacuo. The material was dissolved in $100 \mathrm{~mL}$ chloroform and filtered through Celite and concentrated to a tan sludge. The material was purified by column chromatography on silica $\left(R_{f}=0.53 ; 2: 1\right.$ hexane:ethyl acetate), and dried to afford a colorless oil $(0.390 \mathrm{~g}, 1.017 \mathrm{mmol}, 31 \%)$ assigned as 2-(5-(tert-butyl)-1-(3-(4,4,5,5-tetramethyl-1,3,2-dioxaborolan-2-yl)propyl)-1H-pyrazol-3-yl)-6methylpyridine ( $\left.{ }^{3-\mathrm{BPin}} \mathrm{NN}^{t \mathrm{Bu}}\right)$. High-Res $\mathrm{MS}$ of $\mathrm{C}_{22} \mathrm{H}_{34} \mathrm{~N}_{3} \mathrm{O}_{2} \mathrm{~B}_{1}+\mathrm{H}$ : Calc. 384.2822; Found 384.3144. ${ }^{1} \mathrm{H} \mathrm{NMR}$ $\left(\mathrm{CDCl}_{3}, 25^{\circ} \mathrm{C}\right) \delta=0.90\left(\mathrm{t}, J=7.5,2 \mathrm{H}, \mathrm{B}-\mathrm{CH}_{2}\right), 1.24\left(\mathrm{~s}, 12 \mathrm{H}, \mathrm{BPin}-\mathrm{CH}_{3}\right), 1.41\left(\mathrm{~s}, 9 \mathrm{H}, \mathrm{C}\left(\mathrm{CH}_{3}\right)_{3}\right), 2.07$ (p, J = 7.8, $\left.2 \mathrm{H}, \mathrm{CH}_{2} \mathrm{CH}_{2} \mathrm{CH}_{2}\right), 2.57\left(\mathrm{~s}, 3 \mathrm{H}, \mathrm{CH}_{3}\right), 4.20\left(\mathrm{t}, J=7.8,2 \mathrm{H}, \mathrm{NCH}_{2}\right), 6.60(\mathrm{~s}, 1 \mathrm{H}$, pyrazole- $\mathrm{CH}), 7.00(\mathrm{~d}, \mathrm{~J}=7.4,1 \mathrm{H}$, pyridine- $\mathrm{CH}), 7.54(\mathrm{t}, \mathrm{J}=7.6,1 \mathrm{H}, p$-pyridine- $\mathrm{CH}), 7.70(\mathrm{~d}, \mathrm{~J}=7.6,1 \mathrm{H}$, pyridine- $\mathrm{CH}) .{ }^{13} \mathrm{C} \mathrm{NMR}\left(\mathrm{CDCl}_{3}, 25{ }^{\circ} \mathrm{C}\right)$

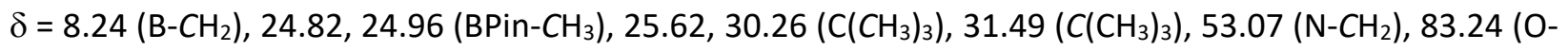
C), 101.72 (pyrazole- $\mathrm{CH}$ ), 117.07, 121.56, 136.61, 149.87, 152.36, 152.46, 157.99. ${ }^{11} \mathrm{~B} \mathrm{NMR}\left(\mathrm{CDCl}_{3}, 25{ }^{\circ} \mathrm{C}\right)$ $\delta=33.84$. Selected IR data (ATR, neat) $v=2974,1740,1594,1576,1369,1322,1143,967,845,794 \mathrm{~cm}^{-1}$.

Synthesis of $\left.{ }^{3-B P i n} \mathbf{N N}^{t B u}\right) Z_{n B r}$. A $20 \mathrm{~mL}$ scintillation vial was charged with zinc bromide $(0.215 \mathrm{~g}, 0.955$ $\mathrm{mmol}),{ }^{3-B P i n} \mathrm{NN}^{t \mathrm{Bu}}(0.358 \mathrm{~g}, 0.934 \mathrm{mmol})$, and $12 \mathrm{~mL}$ dichloromethane. The reaction was stirred for $48 \mathrm{hrs}$ at room temperature, filtered, and dried. The material was washed with $2 \times 10 \mathrm{~mL} n$-pentane and dried to afford a white powder $(0.531 \mathrm{~g}, 0.873 \mathrm{mmol}, 93 \%)$ assigned as $\left({ }^{3-B P i n} \mathrm{NN}^{\mathrm{tBu}}\right) \mathrm{ZnBr}_{2}$. Single, X-ray quality crystals were obtained by layering a tetrahydrofuran solution of $\left({ }^{3-\mathrm{BPin}} \mathrm{NN}^{\mathrm{tBu}}\right) \mathrm{ZnBr}_{2}$ with $n$-pentane at room temperature. High-Res MS of $\mathrm{C}_{22} \mathrm{H}_{34} \mathrm{~N}_{3} \mathrm{O}_{2} \mathrm{Br}_{2} \mathrm{~B}_{1} \mathrm{Zn}_{1}-\mathrm{Br}$ : Calc. 526.1219; Found 526.1214. ${ }^{1} \mathrm{H} \mathrm{NMR}\left(\mathrm{CDCl}_{3}\right.$, $\left.25{ }^{\circ} \mathrm{C}\right) \delta=1.00\left(\mathrm{t}, J=7.0,2 \mathrm{H}, \mathrm{B}-\mathrm{CH}_{2}\right), 1.22\left(\mathrm{~s}, 12 \mathrm{H}, \mathrm{BPin}-\mathrm{CH}_{3}\right), 1.47\left(\mathrm{~s}, 9 \mathrm{H}, \mathrm{C}\left(\mathrm{CH}_{3}\right)_{3}\right), 2.32(\mathrm{p}, J=8.0,2 \mathrm{H}$, $\left.\mathrm{CH}_{2} \mathrm{CH}_{2} \mathrm{CH}_{2}\right), 2.92\left(\mathrm{~s}, 3 \mathrm{H}, \mathrm{CH}_{3}\right), 4.46\left(\mathrm{t}, J=8.5,2 \mathrm{H}, \mathrm{NCH}_{2}\right), 6.55(\mathrm{~s}, 1 \mathrm{H}$, pyrazole- $\mathrm{CH}), 7.33(\mathrm{~d}, J=7.7,1 \mathrm{H}$, pyridine- $\mathrm{CH}), 7.60(\mathrm{~d}, J=7.8,1 \mathrm{H}$, pyridine- $\mathrm{CH}), 7.89(\mathrm{~d}, J=7.8,1 \mathrm{H}, p$-pyridine- $\mathrm{CH}) .{ }^{13} \mathrm{C} \mathrm{NMR}\left(\mathrm{CDCl}_{3}, 25{ }^{\circ} \mathrm{C}\right)$

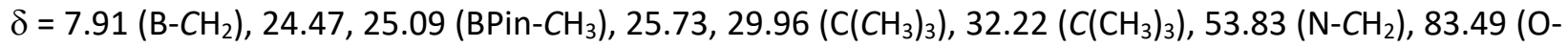
C), 101.69 (pyrazole-CH), 118.09, 125.74, 141.01, 145.58, 146.59, 156.98, 159.55. $\left.{ }^{11} \mathrm{~B} \mathrm{NMR}^{(\mathrm{CDCl}}{ }_{3}, 25{ }^{\circ} \mathrm{C}\right)$ $\delta=33.72$. Selected IR data (ATR, neat) $v=3127,1610,1572,1480,1372,1324,1227,1144,1022,844$, $809 \mathrm{~cm}^{-1}$.

\section{Attempted Synthesis of $\left(^{2-B B N} N^{t B u}\right) Z n(S P h)_{2}$.}

1) A $20 \mathrm{~mL}$ scintillation vial was charged with (2-BBN $\left.\mathrm{NN}^{\mathrm{tBu}}\right) \mathrm{ZnBr}_{2}(0.080 \mathrm{~g}, 0.136 \mathrm{mmol})$, thiophenol $(28 \mu \mathrm{L}$, $0.272 \mathrm{mmol}$ ) and $7 \mathrm{~mL}$ THF and frozen. A separate vial was charged with potassium bis(trimethylsilyl)amide $(0.054 \mathrm{~g}, 0.272 \mathrm{mmol})$ and $5 \mathrm{~mL}$ THF and frozen. Upon thawing, the solution of $\mathrm{KN}\left(\mathrm{SiMe}_{3}\right)_{2}$ was added dropwise to the solution containing $\left({ }^{3-\mathrm{BBN}} \mathrm{NN}^{\mathrm{tBu}}\right) \mathrm{ZnBr}_{2}$ and stirred while warming up to room temperature for $30 \mathrm{~min}$. The solution was filtered through Celite and dried. The material was washed with $2 \times 10 \mathrm{~mL} n$-pentane and dried to afford a white powder assigned as the demetallated ligand, $\left({ }^{2-B B N} N^{t B u}\right) .{ }^{1} \mathrm{H} N M R\left(C_{6} \mathrm{D}_{6}, 25^{\circ} \mathrm{C}\right) \delta=0.88\left(\mathrm{~s}, 9 \mathrm{H}, \mathrm{C}\left(\mathrm{CH}_{3}\right)_{3}\right), 1.16(\mathrm{~s}$, broad, $2 \mathrm{H}, \mathrm{B}-\mathrm{CH}), 1.57(\mathrm{t}, J=7.0,2 \mathrm{H}, \mathrm{B}-$ $\left.\mathrm{CH}_{2}\right), 2.02\left(\mathrm{~m}, 8 \mathrm{H}, \mathrm{BBN}-\mathrm{CH}_{2}\right), 2.25\left(\mathrm{~m}, 4 \mathrm{H}, \mathrm{BBN}-\mathrm{CH}_{2}\right), 2.41\left(\mathrm{~s}, 3 \mathrm{H}, \mathrm{CH}_{3}\right), 3.93\left(\mathrm{t}, \mathrm{J}=7.0,2 \mathrm{H}, \mathrm{N}-\mathrm{CH}_{2}\right), 5.98(\mathrm{~s}$, 
$1 \mathrm{H}$, pyrazole- $\mathrm{CH}), 6.63(\mathrm{~d}, J=7.6,1 \mathrm{H}$, pyridine- $\mathrm{CH}), 7.00(\mathrm{t}, J=7.6,1 \mathrm{H}$, pyridine- $\mathrm{CH}), 7.07(\mathrm{~d}, J=7.6,1 \mathrm{H}$, pyridine- $\mathrm{CH}$ ).

2) This method was attempted in both $\mathrm{CH}_{2} \mathrm{Cl}_{2}$ and THF. The scales were identical and the reactions results were identical. Only the reaction with THF is discussed here. A $20 \mathrm{~mL}$ scintillation vial was charged with (2$\left.{ }^{B B N} \mathrm{NN}^{\mathrm{tBu}}\right) \mathrm{ZnBr}_{2}(0.022 \mathrm{~g}, 0.037 \mathrm{mmol})$, thiophenol $(7.7 \mu \mathrm{L}, 0.075 \mathrm{mmol})$ and $4 \mathrm{~mL}$ THF. While stirring, neat $\mathrm{N}, \mathrm{N}$-diisopropylethylamine $(13.0 \mu \mathrm{L}, 0.075 \mathrm{mmol})$ was added dropwise to the solution and stirred for 24 $\mathrm{hr}$. Volatiles were removed in vacuo. The material was dissolved in $0.8 \mathrm{~mL} \mathrm{C}_{6} \mathrm{D}_{6}$ and filtered into a J Young NMR tube for analysis. ${ }^{1} \mathrm{H}$ NMR spectroscopy revealed only the presence of the demetallated ligand, $\left({ }^{2-}\right.$ ${ }^{\mathrm{BBN}} \mathrm{NN}^{\mathrm{tBu}}$ ), in solution.

Synthesis of $\left({ }^{3-B B N} N^{t B u}\right) Z n(S P h)_{2}$. A $20 \mathrm{~mL}$ scintillation vial was charged with $\left({ }^{3-B B N} \mathrm{NN}^{t B u}\right) \mathrm{ZnBr}_{2}(0.100 \mathrm{~g}$, $0.166 \mathrm{mmol})$, thiophenol $(0.034 \mathrm{~mL}, 0.332 \mathrm{mmol})$ and $6 \mathrm{~mL}$ THF and frozen. A separate vial was charged with potassium bis(trimethylsilyl)amide $(0.066 \mathrm{~g}, 0.332 \mathrm{mmol})$ and $4 \mathrm{~mL}$ THF and frozen. Upon thawing, the solution of $\mathrm{KN}\left(\mathrm{SiMe}_{3}\right)_{2}$ was added dropwise to the solution containing $\left({ }^{3-\mathrm{BBN}} \mathrm{NN}^{\mathrm{tBu}}\right) \mathrm{ZnBr}_{2}$ and stirred while warming up to RT for $1 \mathrm{hr}$. The solution was filtered through Celite and dried. The material was washed with $10 \mathrm{~mL}$ diethyl ether and dried to afford white powder $(0.083 \mathrm{~g}, 0.125 \mathrm{mmol}, 76 \%)$ assigned as $\left({ }^{3-B B N} N^{t B u}\right) Z n(S P h)_{2}$. Single, $\mathrm{X}$-ray quality crystals were obtained by diffusing $n$-pentane into a benzene solution of $\left({ }^{3-B B N} \mathrm{NN}^{t B u}\right) \mathrm{Zn}(\mathrm{SPh})_{2}$ at RT. ${ }^{1} \mathrm{H}$ NMR $\left(\mathrm{C}_{6} \mathrm{D}_{6}, 25^{\circ} \mathrm{C}\right) \delta=1.09\left(\mathrm{~s}, 9 \mathrm{H}, \mathrm{C}\left(\mathrm{CH}_{3}\right)_{3}\right), 1.30(\mathrm{t}, \mathrm{J}=7.8,2 \mathrm{H}, \mathrm{B}-$ $\left.\mathrm{CH}_{2}\right), 1.49(\mathrm{~m}, 2 \mathrm{H}, \mathrm{BBN}-\mathrm{CH}), 1.72(\mathrm{~m}, 2 \mathrm{H}, \mathrm{BBN}-\mathrm{CH}), 1.95(\mathrm{~m}, 2 \mathrm{H}, \mathrm{BBN}-\mathrm{CH}), 2.01\left(\mathrm{~m}, 8 \mathrm{H}, \mathrm{BBN}-\mathrm{CH}_{2}\right), 2.59$ (s, $\left.3 \mathrm{H}, \mathrm{CH}_{3}\right), 2.63\left(\mathrm{p}, J=8.4,2 \mathrm{H}, \mathrm{CH}_{2} \mathrm{CH}_{2} \mathrm{CH}_{2}\right), 4.15\left(\mathrm{t}, J=7.7,2 \mathrm{H}, \mathrm{N}-\mathrm{CH}_{2}\right), 6.00(\mathrm{~s}, 1 \mathrm{H}$, pyrazole- $\mathrm{CH}), 6.19(\mathrm{~d}, J=$ 7.7, $1 \mathrm{H}$, pyridine- $\mathrm{CH}), 6.58(\mathrm{~d}, J=7.7,1 \mathrm{H}$, pyridine- $\mathrm{CH}), 6.76(\mathrm{t}, J=7.6,2 \mathrm{H}, p$-phenyl- $\mathrm{CH}), 6.78(\mathrm{t}, J=7.7$, $1 \mathrm{H}, p$-pyridine- $\mathrm{CH}), 6.85(\mathrm{t}, J=7.6,4 \mathrm{H}, m$-phenyl- $\mathrm{CH}), 7.55\left(\mathrm{~d}, J=7.6,4 \mathrm{H}, o\right.$-phenyl-CH). ${ }^{13} \mathrm{C}$ NMR $\left(\mathrm{C}_{6} \mathrm{D}_{6}, 25\right.$

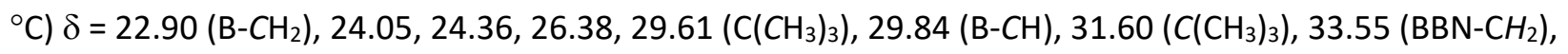
$54.46\left(\mathrm{~N}-\mathrm{CH}_{2}\right), 100.90$ (pyrazole- $\mathrm{CH}$ ), 117.19, 123.17, 124.57, 128.35, 133.77, 139.43, 142.36, 145.90, $146.72,156.25,159.13$. Selected IR data $(\mathrm{KBr}) \mathrm{v}=3065,1610,1578,1474,1371,1244,1088,1024,794$, $737,694 \mathrm{~cm}^{-1}$.

Synthesis of $\left({ }^{4-B B N} N^{t B u}\right) Z n(S P h)_{2}$. A $20 \mathrm{~mL}$ scintillation vial was charged with $\left({ }^{4-B B N} \mathrm{NN}^{t B u}\right) \mathrm{ZnBr}_{2}(0.117 \mathrm{~g}$, $0.190 \mathrm{mmol}$ ) and $6 \mathrm{~mL}$ THF. While stirring, thiophenol $(0.039 \mathrm{~mL}, 0.381 \mathrm{mmol})$ was added and the solution frozen. A separate vial was charged with potassium bis(trimethylsilyl)amide $(0.076 \mathrm{~g}, 0.381 \mathrm{mmol})$ and 2 $\mathrm{mL}$ THF and frozen. Upon thawing, the solution of $\mathrm{KN}\left(\mathrm{SiMe}_{3}\right)_{2}$ was added dropwise to the solution containing ( $\left.{ }^{4-B B N} \mathrm{NN}^{t B u}\right) \mathrm{ZnBr}_{2}$. The reaction was stirred for 20 min while warming up to RT, filtered through Celite, and dried. The resulting material was washed with $10 \mathrm{~mL} n$-pentane then precipitated from minimal diethyl ether with $n$-pentane. The resulting material was dried to afford a white powder $(0.091$ g, $0.135 \mathrm{mmol}, 71 \%)$ assigned as $\left({ }^{4-B B N} \mathrm{NN}^{t B u}\right) \mathrm{Zn}(\mathrm{SPh})_{2}$. Single, $\mathrm{X}$-ray quality crystals were obtained by layering a $\mathrm{C}_{6} \mathrm{H}_{6}$ solution of $\left({ }^{4-B B N} \mathrm{NN}^{\mathrm{tBu}}\right) \mathrm{Zn}(\mathrm{SPh})_{2}$ with hexamethyldisiloxane at $\mathrm{RT}$. ${ }^{1} \mathrm{H} \mathrm{NMR}\left(\mathrm{C}_{6} \mathrm{D}_{6}, 25{ }^{\circ} \mathrm{C}\right) \delta=$ $1.02\left(\mathrm{~s}, 9 \mathrm{H}, \mathrm{C}\left(\mathrm{CH}_{3}\right)_{3}\right), 1.22\left(\mathrm{~m}, 2 \mathrm{H}, \mathrm{B}-\mathrm{CH}_{2}\right), 1.51(\mathrm{~m}, 2 \mathrm{H}, \mathrm{B}-\mathrm{CH}), 1.59\left(\mathrm{~m}, 2 \mathrm{H}, \mathrm{CH}_{2} \mathrm{CH}_{2} \mathrm{~B}\right), 1.71(\mathrm{~m}, 2 \mathrm{H}, \mathrm{BBN}-$ $\mathrm{CH}), 1.77(\mathrm{~m}, 2 \mathrm{H}, \mathrm{BBN}-\mathrm{CH}), 1.86\left(\mathrm{~m}, 8 \mathrm{H}, \mathrm{BBN}-\mathrm{CH}_{2}\right), 2.43\left(\mathrm{~m}, 2 \mathrm{H}, \mathrm{NCH}_{2} \mathrm{CH}_{2}\right), 2.72\left(\mathrm{~s}, 3 \mathrm{H}, \mathrm{CH}_{3}\right), 4.27(\mathrm{t}, \mathrm{J}=8.4$, $\left.2 \mathrm{H}, \mathrm{NCH}_{2}\right), 5.90(\mathrm{~s}, 1 \mathrm{H}$, pyrazole- $\mathrm{CH}), 6.24(\mathrm{~d}, J=7.8,1 \mathrm{H}$, pyridine- $\mathrm{CH}), 6.56(\mathrm{~d}, J=7.9,1 \mathrm{H}$, pyridine- $\mathrm{CH})$, 6.79 (overlapping: $2 \mathrm{H}, p$-phenyl- $\mathrm{CH} ; 1 \mathrm{H}, p$-pyridine- $\mathrm{CH}), 6.88(\mathrm{t}, J=7.6,4 \mathrm{H}, m$-phenyl- $\mathrm{CH}), 7.64(\mathrm{~d}, J=7.6$, $4 \mathrm{H}$, o-phenyl-CH). ${ }^{13} \mathrm{C}$ NMR $\left(\mathrm{C}_{6} \mathrm{D}_{6}, 25{ }^{\circ} \mathrm{C}\right) \delta=22.37,23.67,24.20,27.87\left(\mathrm{~B}-\mathrm{CH}_{2}\right), 29.67\left(\mathrm{C}\left(\mathrm{CH}_{3}\right)_{3}\right), 31.32$,

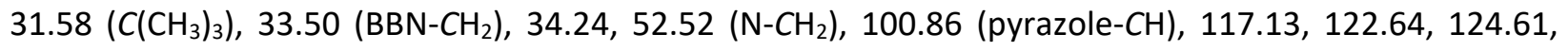
$133.70,139.28,143.72,145.96,146.87,155.41,159.48 .{ }^{11} \mathrm{~B} \mathrm{NMR}\left(\mathrm{C}_{6} \mathrm{D}_{6}, 25{ }^{\circ} \mathrm{C}\right) \delta=87.06$. Selected IR data $(\mathrm{KBr}) \mathrm{v}=3062,1610,1577,1367,1085,1024,795,739,695 \mathrm{~cm}^{-1}$. 


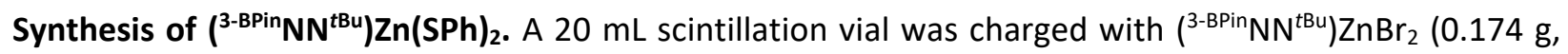
$0.286 \mathrm{mmol})$ and $6 \mathrm{~mL}$ THF. While stirring, thiophenol $(0.059 \mathrm{~mL}, 0.577 \mathrm{mmol})$ was added and the solution frozen. A separate vial was charged with potassium bis(trimethylsilyl)amide $(0.114 \mathrm{~g}, 0.571 \mathrm{mmol})$ and 2 $\mathrm{mL}$ THF and frozen. Upon thawing, the solution of $\mathrm{KN}\left(\mathrm{SiMe}_{3}\right)_{2}$ was added dropwise to the solution containing ( $\left.{ }^{3-B P i n} \mathrm{NN}^{\mathrm{tBu}}\right) \mathrm{ZnBr}_{2}$. The reaction was stirred for 20 min while warming up to $\mathrm{RT}$, filtered through Celite, and dried. The resulting material was washed with $10 \mathrm{~mL} n$-pentane then precipitated from minimal diethyl ether with $n$-pentane. The resulting material was dried to afford a white powder $(0.167$ g, $0.250 \mathrm{mmol}, 88 \%)$ assigned as $\left({ }^{3-B P i n} \mathrm{NN}^{\mathrm{tBu}}\right) \mathrm{Zn}(\mathrm{SPh})_{2}$. Single, $\mathrm{X}$-ray quality crystals were obtained by layering a $\mathrm{C}_{6} \mathrm{H}_{6}$ solution of $\left({ }^{3-B P i n} \mathrm{NN}^{t \mathrm{Bu}}\right) \mathrm{Zn}(\mathrm{SPh})_{2}$ with hexamethyldisiloxane at RT. High-Res MS of $\mathrm{C}_{34} \mathrm{H}_{44} \mathrm{~N}_{3} \mathrm{O}_{2} \mathrm{~B}_{1} \mathrm{~S}_{2} \mathrm{Zn}_{1}$ - SPh: Calc. 556.2146; Found 556.2137. ${ }^{1} \mathrm{H}$ NMR $\left(\mathrm{C}_{6} \mathrm{D}_{6}, 25{ }^{\circ} \mathrm{C}\right) \delta=1.02\left(\mathrm{~m}, 2 \mathrm{H}, \mathrm{B}-\mathrm{CH}_{2}\right)$, $1.05\left(\mathrm{~s}, 9 \mathrm{H}, \mathrm{C}\left(\mathrm{CH}_{3}\right)_{3}\right), 1.08\left(\mathrm{~s}, 12 \mathrm{H}, \mathrm{BPin}-\mathrm{CH}_{3}\right), 2.56\left(\mathrm{p}, \mathrm{J}=8.6,2 \mathrm{H}, \mathrm{CH}_{2} \mathrm{CH}_{2} \mathrm{CH}_{2}\right), 2.73\left(\mathrm{~s}, 3 \mathrm{H}, \mathrm{CH}_{3}\right), 4.34(\mathrm{t}, J=$ 8.6, $\left.2 \mathrm{H}, \mathrm{NCH}_{2}\right), 5.90(\mathrm{~s}, 1 \mathrm{H}$, pyrazole- $\mathrm{CH}), 6.25(\mathrm{~d}, J=7.7,1 \mathrm{H}$, pyridine- $\mathrm{CH}), 6.57(\mathrm{~d}, J=7.7,1 \mathrm{H}$, pyridine$\mathrm{CH}), 6.79(\mathrm{t}, J=7.5,2 \mathrm{H}, p$-phenyl- $\mathrm{CH}), 6.81(\mathrm{t}, J=7.7,1 \mathrm{H}, p$-pyridine- $\mathrm{CH}) 6.88(\mathrm{t}, J=7.5,4 \mathrm{H}, m$-phenyl- $\mathrm{CH})$, $7.64\left(\mathrm{~d}, J=7.6,4 \mathrm{H}, o\right.$-phenyl-CH). ${ }^{13} \mathrm{C}$ NMR $\left(\mathrm{C}_{6} \mathrm{D}_{6}, 25^{\circ} \mathrm{C}\right) \delta=8.35\left(\mathrm{~B}-\mathrm{CH}_{2}\right), 24.18,25.05$ (BPin- $\left.\mathrm{CH}_{3}\right), 25.20$,

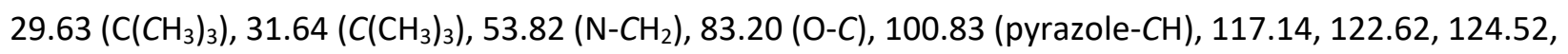
$128.35,133.85,139.26,143.80,145.89,146.94,155.47,159.37 .{ }^{11} \mathrm{~B} N M R\left(\mathrm{C}_{6} \mathrm{D}_{6}, 25^{\circ} \mathrm{C}\right) \delta=34.08$. Selected IR data $(\mathrm{KBr}) \mathrm{v}=3065,2975,1610,1578,1473,1434,1383,1316,1186,797,738,694 \mathrm{~cm}^{-1}$.

Attempted Synthesis of $\left({ }^{2-B B N} N^{t B u}\right) Z n(O P h)_{2}$. A $20 \mathrm{~mL}$ scintillation vial was charged with $\left({ }^{2-B B N} \mathrm{NN}^{t B u}\right) \mathrm{ZnBr}_{2}$ (0.075 g, $0.124 \mathrm{mmol})$, phenol $(0.212 \mathrm{M}$ in THF, $1.175 \mathrm{~mL}, 0.249 \mathrm{mmol}$ ) and $6 \mathrm{~mL}$ THF and frozen. A separate vial was charged with potassium bis(trimethylsilyl)amide $(0.050 \mathrm{~g}, 0.249 \mathrm{mmol})$ and $4 \mathrm{~mL}$ THF and frozen. Upon thawing, the solution of $\mathrm{KN}\left(\mathrm{SiMe}_{3}\right)_{2}$ was added dropwise to the solution containing ${ }^{3-}$ $\left.{ }^{\mathrm{BBN}} \mathrm{NN}^{\mathrm{tBu}}\right) \mathrm{ZnBr}_{2}$ and stirred while warming up to room temperature for $30 \mathrm{~min}$. The solution was filtered through Celite and dried. The material was washed with $2 \times 10 \mathrm{~mL} n$-pentane and dried to afford a white powder. ${ }^{1} \mathrm{H}$ NMR analysis of this white powder revealed an intractable mixture of species, see Figure S26. 


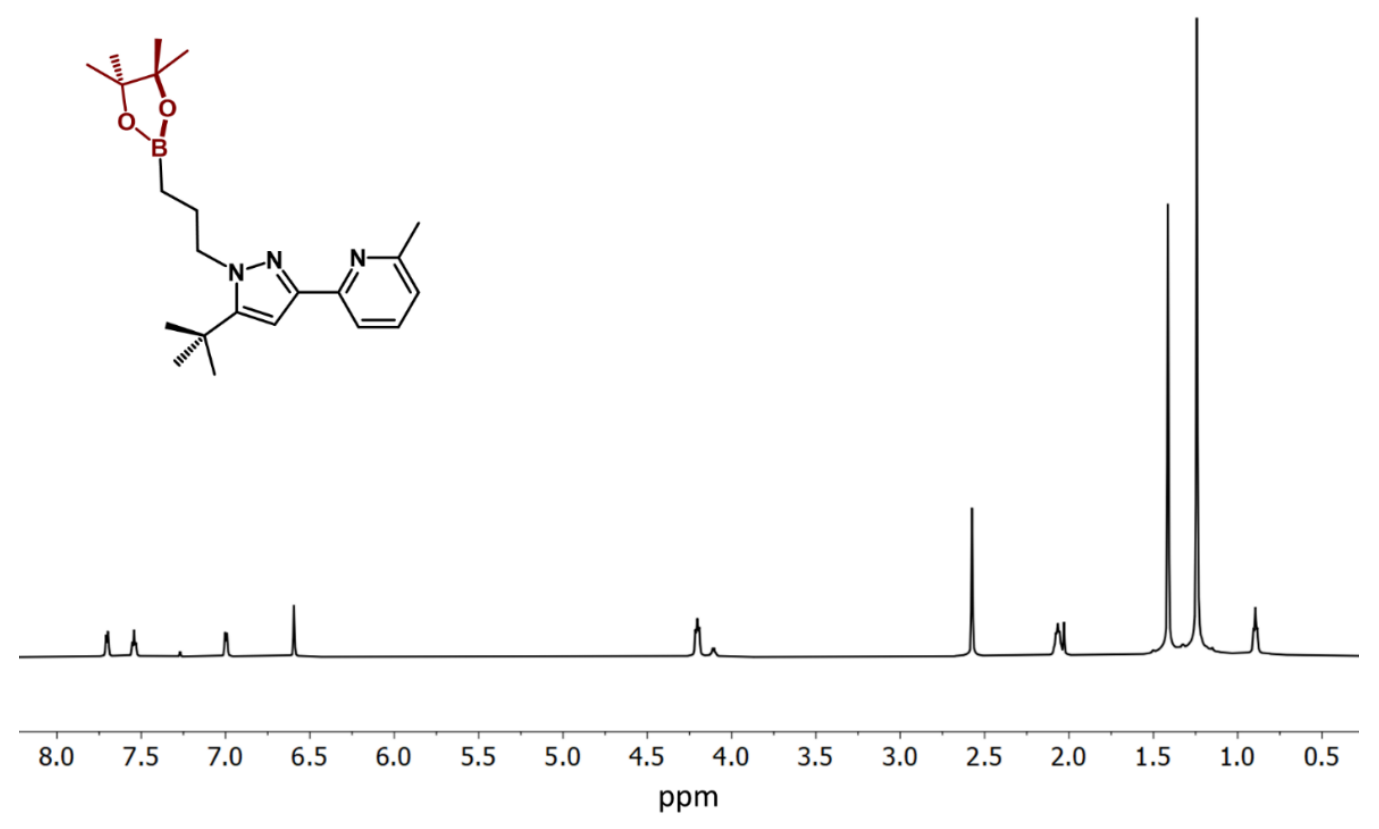

Figure $\mathrm{S} 1{ }^{1} \mathrm{H}$ NMR spectrum $\left(\mathrm{CDCl}_{3}, 25{ }^{\circ} \mathrm{C}\right)$ of 2-(5-(tert-butyl)-1-(3-(4,4,5,5-tetramethyl-1,3,2dioxaborolan-2-yl)propyl)-1H-pyrazol-3-yl)-6-methylpyridine ( $\left.{ }^{3-B P i n} \mathrm{NN}^{\mathrm{tBu}}\right)$.
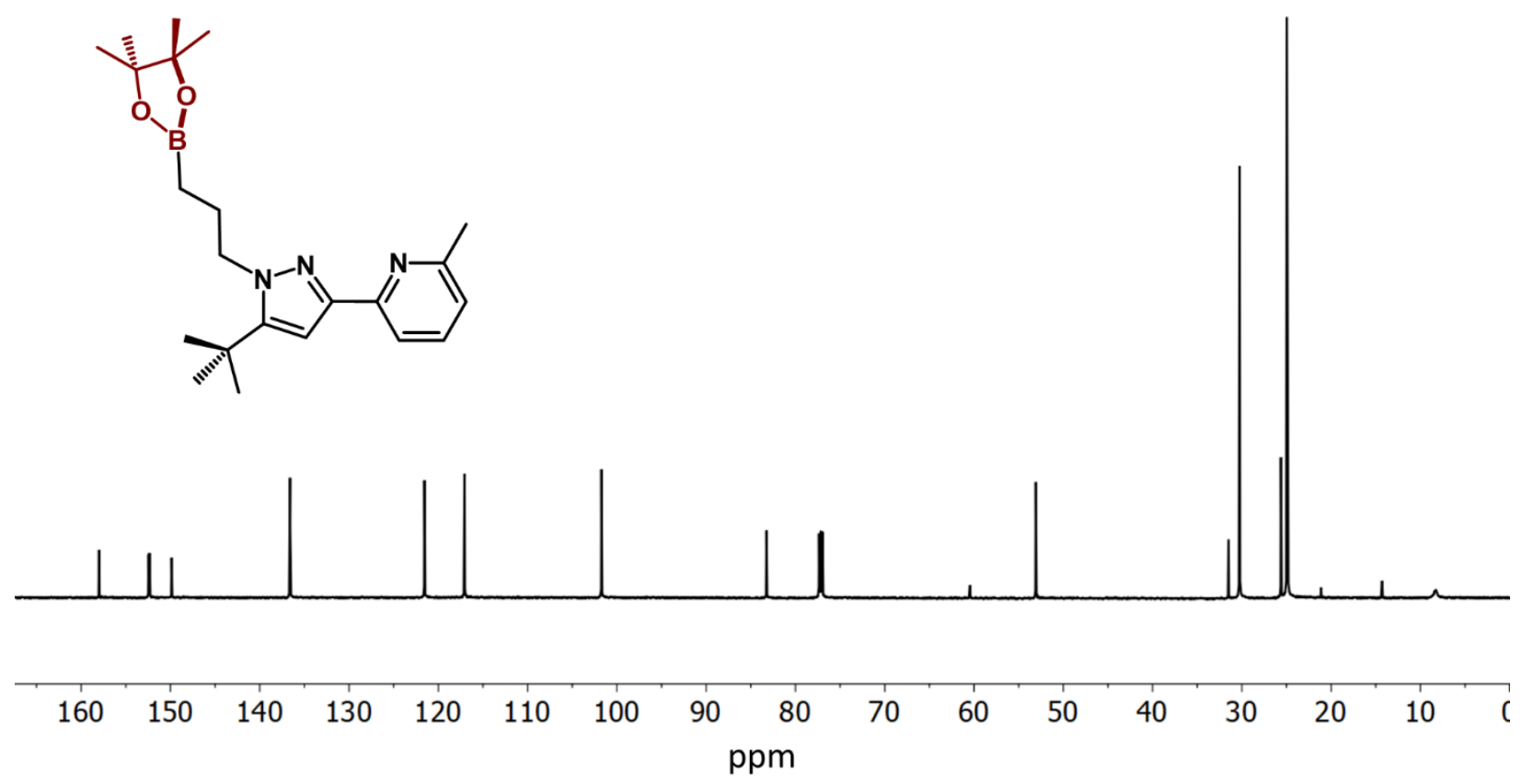

Figure S2 ${ }^{13} \mathrm{C}$ NMR spectrum $\left(\mathrm{CDCl}_{3}, 25{ }^{\circ} \mathrm{C}\right)$ of 2-(5-(tert-butyl)-1-(3-(4,4,5,5-tetramethyl-1,3,2dioxaborolan-2-yl)propyl)-1H-pyrazol-3-yl)-6-methylpyridine ( $\left.{ }^{3-B P i n} \mathrm{NN}^{\mathrm{tBu}}\right)$. 


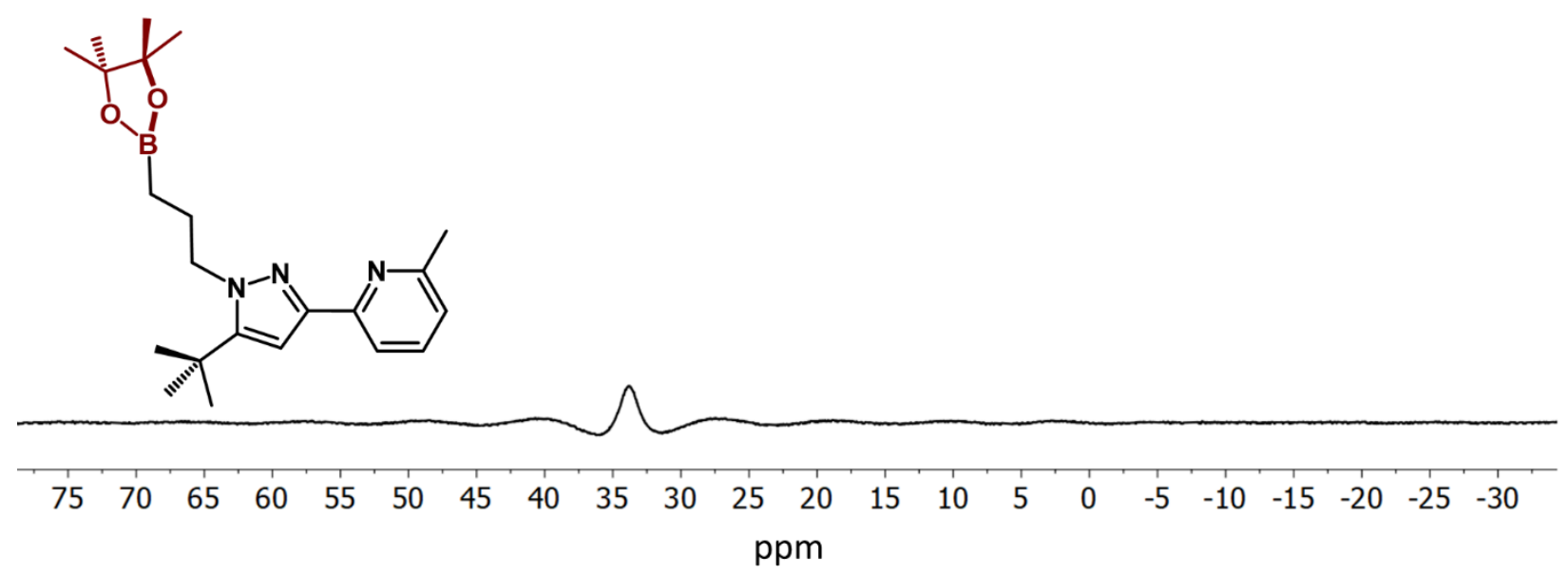

Figure S3 ${ }^{11} \mathrm{~B}$ NMR spectrum $\left(\mathrm{CDCl}_{3}, 25^{\circ} \mathrm{C}\right)$ of 2-(5-(tert-butyl)-1-(3-(4,4,5,5-tetramethyl-1,3,2dioxaborolan-2-yl)propyl)-1H-pyrazol-3-yl)-6-methylpyridine ( $\left.{ }^{3-B P i n} \mathrm{NN}^{t \mathrm{Bu}}\right)$.

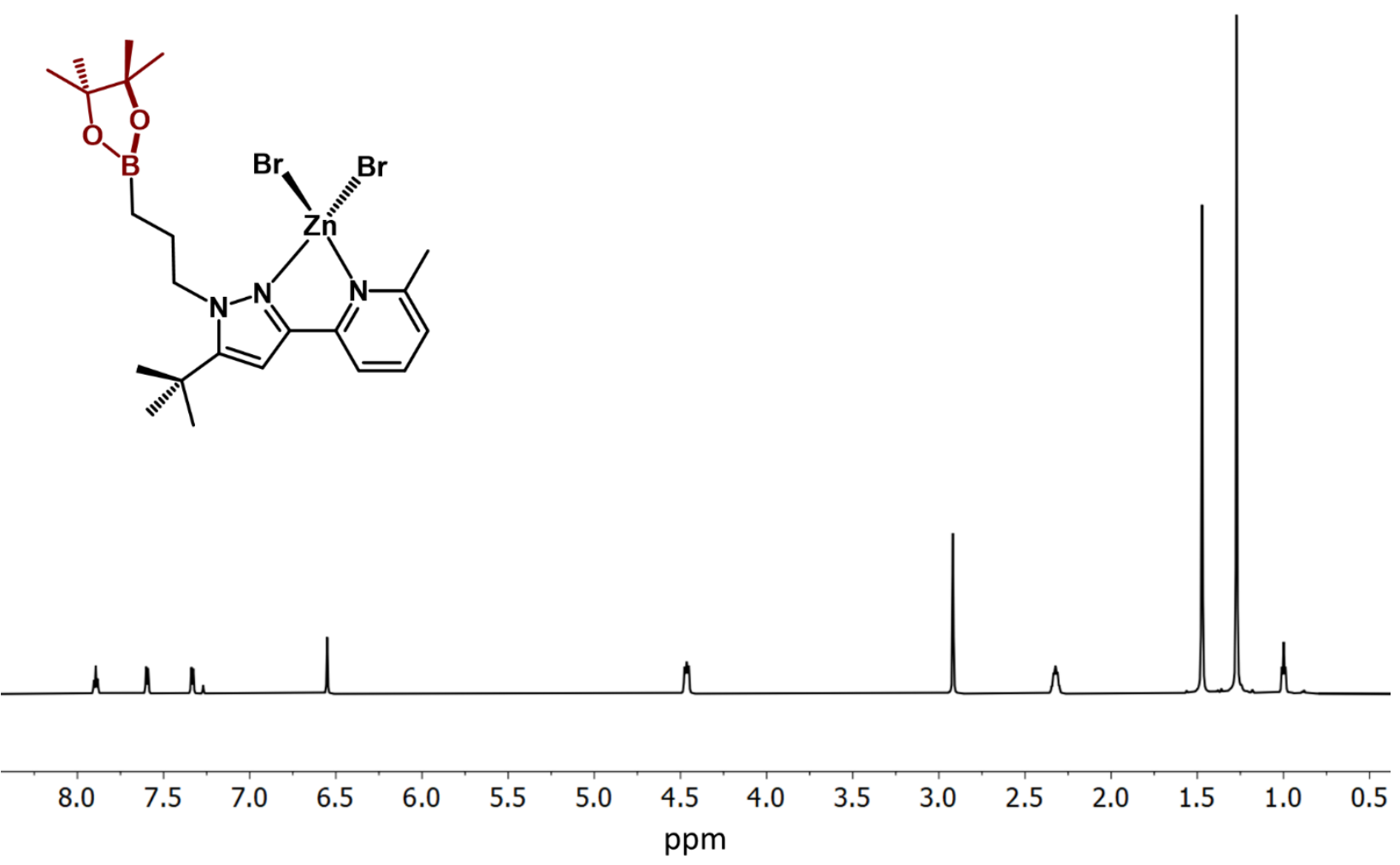

Figure S4 ${ }^{1} \mathrm{H}$ NMR spectrum $\left(\mathrm{CDCl}_{3}, 25^{\circ} \mathrm{C}\right)$ of $\left({ }^{3-\mathrm{BPin}} \mathrm{NN}^{\mathrm{tBu}}\right) \mathrm{ZnBr}_{2}$. 


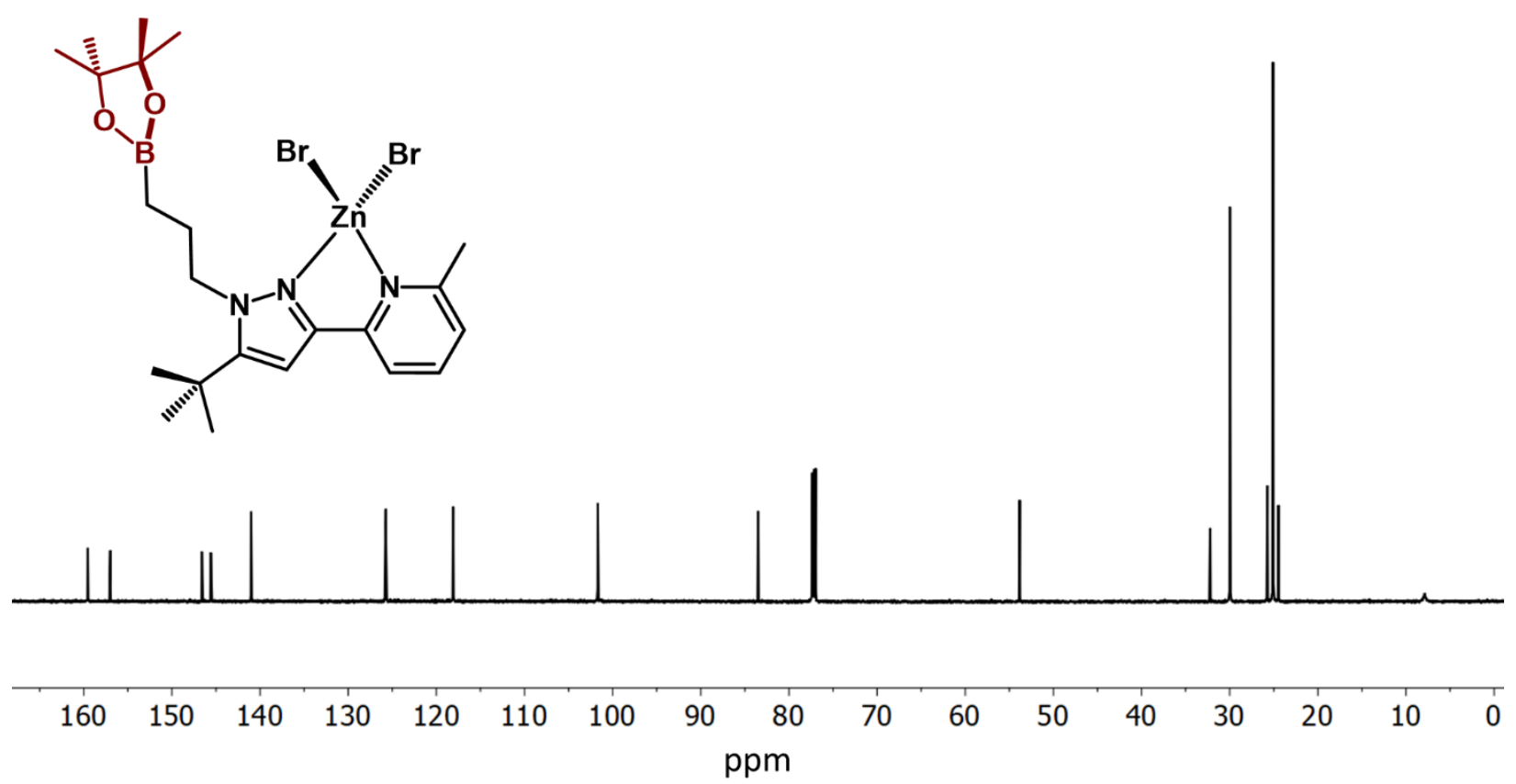

Figure $\mathrm{S} 5{ }^{13} \mathrm{C}$ NMR spectrum $\left(\mathrm{CDCl}_{3}, 25^{\circ} \mathrm{C}\right)$ of $\left({ }^{3-B P i n} \mathrm{NN}^{t \mathrm{Bu}}\right) \mathrm{ZnBr}_{2}$.

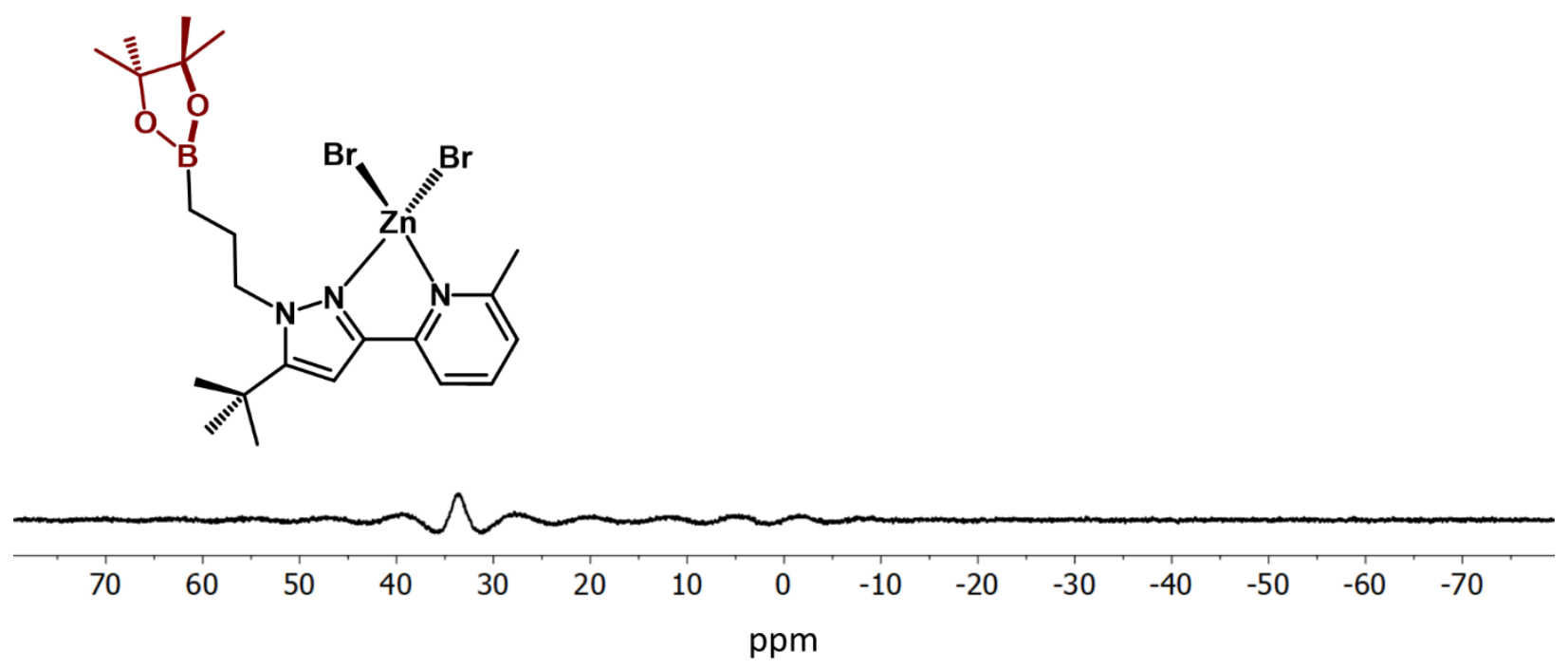

Figure $56{ }^{11} \mathrm{~B}$ NMR spectrum $\left(\mathrm{CDCl}_{3}, 25^{\circ} \mathrm{C}\right)$ of $\left({ }^{3-B P i n} \mathrm{NN}^{\mathrm{tBu}}\right) \mathrm{ZnBr}_{2}$. 


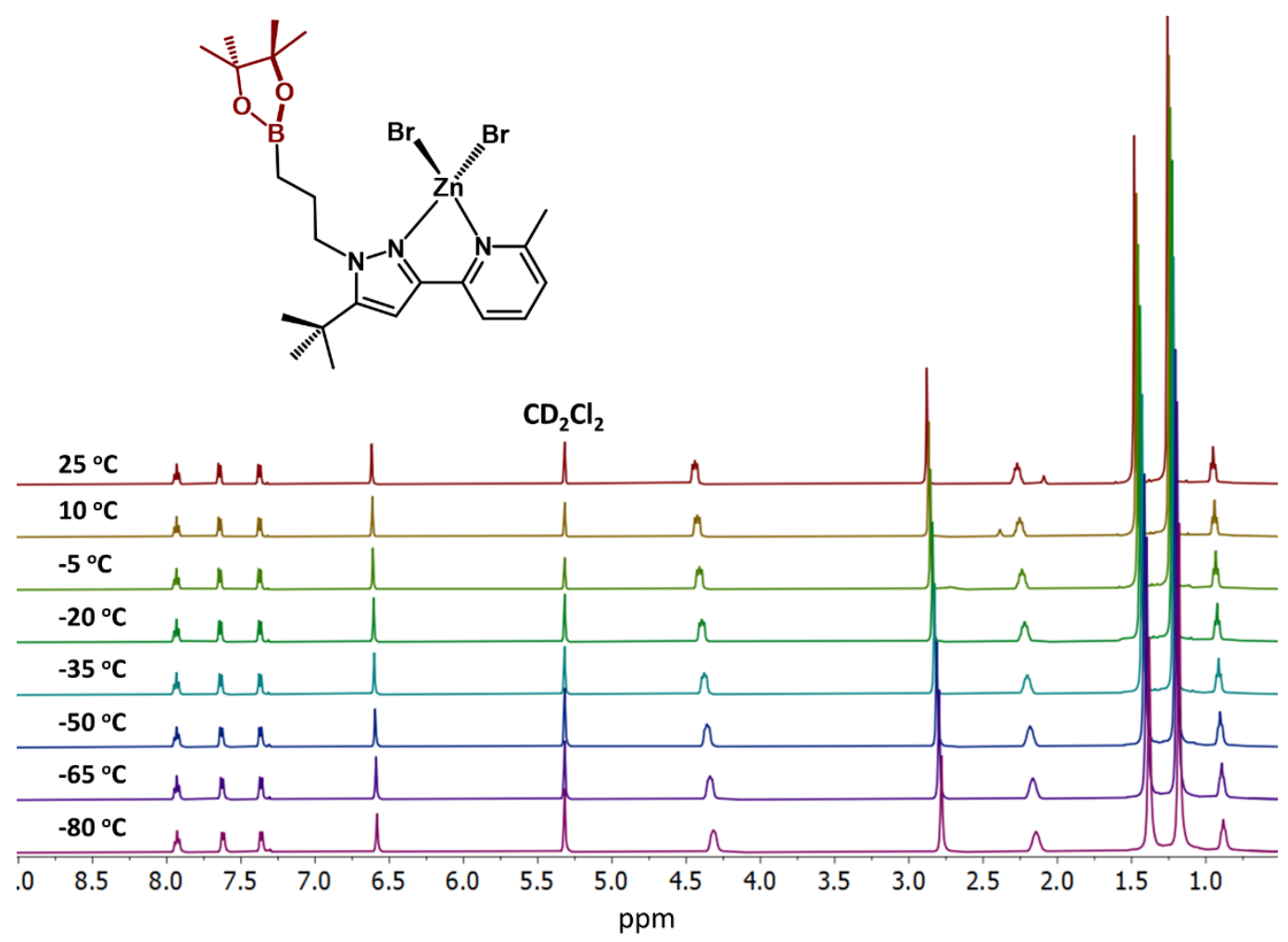

Figure S7 Variable temperature ${ }^{1} \mathrm{H}$ NMR spectra $\left(\mathrm{CD}_{2} \mathrm{Cl}_{2}\right)$ of $\left({ }^{3-B P i n} \mathrm{NN}^{\mathrm{BBu}}\right) \mathrm{ZnBr}_{2}$.
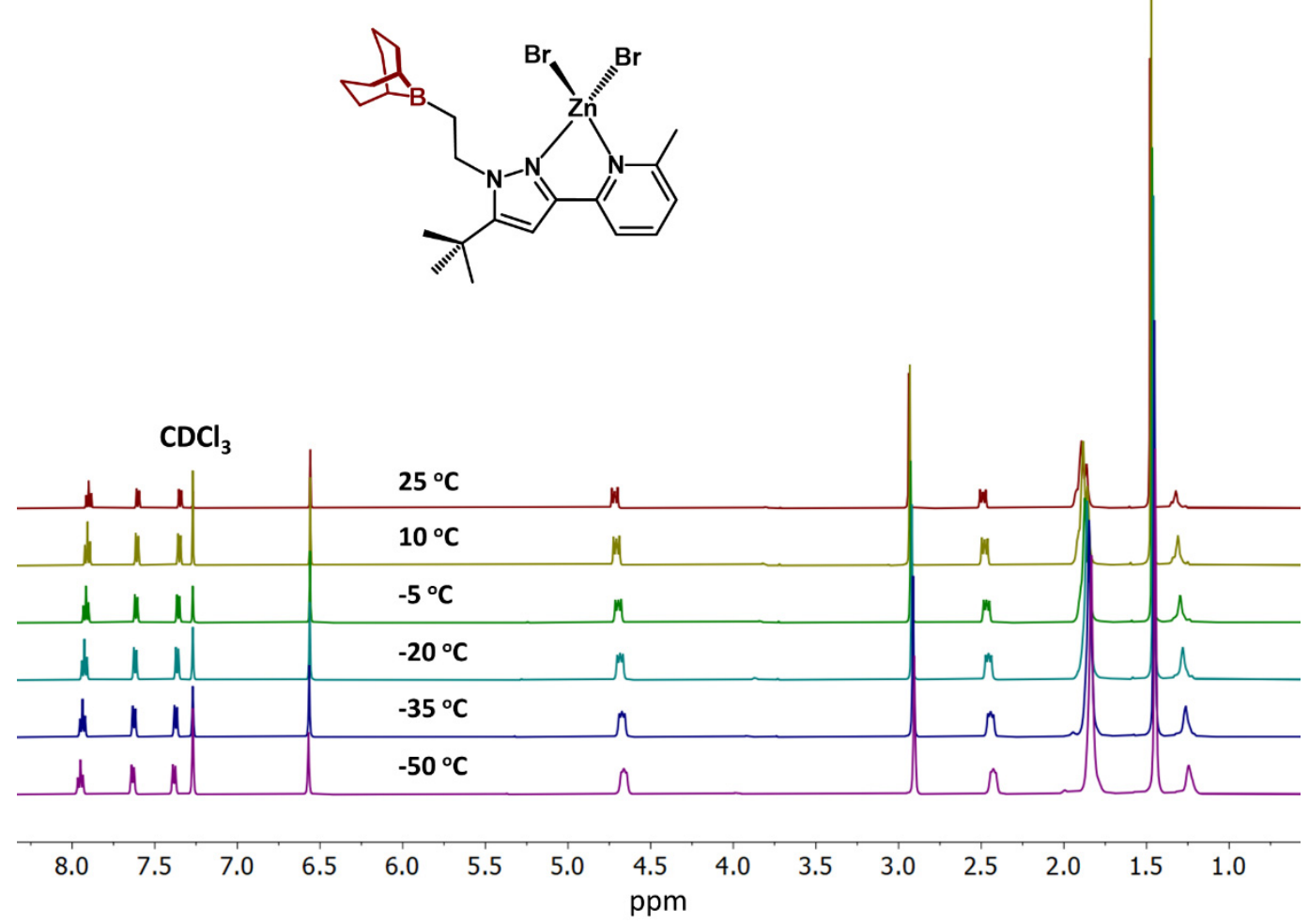

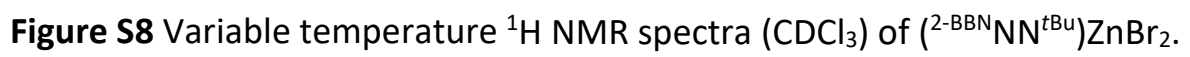




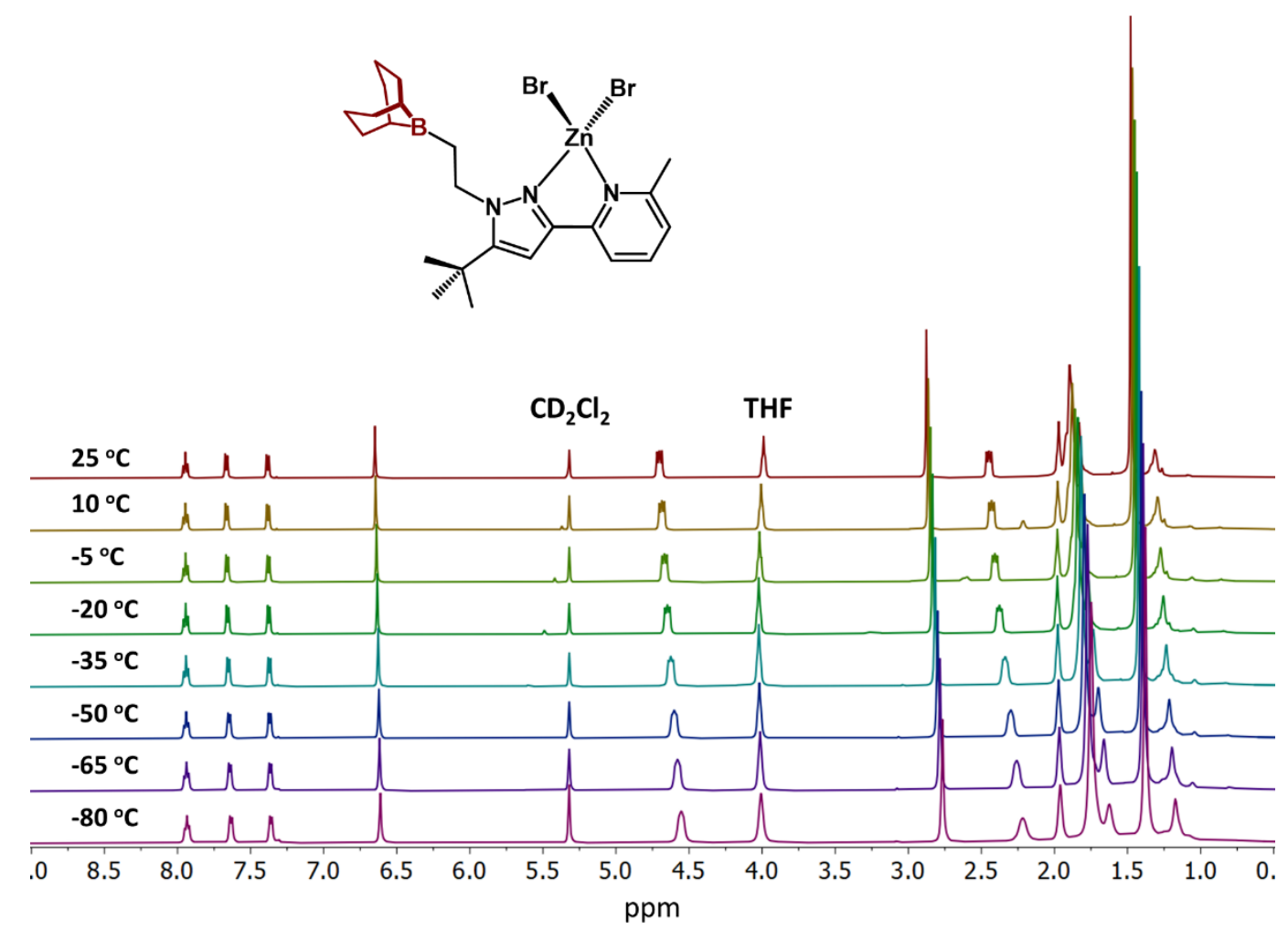

Figure S9 Variable temperature ${ }^{1} \mathrm{H}$ NMR spectra $\left(\mathrm{CD}_{2} \mathrm{Cl}_{2}\right)$ of $\left({ }^{2-\mathrm{BBN}} \mathrm{NN}^{t \mathrm{Bu}}\right) \mathrm{ZnBr}_{2}$.
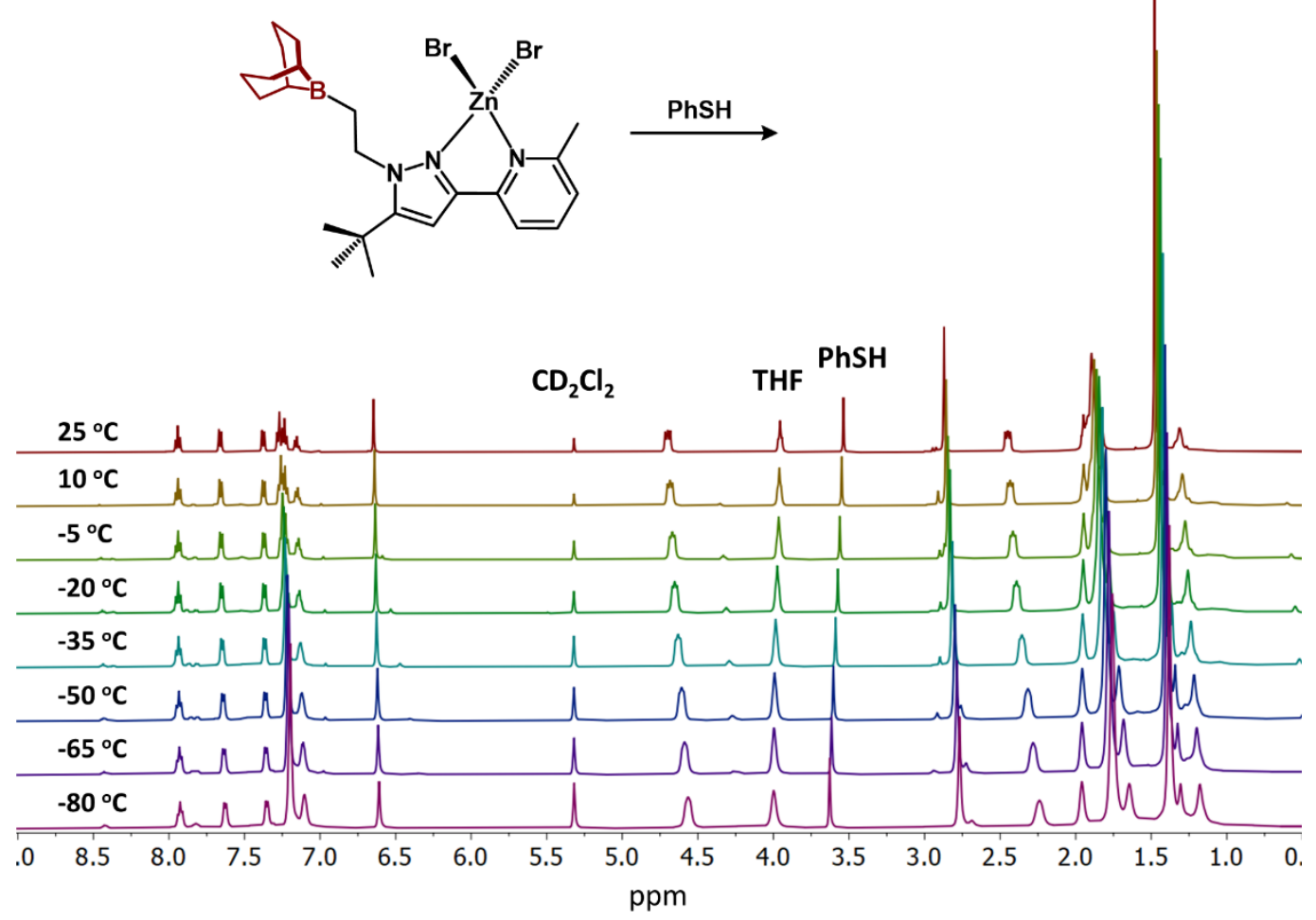

Figure S10 Variable temperature ${ }^{1} \mathrm{H}$ NMR spectra $\left(\mathrm{CD}_{2} \mathrm{Cl}_{2}\right)$ of $\left({ }^{2-B B N} \mathrm{NN}^{t B u}\right) \mathrm{ZnBr}_{2}$ with one equiv thiophenol. 


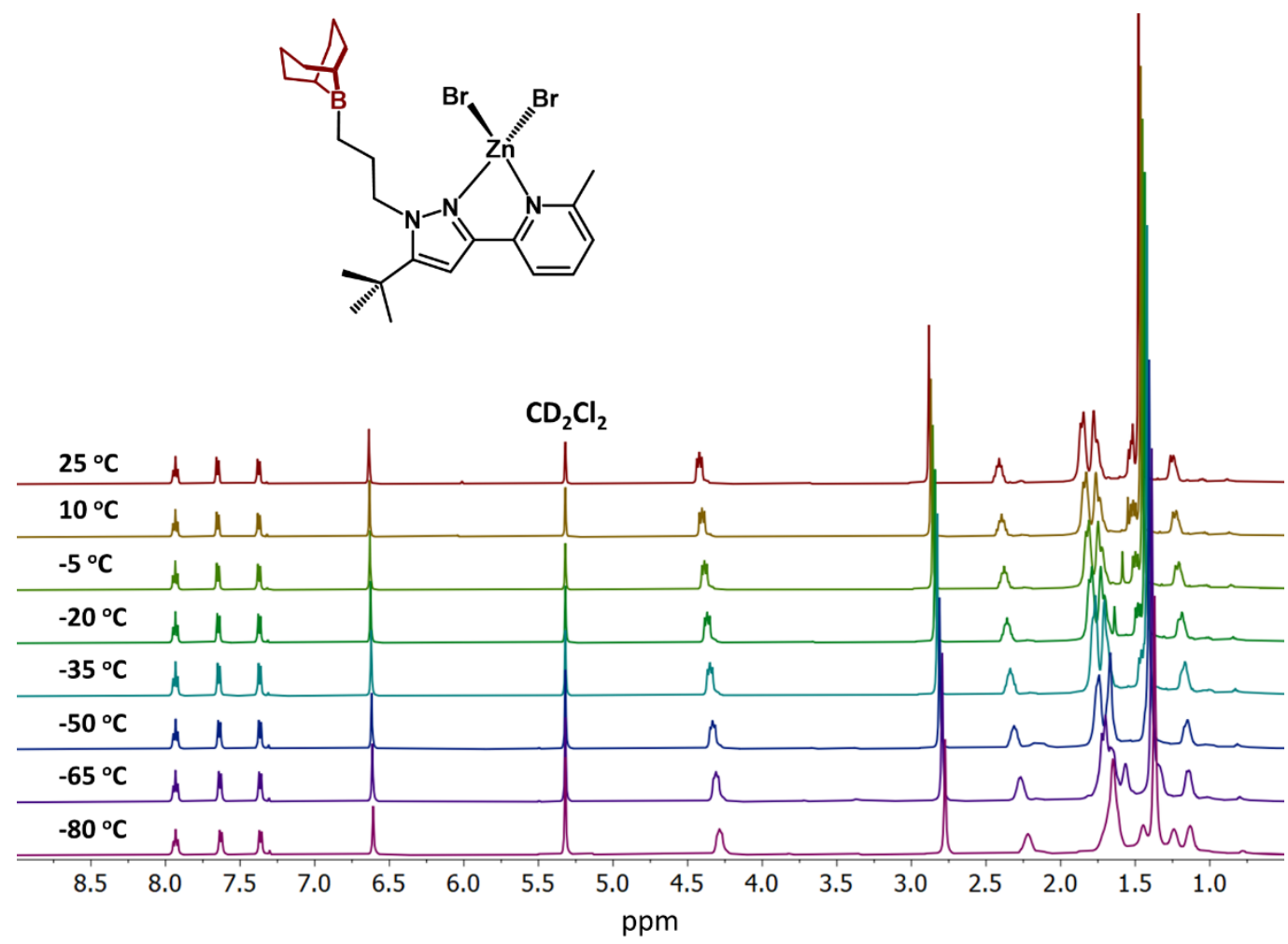

Figure S11 Variable temperature ${ }^{1} \mathrm{H}$ NMR spectra $\left(\mathrm{CD}_{2} \mathrm{Cl}_{2}\right)$ of $\left({ }^{3-B B N} \mathrm{NN}^{t \mathrm{Bu}}\right) \mathrm{ZnBr}_{2}$.

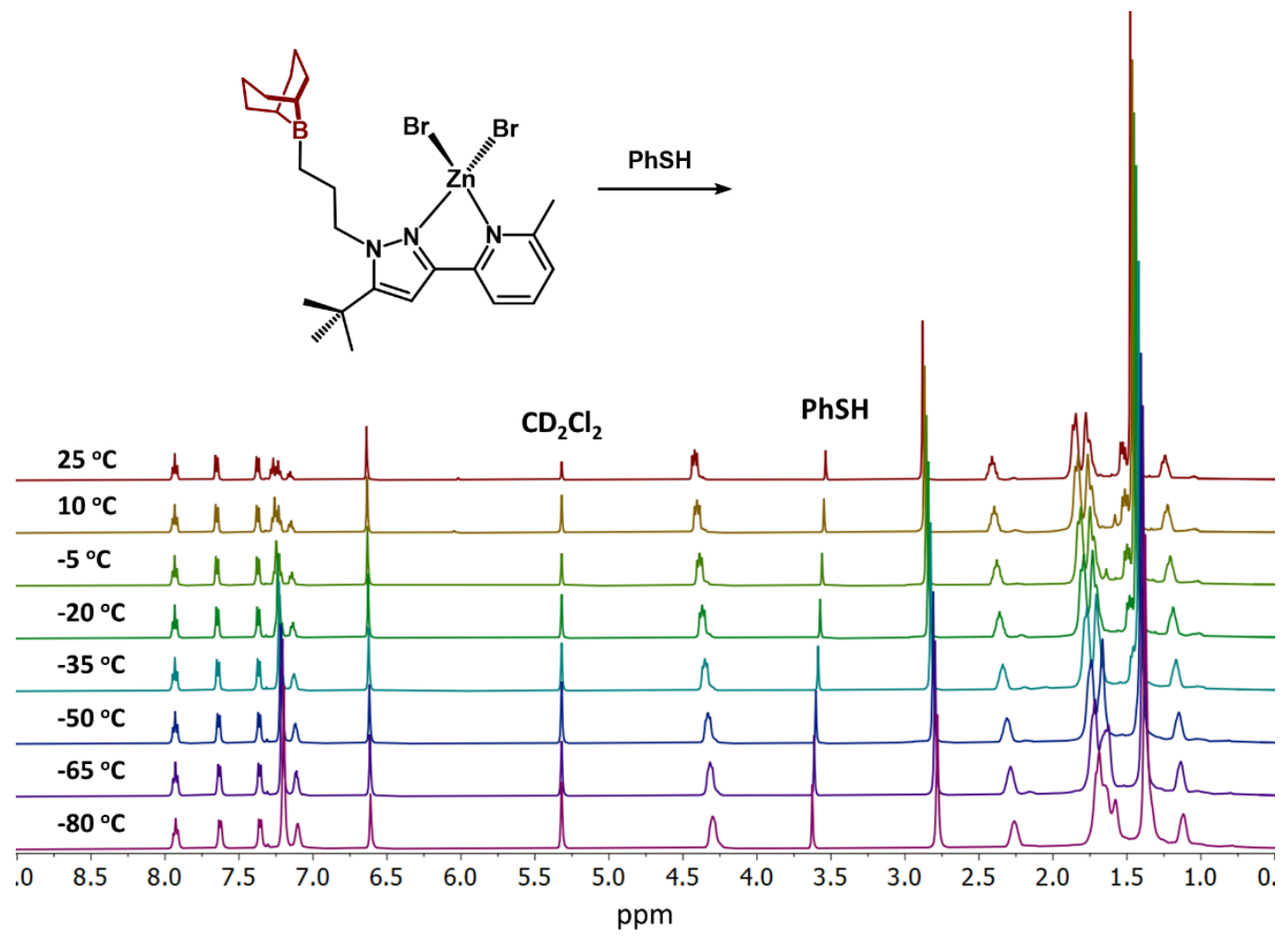

Figure S12 Variable temperature ${ }^{1} \mathrm{H}$ NMR spectra $\left(\mathrm{CD}_{2} \mathrm{Cl}_{2}\right)$ of $\left({ }^{3-\mathrm{BBN}} \mathrm{NN}^{\mathrm{BBu}}\right) \mathrm{ZnBr}_{2}$ with one equiv thiophenol. 


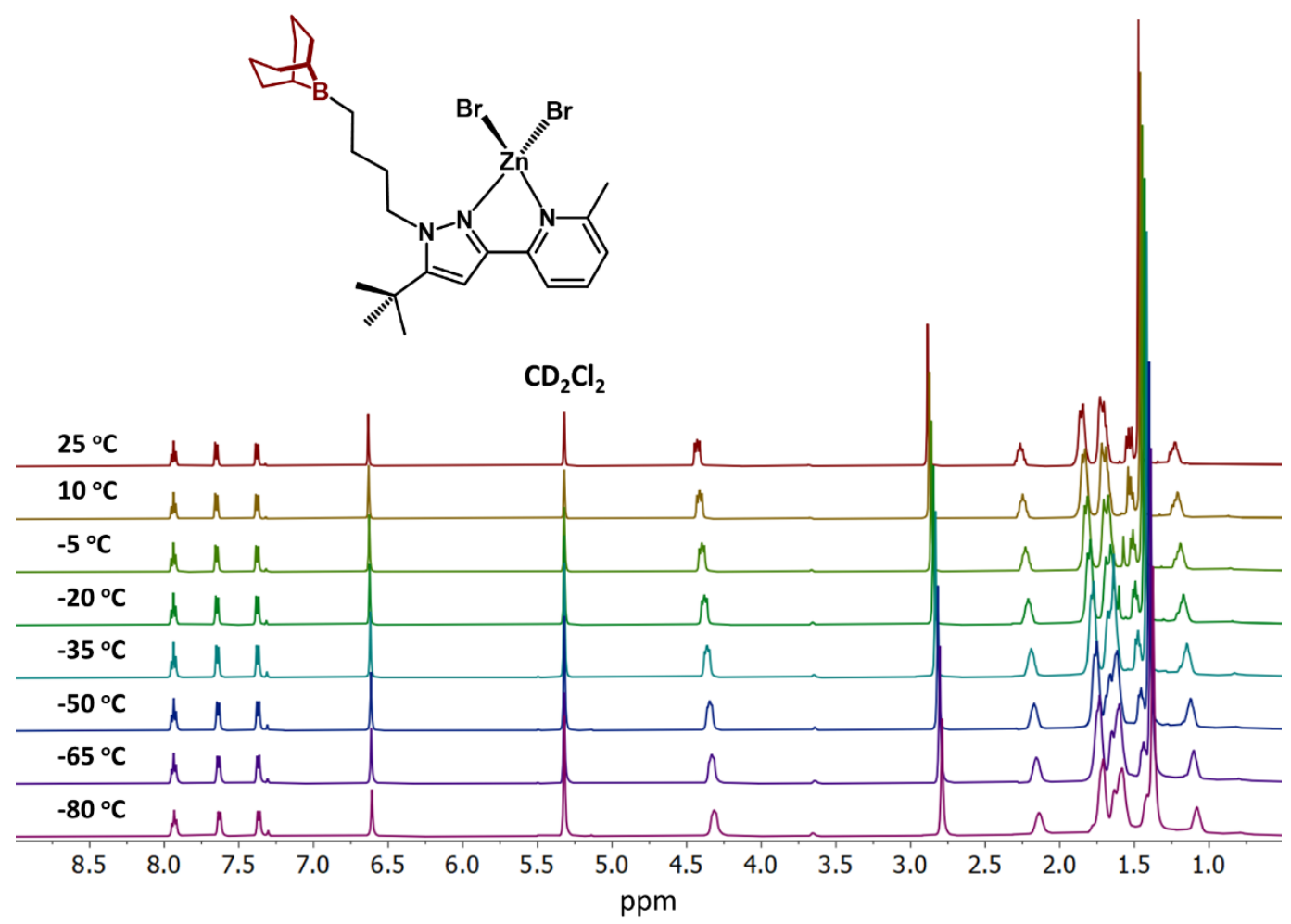

Figure S13 Variable temperature ${ }^{1} \mathrm{H}$ NMR spectra $\left(\mathrm{CD}_{2} \mathrm{Cl}_{2}\right)$ of $\left({ }^{4-B B N} \mathrm{NN}^{t \mathrm{Bu}}\right) \mathrm{ZnBr}_{2}$.
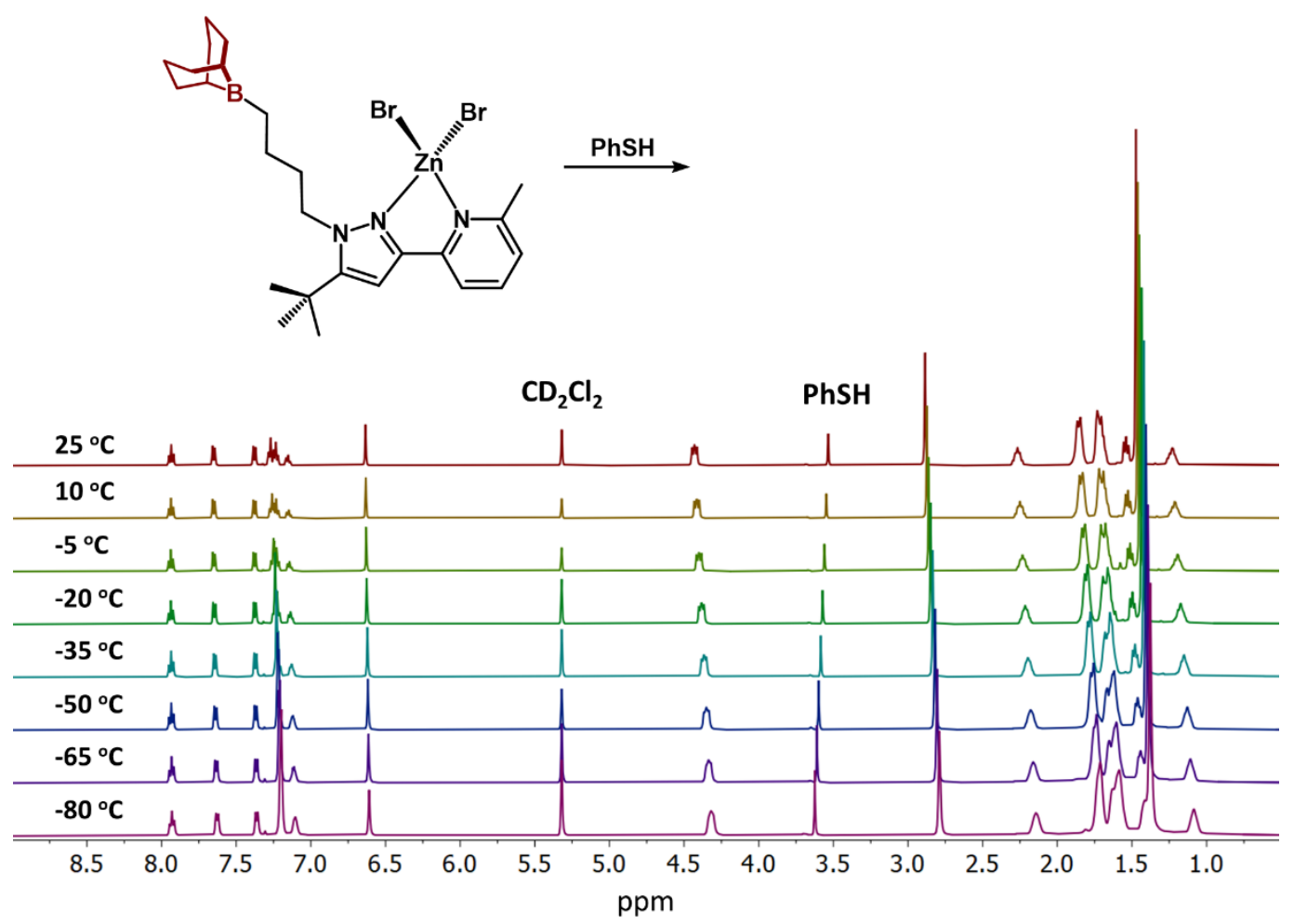

Figure S14 Variable temperature ${ }^{1} \mathrm{H}$ NMR spectra $\left(\mathrm{CD}_{2} \mathrm{Cl}_{2}\right)$ of $\left({ }^{4-B B N} \mathrm{NN}^{\mathrm{BBu}}\right) \mathrm{ZnBr}_{2}$ with one equiv thiophenol. 


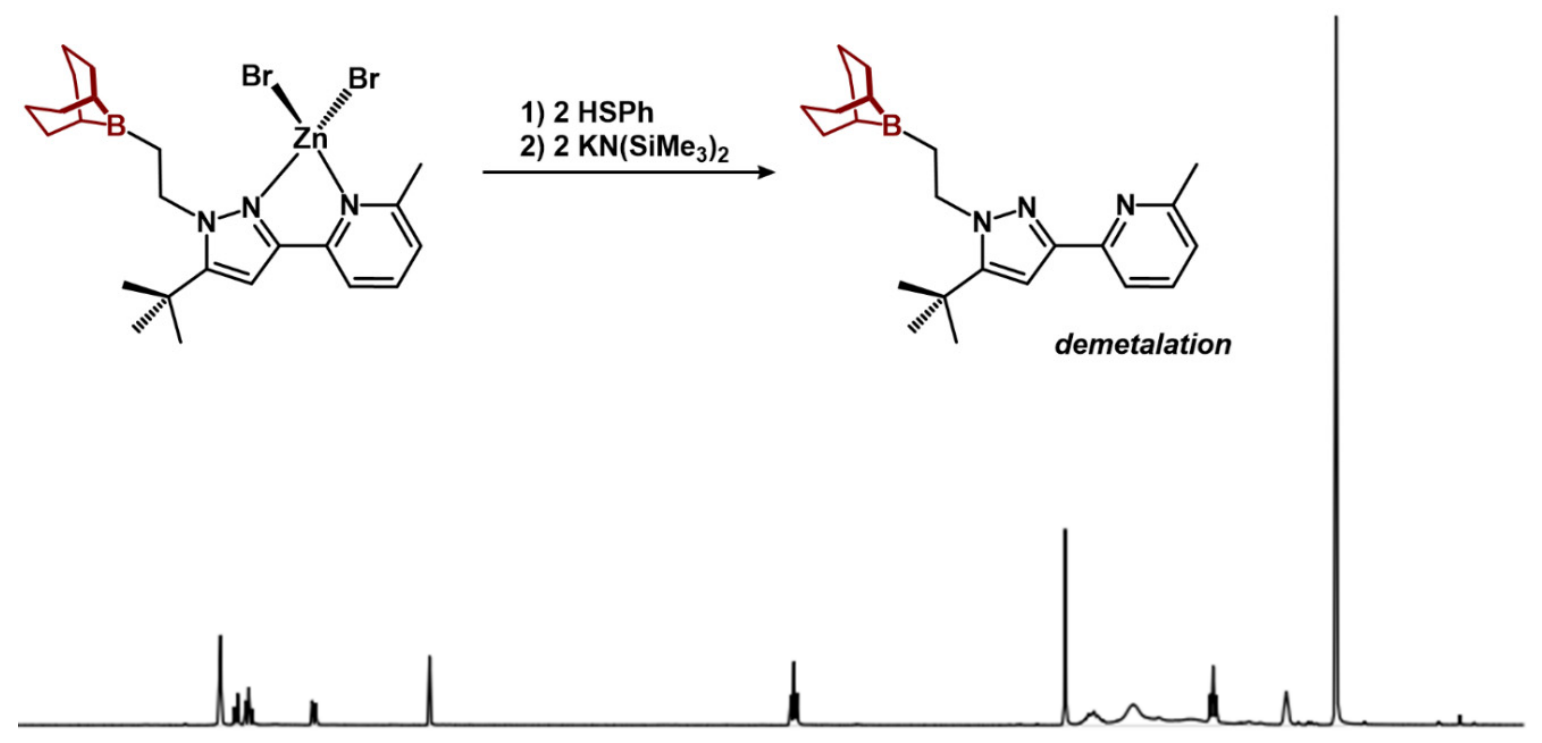

\section{$\begin{array}{llllllllllllllllllll}8.0 & 7.5 & 7.0 & 6.5 & 6.0 & 5.5 & 5.0 & 4.5 & \underset{\mathrm{ppm}}{4.0} & 3.5 & 3.0 & 2.5 & 2.0 & 1.5 & 1.0 & 0.5 & 0.0\end{array}$}

Figure $\mathrm{S} 15{ }^{1} \mathrm{H}$ NMR spectrum $\left(\mathrm{C}_{6} \mathrm{D}_{6}, 25^{\circ} \mathrm{C}\right)$ of attempted synthesis of $\left({ }^{2-B B N} \mathrm{NN}{ }^{t B u}\right) \mathrm{Zn}(\mathrm{SPh})_{2}$. The isolated product is the demetalated ligand, ${ }^{2-B B N} \mathrm{NN}^{t B u}$.

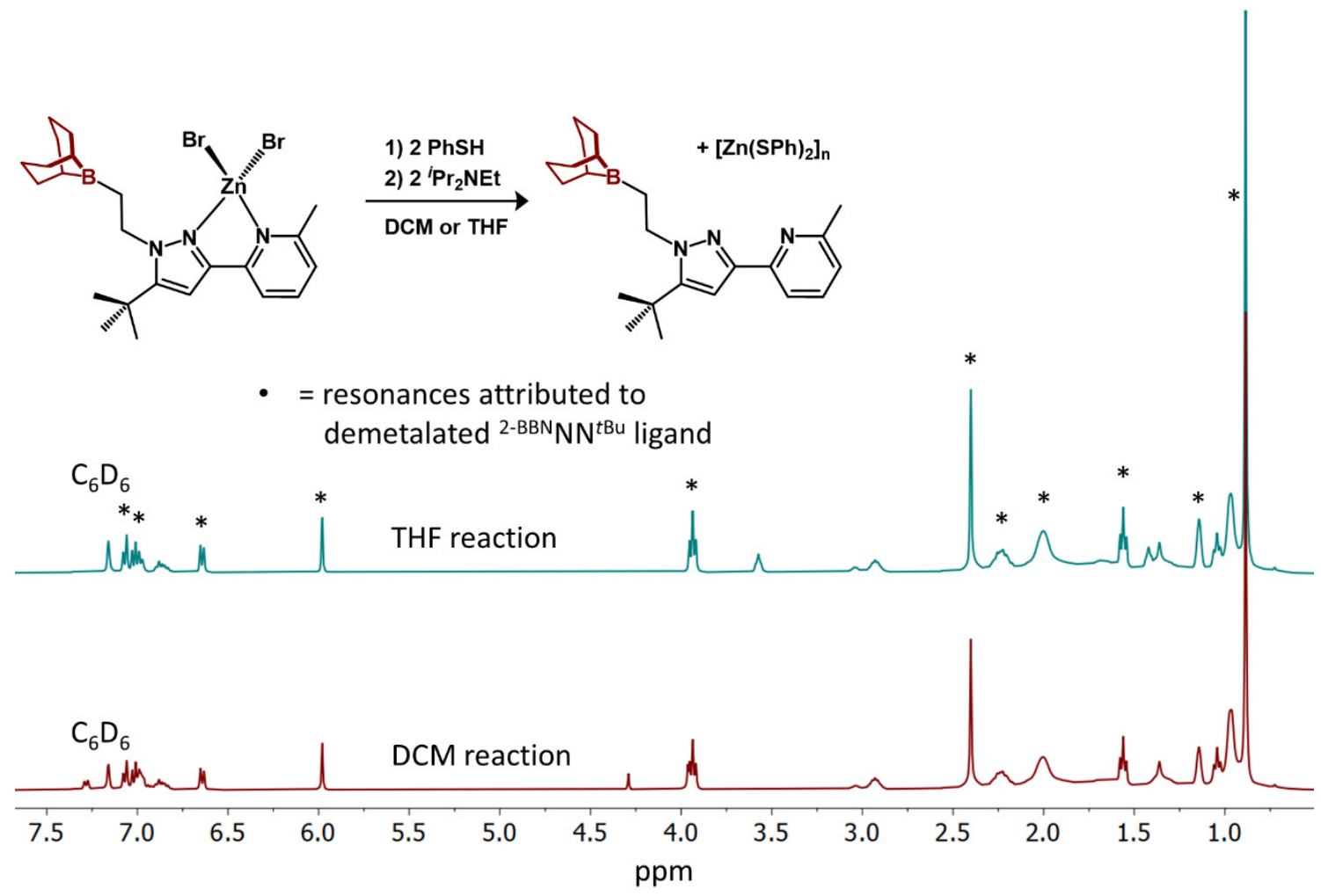

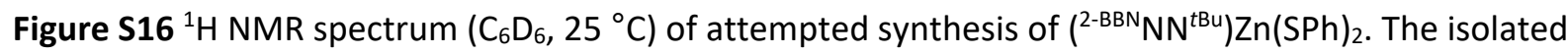
product is the demetalated ligand, ${ }^{2-B B N} \mathrm{NN}^{\mathrm{tBu}}$. Top: reaction in THF. Bottom: reaction in $\mathrm{CH}_{2} \mathrm{Cl}_{2}$. 

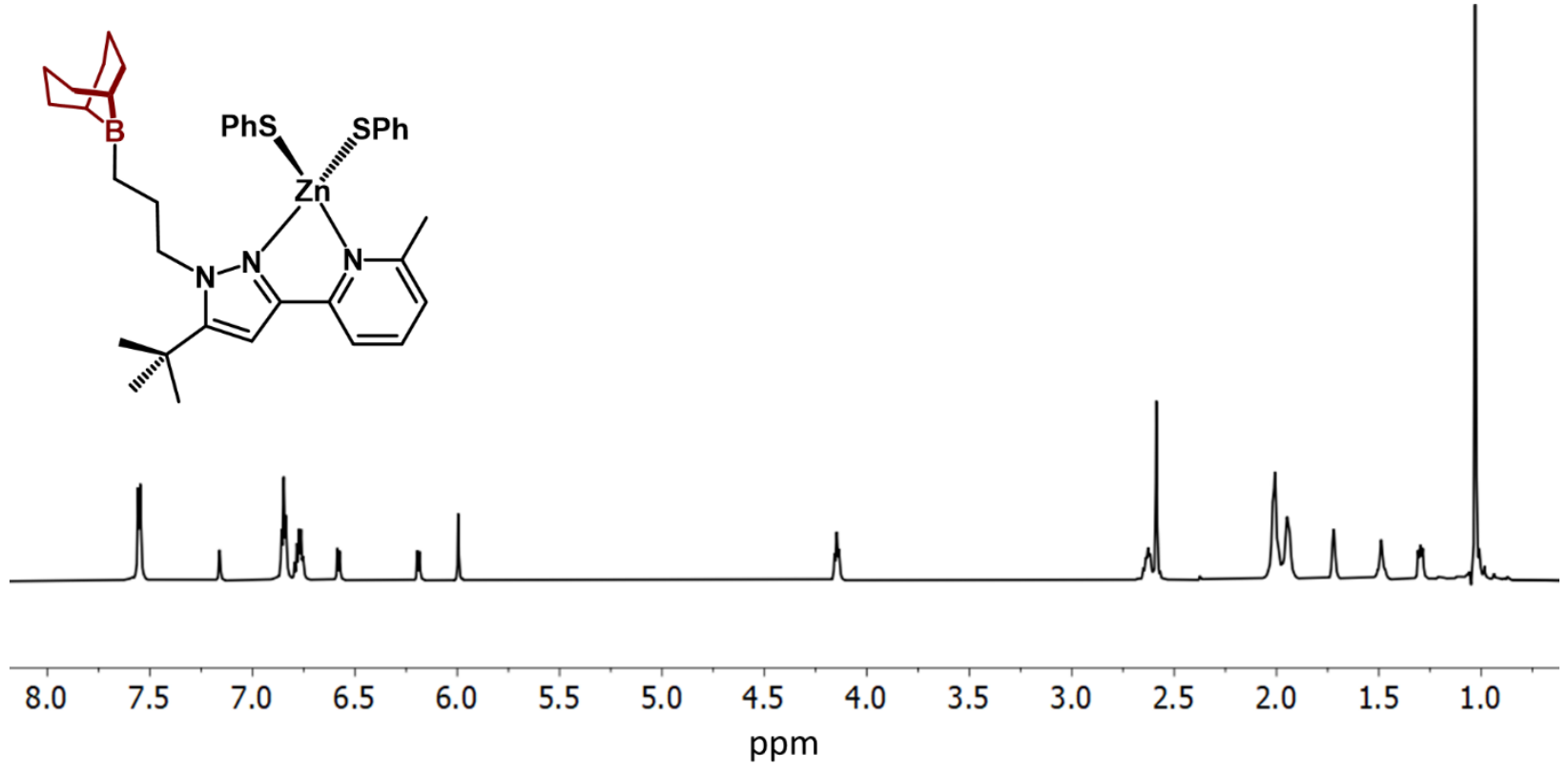

Figure $\mathrm{S} 17^{1} \mathrm{H}$ NMR spectrum $\left(\mathrm{C}_{6} \mathrm{D}_{6}, 25^{\circ} \mathrm{C}\right)$ of $\left({ }^{3-B B N} \mathrm{NN}^{\mathrm{tBu}}\right) \mathrm{Zn}(\mathrm{SPh})_{2}$.

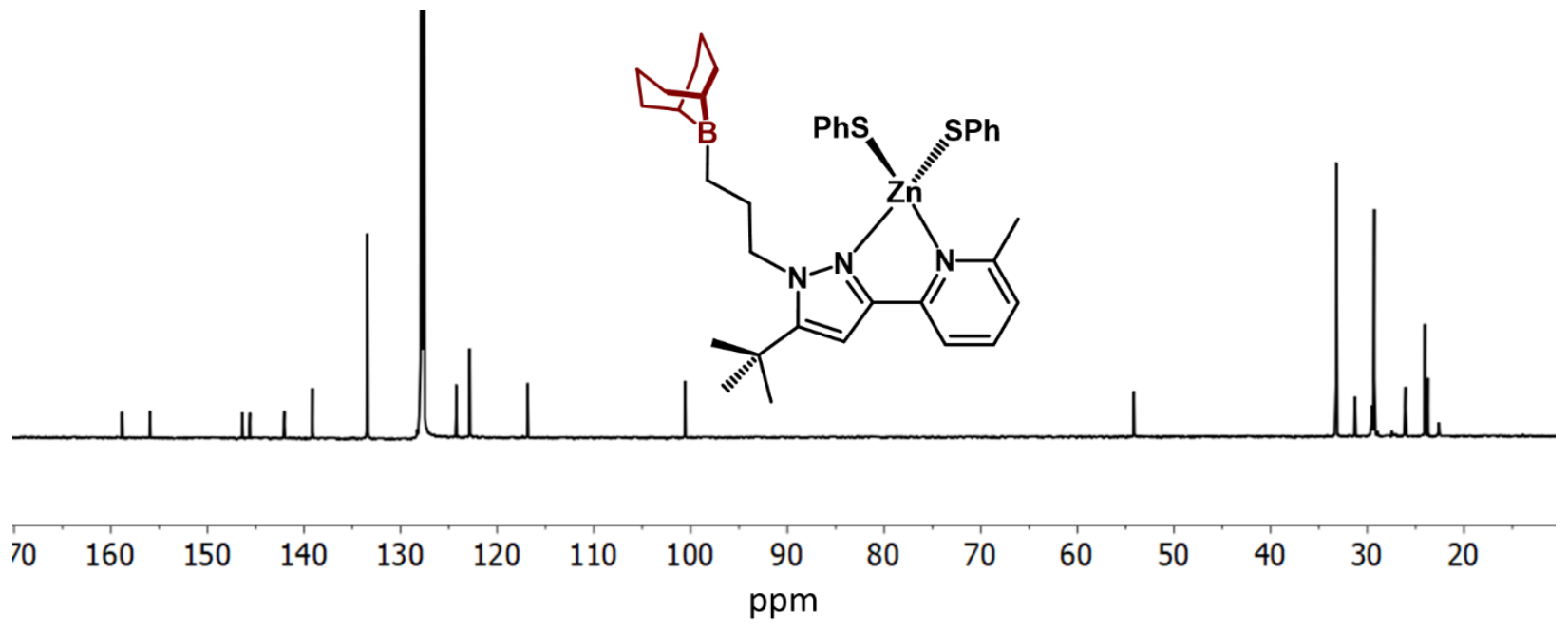

Figure $\mathrm{S} 18{ }^{13} \mathrm{C}$ NMR spectrum $\left(\mathrm{C}_{6} \mathrm{D}_{6}, 25^{\circ} \mathrm{C}\right)$ of $\left({ }^{3-B B N} \mathrm{NN}^{t \mathrm{Bu}}\right) \mathrm{Zn}(\mathrm{SPh})_{2}$. 


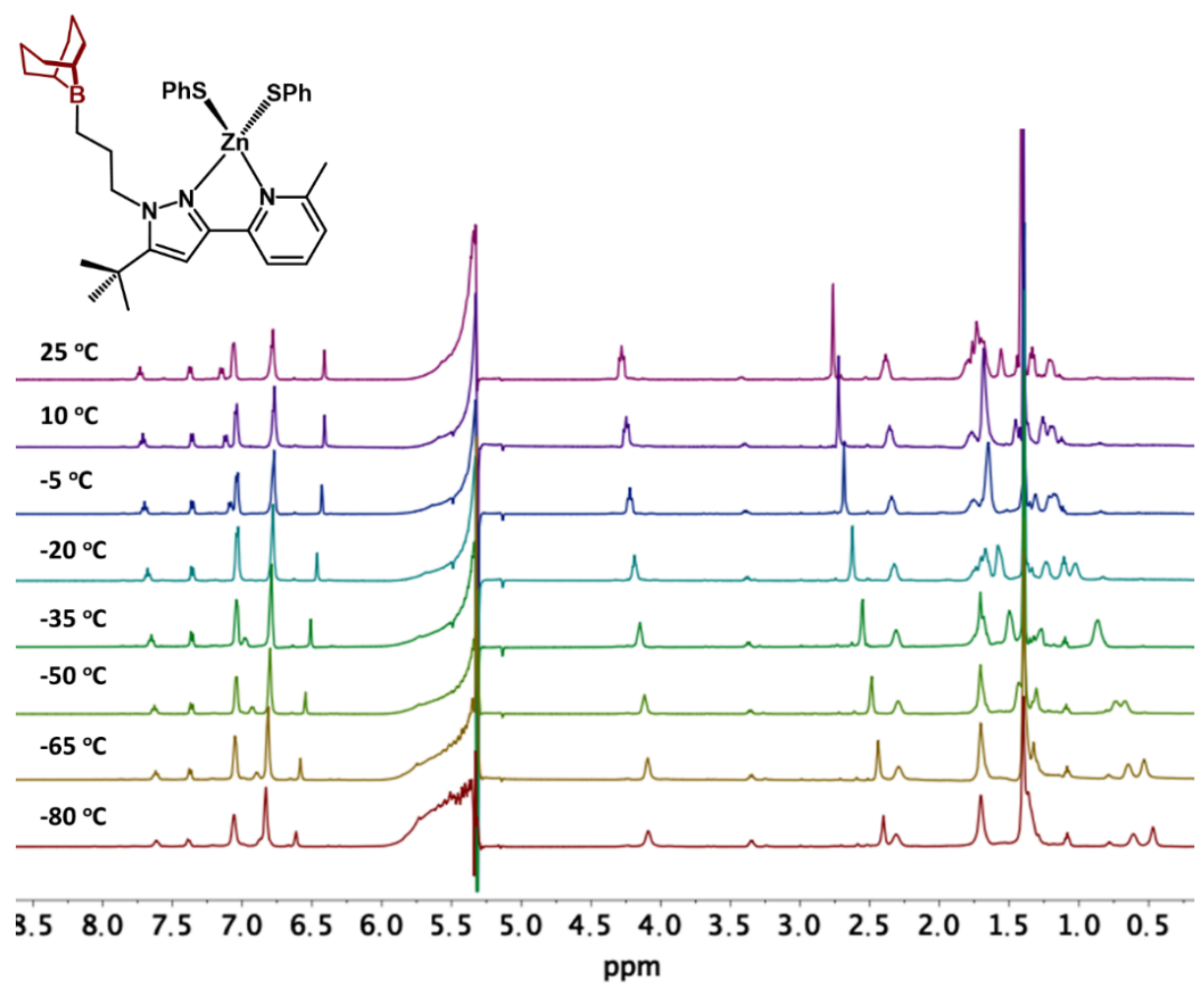

Figure S19 Variable temperature ${ }^{1} \mathrm{H}$ NMR spectra $\left(\mathrm{CH}_{2} \mathrm{Cl}_{2}\right)$ of $\left({ }^{3-B B N} \mathrm{NN}^{t B u}\right) \mathrm{Zn}(\mathrm{SPh})_{2}$.

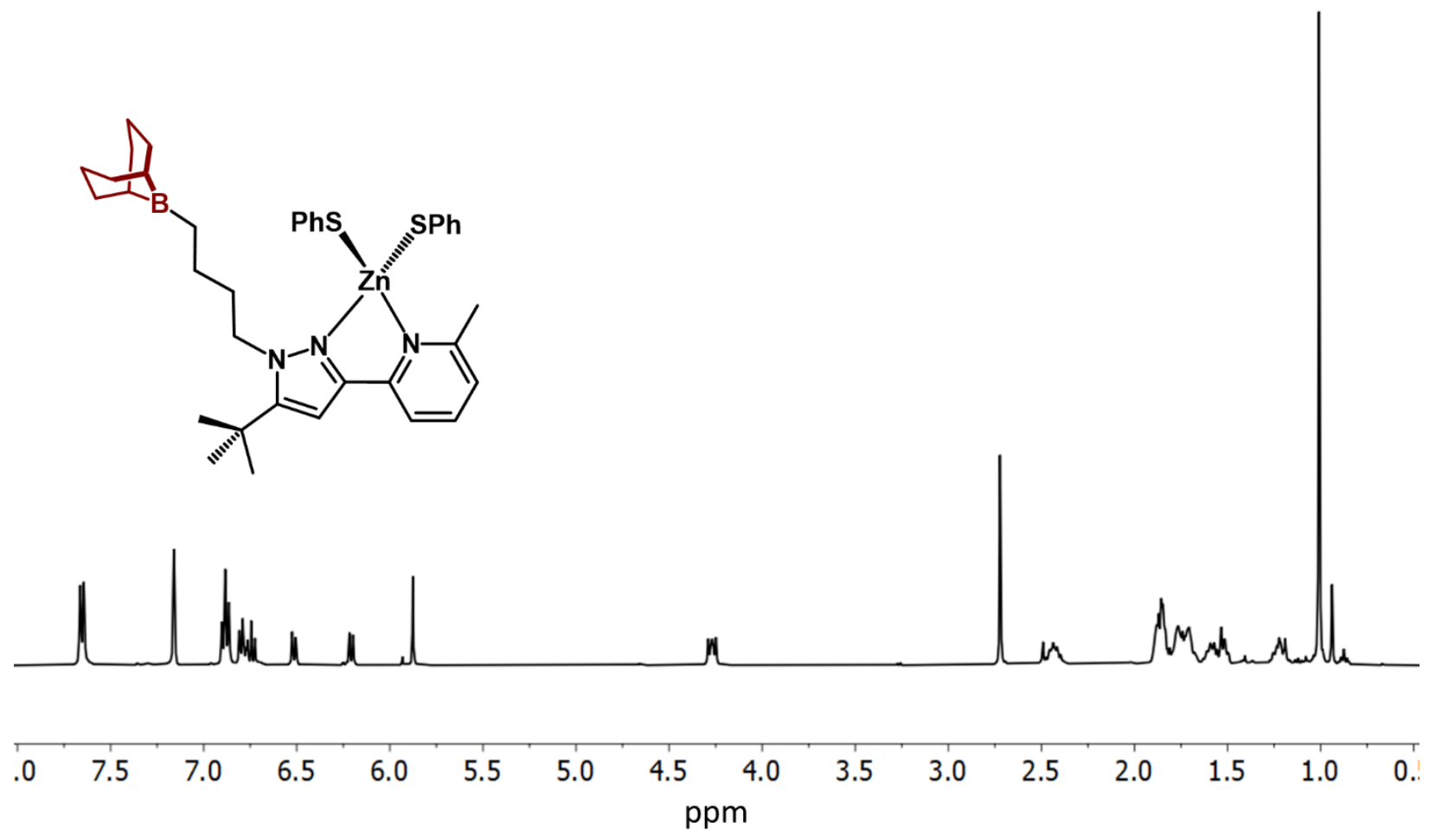

Figure $S 20{ }^{1} \mathrm{H}$ NMR spectrum $\left(\mathrm{C}_{6} \mathrm{D}_{6}, 25^{\circ} \mathrm{C}\right)$ of $\left({ }^{4-B B N} \mathrm{NN}^{\mathrm{tBu}}\right) \mathrm{Zn}(\mathrm{SPh})_{2}$. 

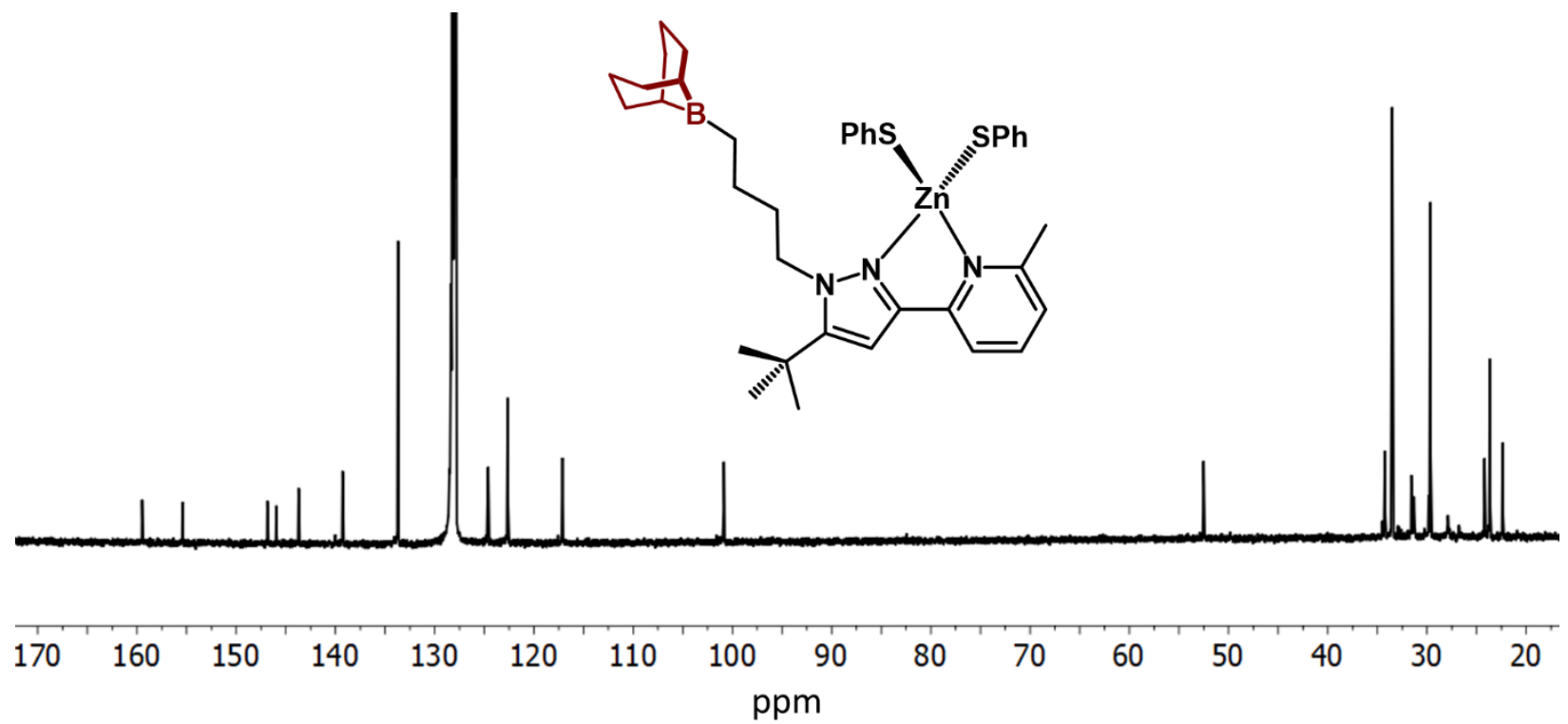

Figure $\mathrm{S} 21{ }^{13} \mathrm{C}$ NMR spectrum $\left(\mathrm{C}_{6} \mathrm{D}_{6}, 25^{\circ} \mathrm{C}\right)$ of $\left({ }^{4-B B N} \mathrm{NN}^{t \mathrm{Bu}}\right) \mathrm{Zn}(\mathrm{SPh})_{2}$.

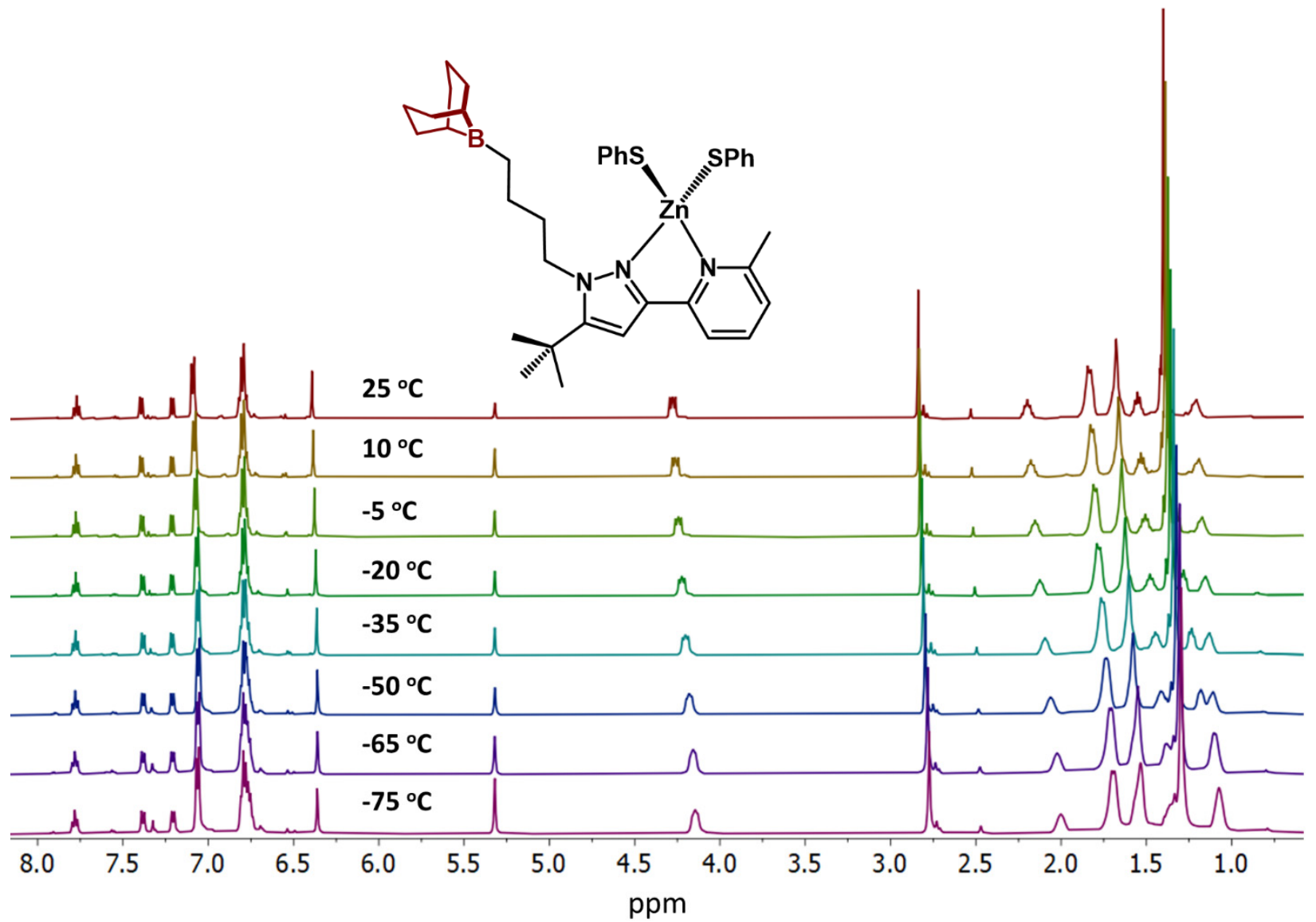

Figure S22 Variable temperature ${ }^{1} \mathrm{H}$ NMR spectra $\left(\mathrm{CD}_{2} \mathrm{Cl}_{2}\right)$ of $\left({ }^{4-B B N} \mathrm{NN}^{\mathrm{tBu}}\right) \mathrm{Zn}(\mathrm{SPh})_{2}$. 

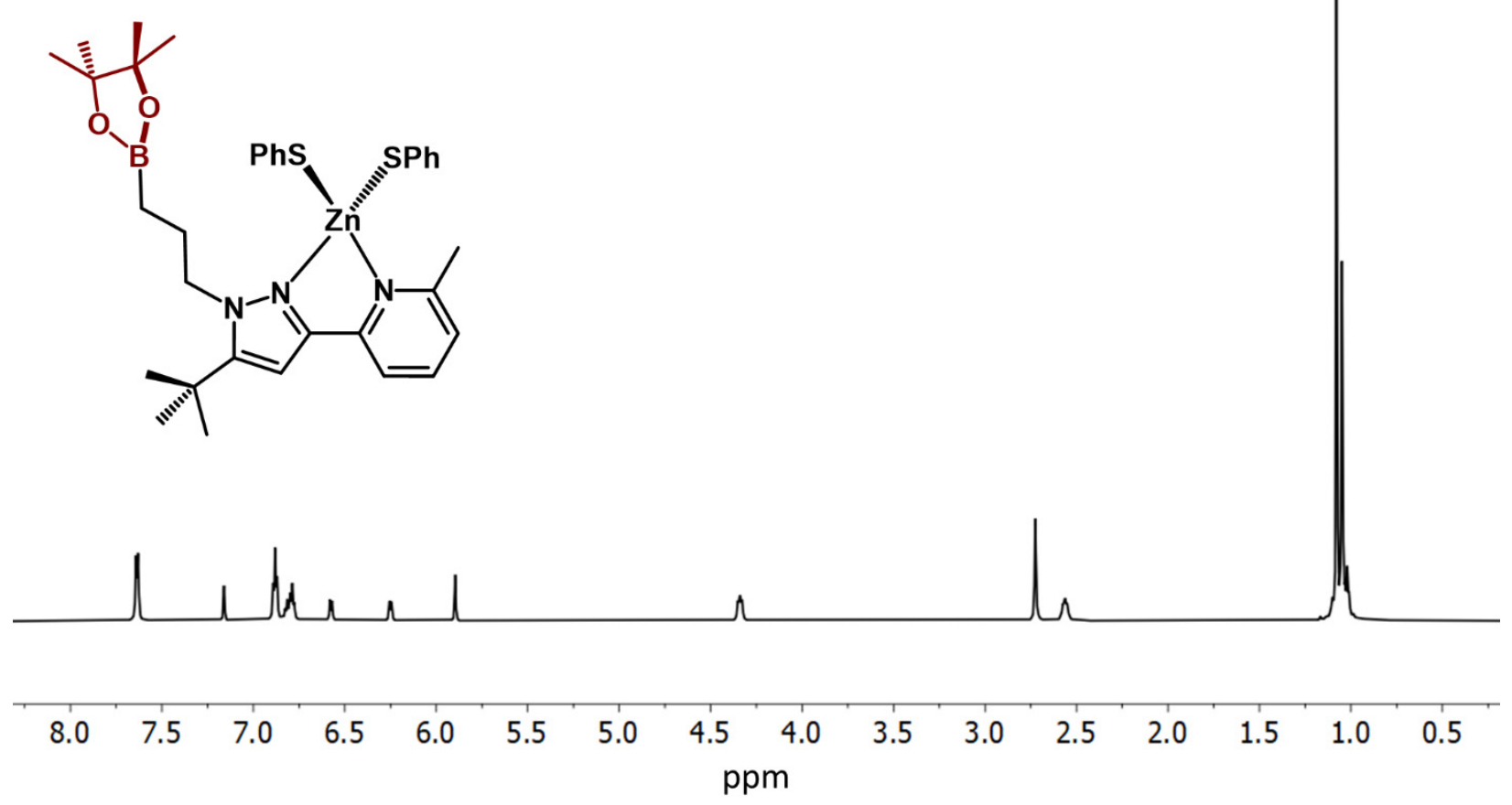

Figure $S 23{ }^{1} \mathrm{H}$ NMR spectrum $\left(\mathrm{C}_{6} \mathrm{D}_{6}, 25^{\circ} \mathrm{C}\right)$ of $\left({ }^{3-B P i n} \mathrm{NN}^{t \mathrm{Bu}}\right) \mathrm{Zn}(\mathrm{SPh})_{2}$.

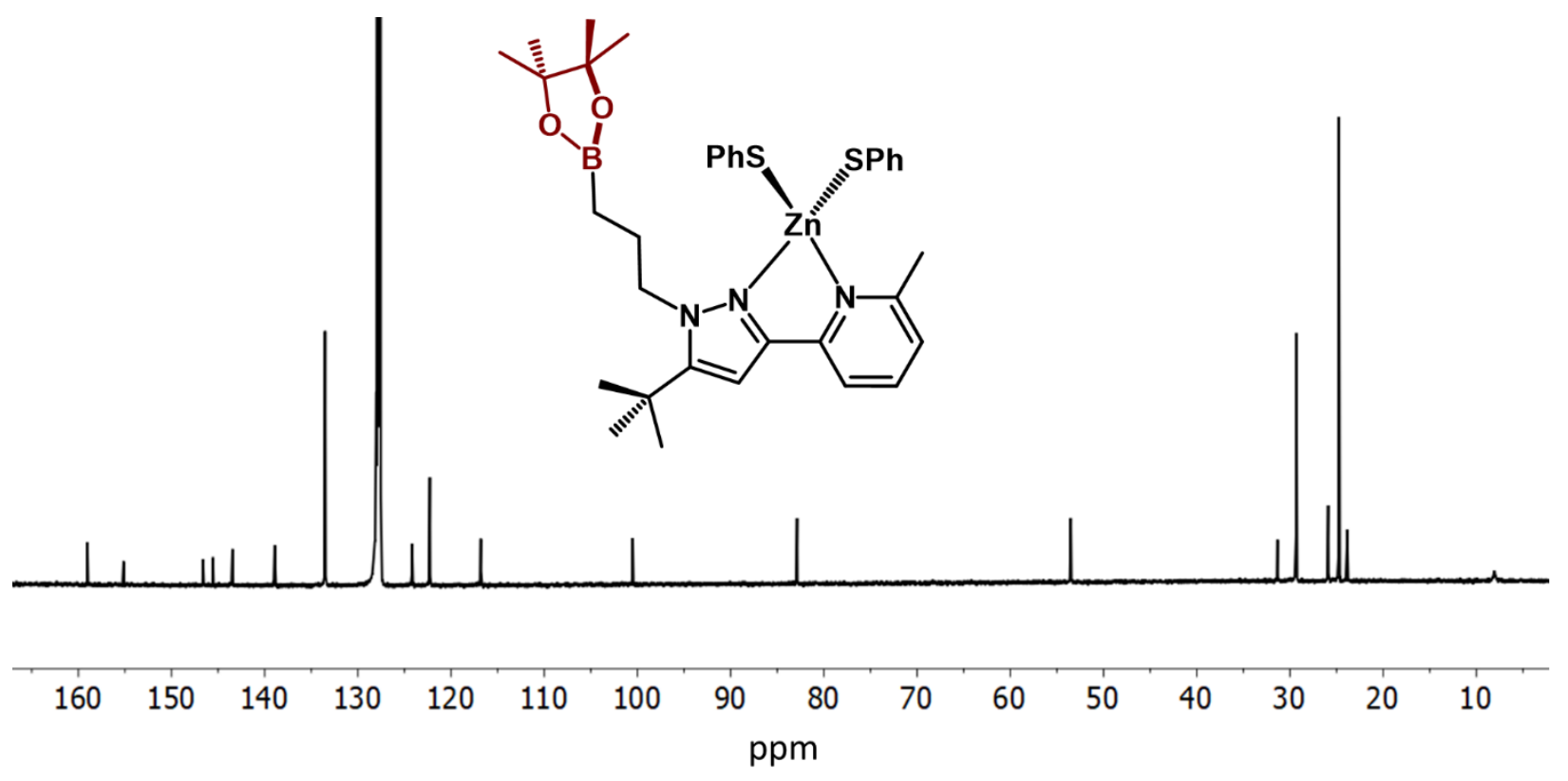

Figure S24 ${ }^{13} \mathrm{C}$ NMR spectrum $\left(\mathrm{C}_{6} \mathrm{D}_{6}, 25^{\circ} \mathrm{C}\right)$ of $\left(\left(^{3-B P i n} \mathrm{NN}^{\mathrm{tBu}}\right) \mathrm{Zn}(\mathrm{SPh})_{2}\right.$. 


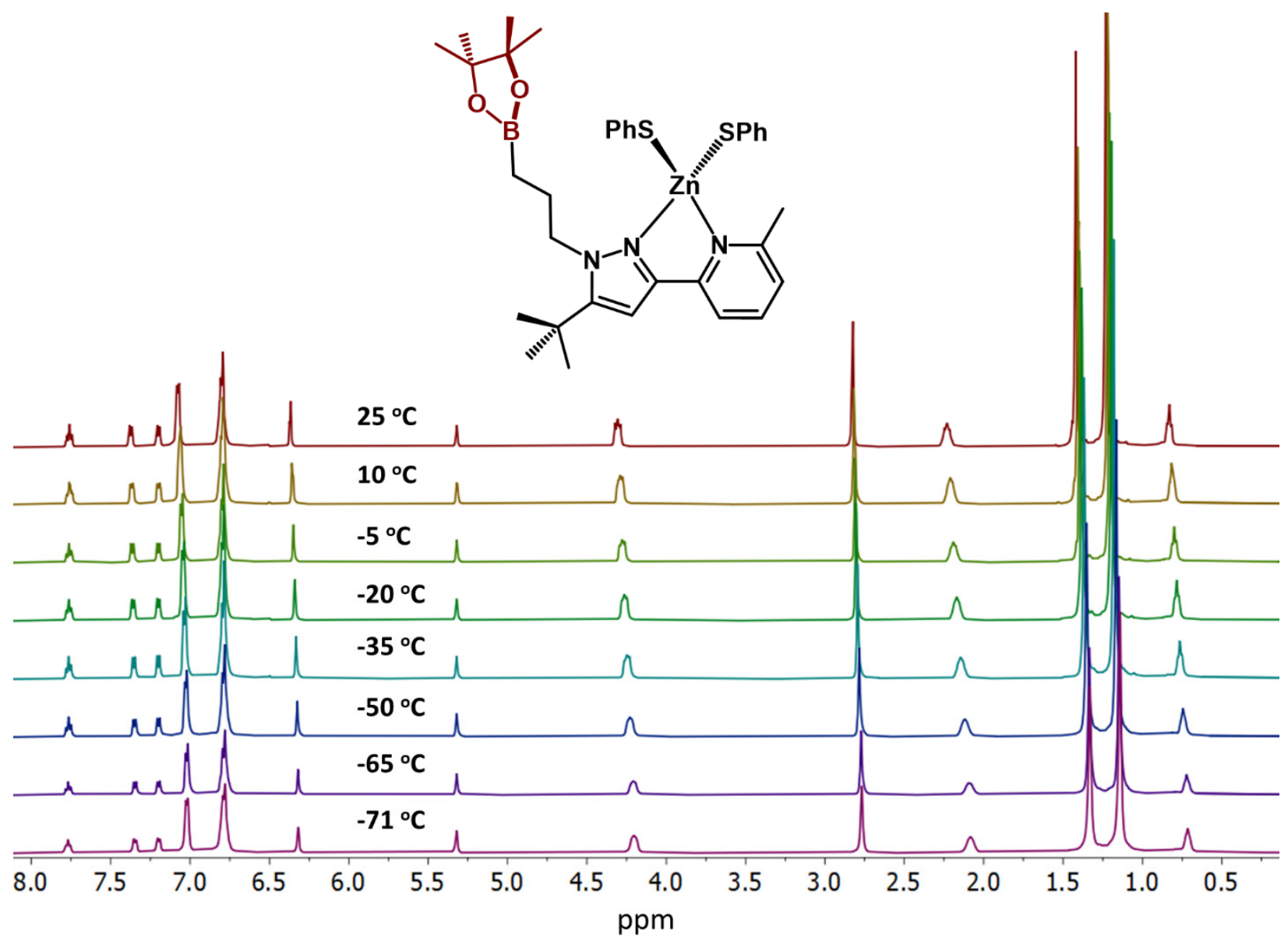

Figure S25 Variable temperature ${ }^{1} \mathrm{H}$ NMR spectra $\left(\mathrm{CD}_{2} \mathrm{Cl}_{2}\right)$ of $\left({ }^{3-B P i n} \mathrm{NN}^{t \mathrm{Bu}}\right) \mathrm{Zn}(\mathrm{SPh})_{2}$. 


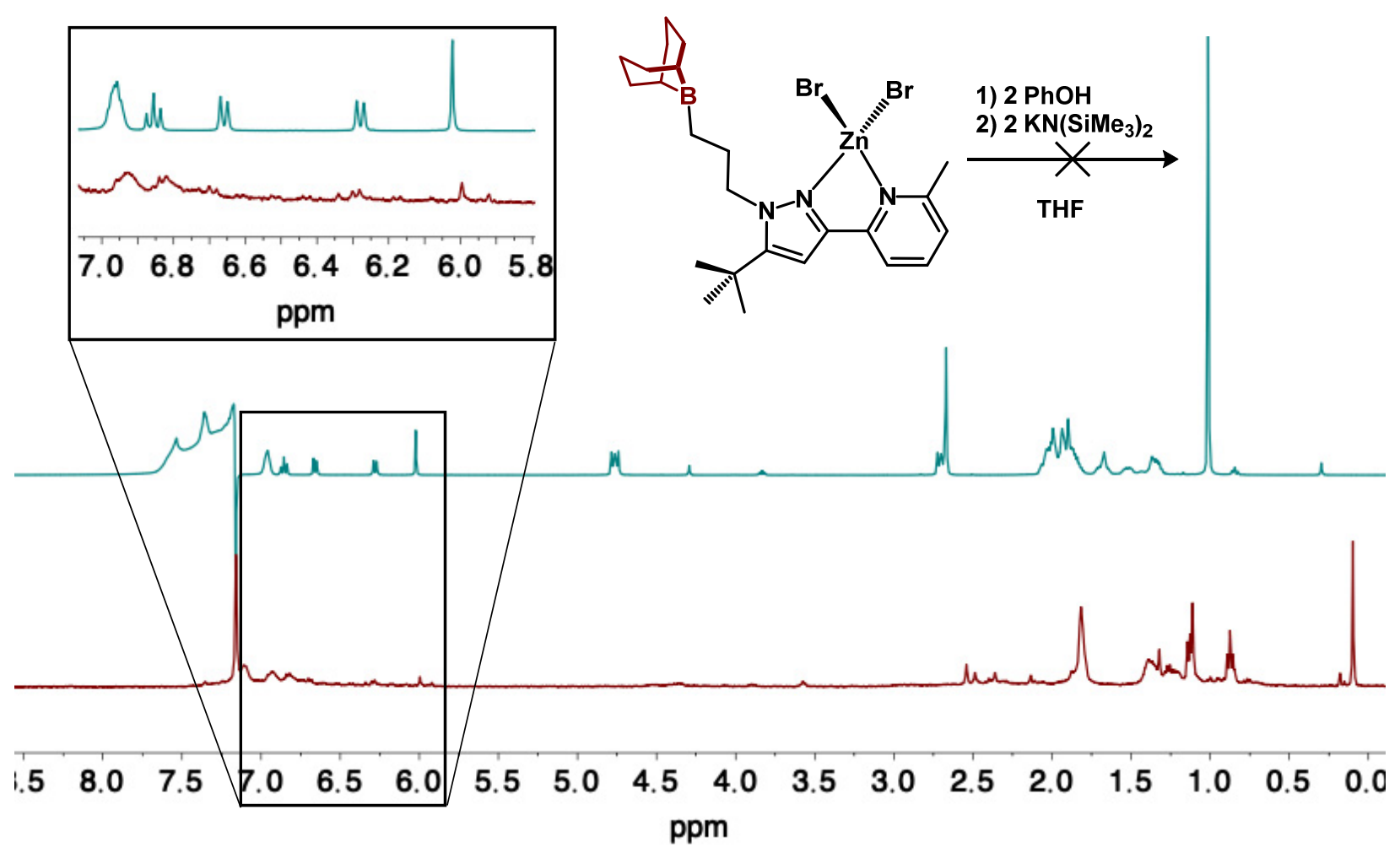

Figure S26 ${ }^{1} \mathrm{H}$ NMR spectrum of attempted synthesis of $\left({ }^{3-B B N} \mathrm{NN}^{t B u}\right) \mathrm{Zn}(\mathrm{OPh})_{2}$ resulting in an intractable

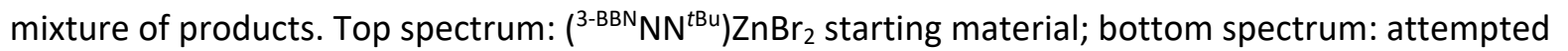
synthesis of $\left({ }^{3-B B N} N^{t B u}\right) Z n(O P h)_{2}$.

Tables S1 Shift $(\mathrm{Hz})$ of select resonances from VT ${ }^{1} \mathrm{H}$ NMR between the ranges of $+25^{\circ} \mathrm{C}$ to $-80{ }^{\circ} \mathrm{C}$.

\begin{tabular}{|c|c|c|c|c|c|c|c|}
\hline & $\begin{array}{c}p \text {-pyr-CH } \\
\Delta(\mathrm{Hz})\end{array}$ & $\begin{array}{l}\mathrm{pz}-\mathrm{CH} \\
\Delta(\mathrm{Hz})\end{array}$ & $\begin{array}{l}\mathrm{N}-\mathrm{CH}_{2} \\
\Delta(\mathrm{Hz})\end{array}$ & $\begin{array}{c}\text { pyr- } \mathrm{CH}_{3} \\
\Delta(\mathrm{Hz})\end{array}$ & $\begin{array}{l}\mathrm{C}\left(\mathrm{CH}_{3}\right)_{3} \\
\Delta(\mathrm{Hz})\end{array}$ & $\begin{array}{l}\text { PhS-H } \\
\Delta(H z)\end{array}$ & $\begin{array}{c}\text { THF } \\
\Delta(H z)\end{array}$ \\
\hline$\left({ }^{3-\mathrm{BPin}} \mathrm{NN}^{\mathrm{tBu}}\right) \mathrm{ZnBr}_{2}$ & 1.6 & 17.5 & 63.0 & 49.8 & 50.7 & -- & -- \\
\hline$\left({ }^{2-B B N} \mathrm{NN}^{\mathrm{tBu}}\right) \mathrm{ZnBr}_{2}$ & 5.7 & 18.9 & 75.3 & 55.9 & 50.9 & -- & 8.5 \\
\hline$\left({ }^{2-B B N} \mathrm{NN}^{\mathrm{tBu}}\right) \mathrm{ZnBr}_{2}+\mathrm{PhSH}$ & 7.9 & 18.5 & 67.2 & 51.2 & 49.0 & 45.3 & 21.3 \\
\hline$\left({ }^{3-B B N} \mathrm{NN}^{\mathrm{tBu}}\right) \mathrm{ZnBr}_{2}$ & 0.4 & 14.2 & 67.9 & 54.4 & 54.0 & -- & -- \\
\hline$\left({ }^{3-B B N} \mathrm{NN}^{\mathrm{tBu}}\right) \mathrm{ZnBr}_{2}+\mathrm{PhSH}$ & 3.0 & 13.6 & 61.0 & 50.2 & 50.3 & 45.5 & - \\
\hline$\left({ }^{4-B B N} \mathrm{NN}^{\mathrm{tBu}}\right) \mathrm{ZnBr}_{2}$ & 2.0 & 12.6 & 57.4 & 49.0 & 49.1 & -- & -- \\
\hline$\left({ }^{4-B B N} \mathrm{NN}^{\mathrm{tBu}}\right) \mathrm{ZnBr}_{2}+\mathrm{PhSH}$ & 2.2 & 12.1 & 57.0 & 48.1 & 47.9 & 45.4 & -- \\
\hline$\left({ }^{3-\mathrm{BPin}} \mathrm{NN}^{\mathrm{tBu}}\right) \mathrm{Zn}(\mathrm{SPh})_{2}^{\mathrm{b}}$ & 4.3 & 24.9 & 51.1 & 28.7 & 45.0 & -- & -- \\
\hline$\left({ }^{3-B B N} N^{t B u}\right) Z n(S P h)_{2}$ & 58.6 & 100.6 & 96.1 & 181.2 & 7.4 & -- & -- \\
\hline$\left({ }^{4-B B N} N^{t B u}\right) Z n(S P h)_{2}{ }^{a}$ & 5.2 & 15.5 & 66.7 & 32.2 & 53.2 & -- & -- \\
\hline
\end{tabular}

aLowest temperature recorded: $-75^{\circ} \mathrm{C}$. bowest temperature recorded: $-71^{\circ} \mathrm{C}$. 
Tables S2 Shift $(\mathrm{Hz})$ of select resonances from VT ${ }^{1} \mathrm{H}$ NMR studies between the ranges of $+25^{\circ} \mathrm{C}$ to -65 ${ }^{\circ} \mathrm{C}$.

\begin{tabular}{|c|c|c|c|c|c|}
\hline & $\begin{array}{c}p \text {-pyr-CH } \\
\Delta(\mathrm{Hz})\end{array}$ & $\begin{array}{l}\mathrm{pz}-\mathrm{CH} \\
\Delta(\mathrm{Hz})\end{array}$ & $\begin{array}{l}\mathrm{N}-\mathrm{CH}_{2} \\
\Delta(\mathrm{Hz})\end{array}$ & $\begin{array}{c}\text { pyr- } \mathrm{CH}_{3} \\
\Delta(\mathrm{Hz})\end{array}$ & $\begin{array}{l}\mathrm{C}\left(\mathrm{CH}_{3}\right)_{3} \\
\Delta(\mathrm{Hz})\end{array}$ \\
\hline$\left(\left(^{3-B P i n} N^{t B u}\right) Z n(S P h)_{2}\right.$ & 4.1 & 24.8 & 47.8 & 25.5 & 42.1 \\
\hline$\left({ }^{3-B B N} N^{t B u}\right) Z n(S P h)_{2}$ & 56.3 & 84.8 & 93.3 & 162.1 & 9.2 \\
\hline$\left({ }^{4-B B N} N^{t B u}\right) Z n(S P h)_{2}$ & 4.9 & 16.2 & 60.6 & 27.8 & 48.4 \\
\hline
\end{tabular}

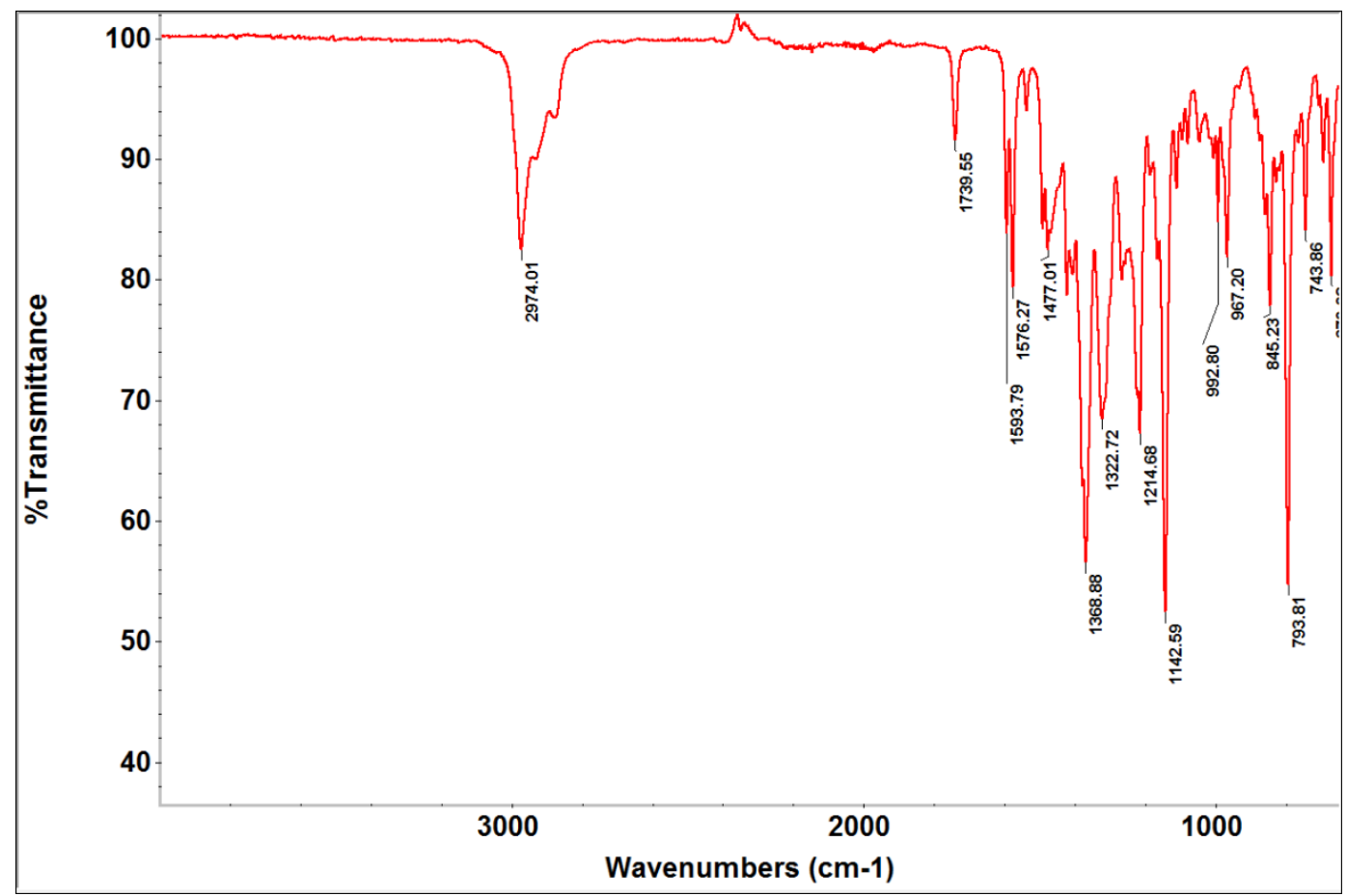

Figure S27 Infrared spectrum (ATR, neat) of 2-(5-(tert-butyl)-1-(3-(4,4,5,5-tetramethyl-1,3,2dioxaborolan-2-yl)propyl)-1H-pyrazol-3-yl)-6-methylpyridine $\left({ }^{3-B P i n} N^{t B u}\right)$. 


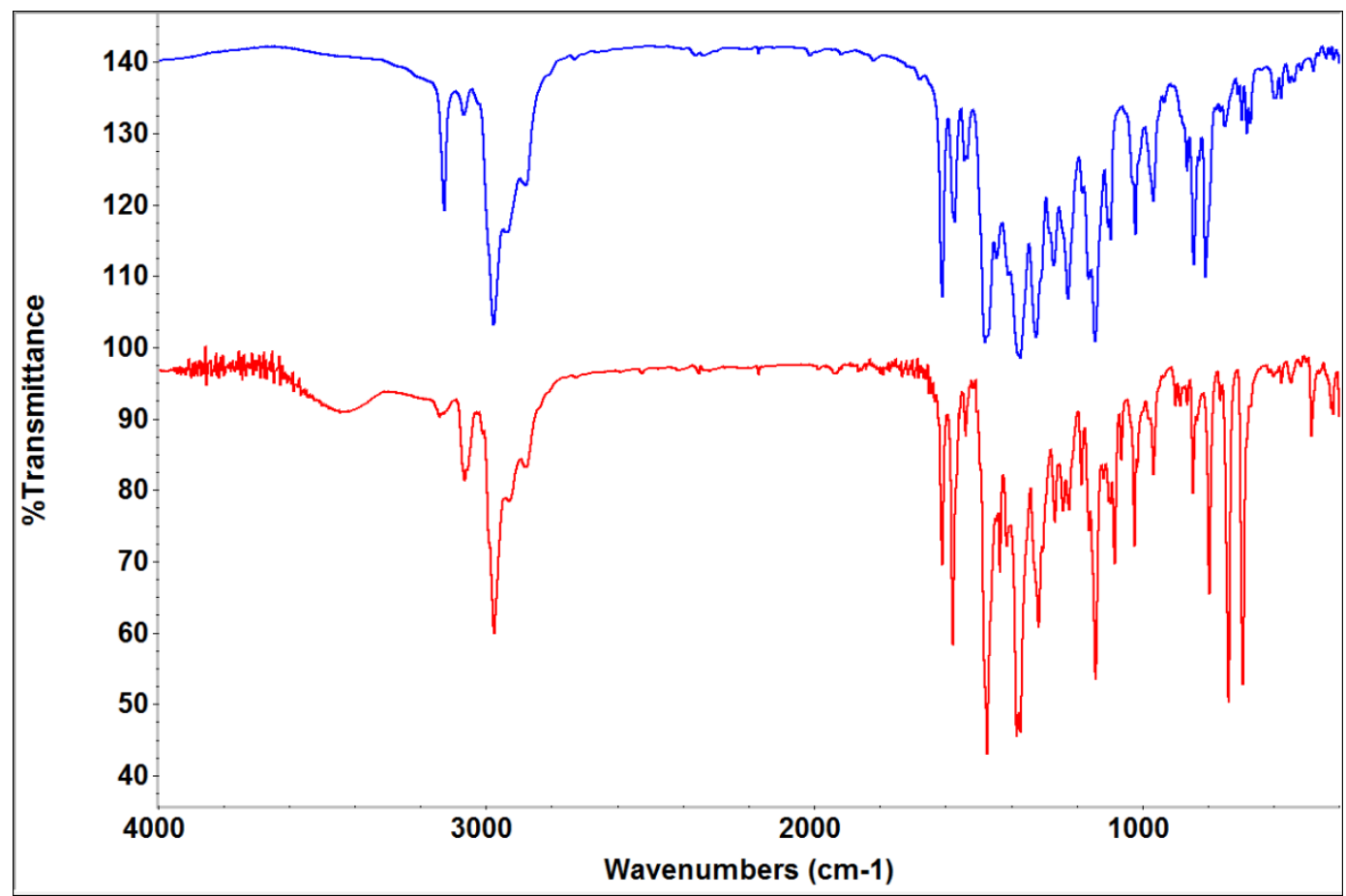

Figure S28 Infrared spectra $(\mathrm{KBr})$ of $\left({ }^{3-B P i n} \mathrm{NN}^{\mathrm{tBu}}\right) \mathrm{ZnBr}_{2}$ (top, blue) and $\left({ }^{3-B P i n} \mathrm{NN}^{\mathrm{tBu}}\right) \mathrm{Zn}(\mathrm{SPh})_{2}$ (bottom, red).

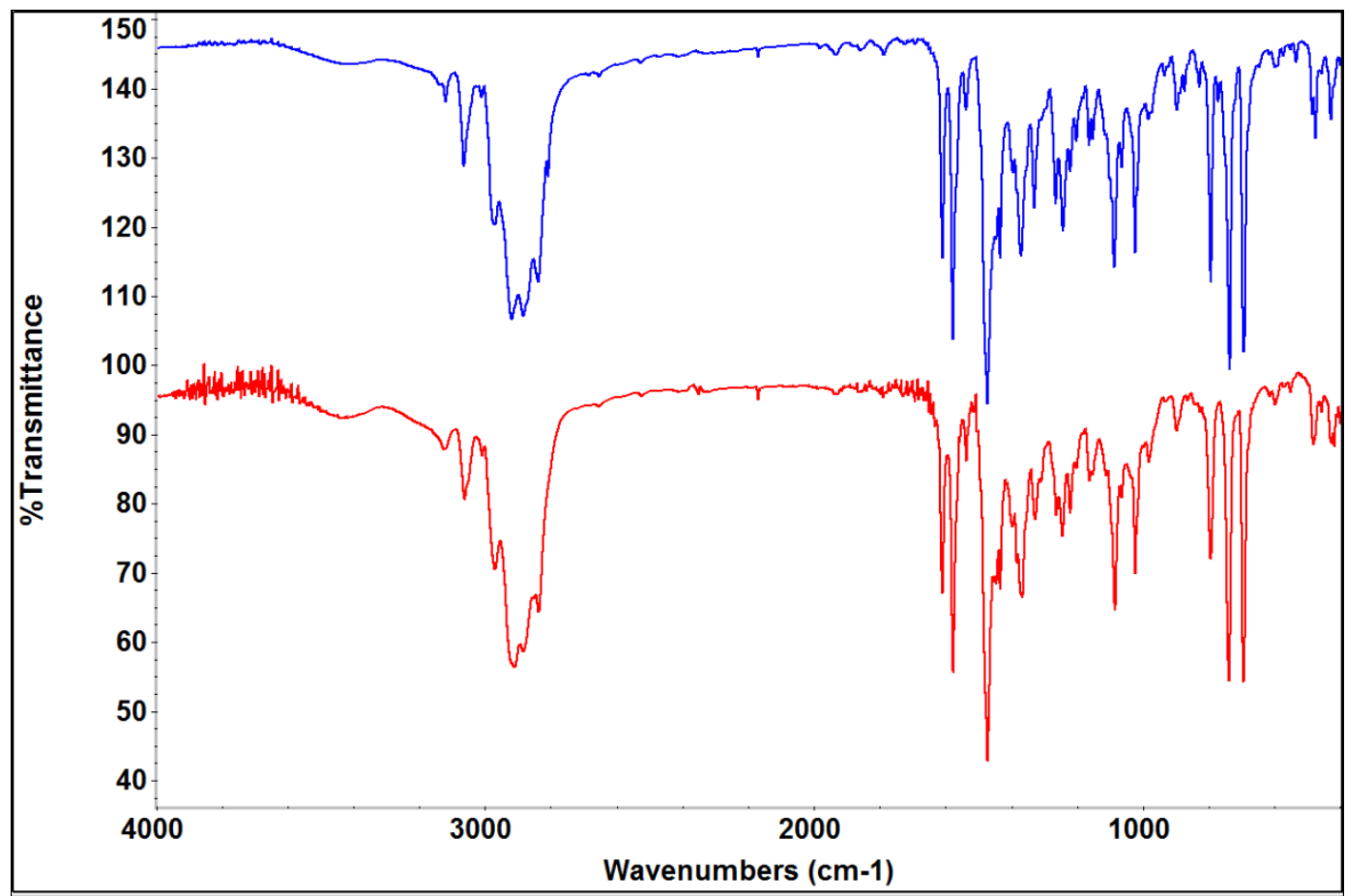

Figure S29 Infrared spectra $(\mathrm{KBr})$ of $\left({ }^{3-B B N} \mathrm{NN}^{\mathrm{tBu}}\right) \mathrm{Zn}(\mathrm{SPh})_{2}$ (top, blue) and $\left({ }^{4-B B N} \mathrm{NN}^{\mathrm{tBu}}\right) \mathrm{Zn}(\mathrm{SPh})_{2}$ (bottom, red). 
Local name: JK5230

CCDC: 2073452

Table S3 Experimental parameters for $\left({ }^{3-B P i n} \mathrm{NN}^{t B u}\right) \mathrm{ZnBr}_{2}$.

\begin{tabular}{|c|c|}
\hline \multicolumn{2}{|l|}{ Crystal data } \\
\hline Chemical formula & $\mathrm{C}_{22} \mathrm{H}_{34} \mathrm{BBr}_{2} \mathrm{~N}_{3} \mathrm{O}_{2} \mathrm{Zn}$ \\
\hline$M_{\mathrm{r}}$ & 608.52 \\
\hline Crystal system, space group & Monoclinic, $P 2_{1} / n$ \\
\hline Temperature (K) & 150 \\
\hline$a, b, c(\AA)$ & $12.8763(7), 15.5516(10), 13.3477(8)$ \\
\hline$\beta\left(^{\circ}\right)$ & $104.089(2)$ \\
\hline$V\left(\AA^{3}\right)$ & 2592.4 (3) \\
\hline$z$ & 4 \\
\hline Radiation type & Mo $K \alpha$ \\
\hline$\mu\left(\mathrm{mm}^{-1}\right)$ & 4.05 \\
\hline Crystal size $(\mathrm{mm})$ & $0.48 \times 0.21 \times 0.14$ \\
\hline \multicolumn{2}{|l|}{ Data collection } \\
\hline Diffractometer & Bruker AXS D8 Quest Photon 100 CMOS diffractometer \\
\hline Absorption correction & $\begin{array}{l}\text { Multi-scan SADABS 2016/2: Krause, L., Herbst-Irmer, R., } \\
\text { Sheldrick G.M. \& Stalke D., J. Appl. Cryst. } 48 \text { (2015) 3-10 }\end{array}$ \\
\hline$T_{\min }, T_{\max }$ & $0.387,0.747$ \\
\hline $\begin{array}{l}\text { No. of measured, independent and } \\
\text { observed }[I>2 \mathrm{~s}(I)] \text { reflections }\end{array}$ & $50445,9932,6790$ \\
\hline$R_{\text {int }}$ & 0.052 \\
\hline$(\sin \theta / \lambda)_{\max }\left(\AA^{-1}\right)$ & 0.771 \\
\hline \multicolumn{2}{|l|}{ Refinement } \\
\hline$R\left[F^{2}>2 \sigma\left(F^{2}\right)\right], w R\left(F^{2}\right), S$ & $0.038,0.095,1.02$ \\
\hline No. of reflections & 9932 \\
\hline No. of parameters & 288 \\
\hline $\mathrm{H}$-atom treatment & $\mathrm{H}$-atom parameters constrained \\
\hline$\Delta \rho_{\max }, \Delta \rho_{\min }\left(\mathrm{e} \AA^{-3}\right)$ & $0.70,-0.85$ \\
\hline
\end{tabular}

Computer programs: Apex3 v2018.7-2 (Bruker, 2018), SAINT V8.38A (Bruker, 2018), SHELXS97

(Sheldrick, 2008), SHELXL2018/3 (Sheldrick, 2015, 2018), SHELXLE Rev1030 (Hübschle et al., 2011). 


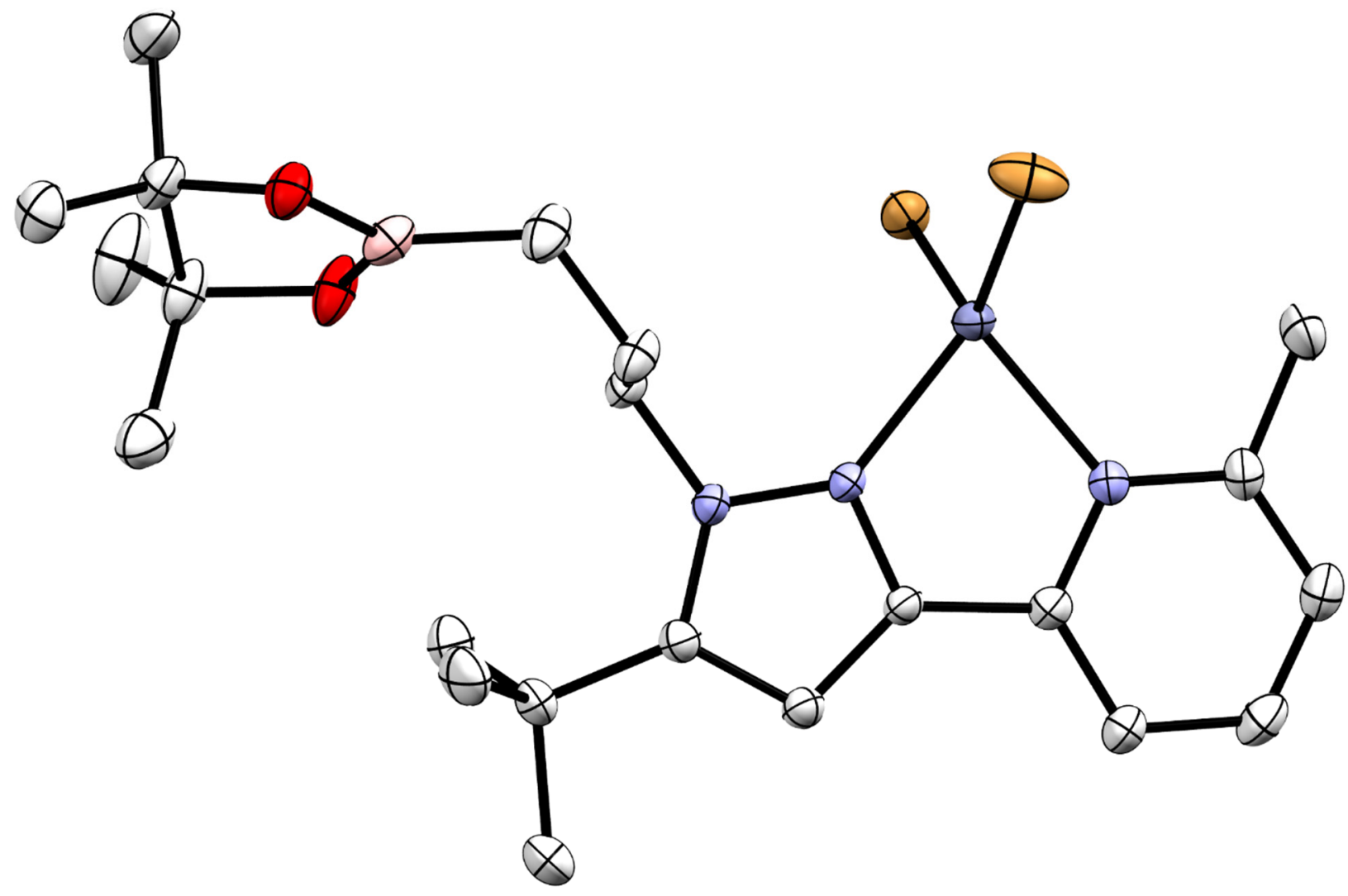

Figure S30 Molecular structure of $\left({ }^{3-B P i n} \mathrm{NN}^{\mathrm{tBu}}\right) \mathrm{ZnBr}_{2}$ displayed with $50 \%$ probability ellipsoids. Hydrogen atoms are omitted for clarity. 
Complex: $\left({ }^{3-B B N} N^{t B u}\right) Z n(S P h)_{2}$

Local name: EN2215

CCDC: 2073450

Table S4 Experimental parameters for $\left({ }^{3-B B N} \mathrm{NN}^{t B u}\right) \mathrm{Zn}(\mathrm{SPh})_{2}$.

\begin{tabular}{|c|c|}
\hline \multicolumn{2}{|l|}{ Crystal data } \\
\hline Chemical formula & $\mathrm{C}_{36} \mathrm{H}_{46} \mathrm{BN}_{3} \mathrm{~S}_{2} \mathrm{Zn}$ \\
\hline$M_{\mathrm{r}}$ & 661.06 \\
\hline Crystal system, space group & Monoclinic, $P 2_{1} / n$ \\
\hline Temperature (K) & 150 \\
\hline$a, b, c(\AA)$ & 14.8525 (8), $11.4911(6), 19.7559$ (9) \\
\hline$\beta\left(^{\circ}\right)$ & $97.420(2)$ \\
\hline$V\left(\AA^{3}\right)$ & $3343.5(3)$ \\
\hline$Z$ & 4 \\
\hline Radiation type & Mo $K \alpha$ \\
\hline$\mu\left(\mathrm{mm}^{-1}\right)$ & 0.89 \\
\hline Crystal size $(\mathrm{mm})$ & $0.36 \times 0.32 \times 0.25$ \\
\hline \multicolumn{2}{|l|}{ Data collection } \\
\hline Diffractometer & Bruker AXS D8 Quest Photon 100 CMOS diffractometer \\
\hline Absorption correction & $\begin{array}{l}\text { Multi-scan SADABS 2016/2: Krause, L., Herbst-Irmer, R., Sheldrick } \\
\text { G.M. \& Stalke D., J. Appl. Cryst. } 48 \text { (2015) 3-10 }\end{array}$ \\
\hline$T_{\min }, T_{\max }$ & $0.687,0.747$ \\
\hline $\begin{array}{l}\text { No. of measured, independent and } \\
\text { observed }[I>2 s(I)] \text { reflections }\end{array}$ & $55642,12483,8381$ \\
\hline$R_{\text {int }}$ & 0.052 \\
\hline$(\sin \theta / \lambda)_{\max }\left(\AA^{-1}\right)$ & 0.770 \\
\hline \multicolumn{2}{|l|}{ Refinement } \\
\hline$R\left[F^{2}>2 \sigma\left(F^{2}\right)\right], w R\left(F^{2}\right), S$ & $0.041,0.099,1.01$ \\
\hline No. of reflections & 12483 \\
\hline No. of parameters & 392 \\
\hline $\mathrm{H}$-atom treatment & $\mathrm{H}$-atom parameters constrained \\
\hline$\Delta \rho_{\max }, \Delta \rho_{\min }\left(\mathrm{e} \AA^{-3}\right)$ & $0.59,-0.51$ \\
\hline
\end{tabular}

Computer programs: Apex3 v2018.7-2 (Bruker, 2018), SAINT V8.38A (Bruker, 2018), SHELXS97

(Sheldrick, 2008), SHELXL2018/3 (Sheldrick, 2015, 2018), SHELXLE Rev1030 (Hübschle et al., 2011). 


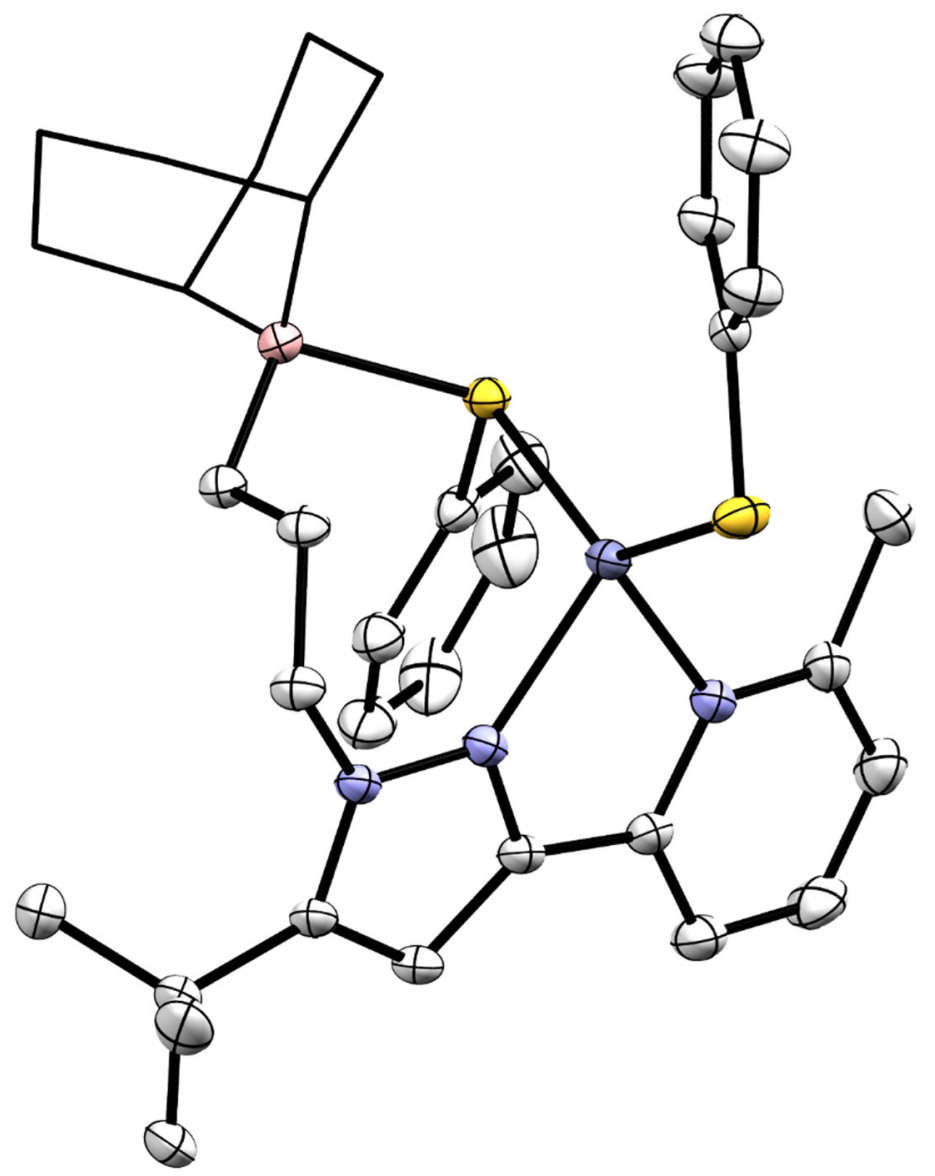

Figure S31 Molecular structure of $\left({ }^{3-B B N} \mathrm{NN}^{t B u}\right) \mathrm{Zn}(\mathrm{SPh})_{2}$ displayed with $50 \%$ probability ellipsoids.

Hydrogen atoms are omitted for clarity. The 9-BBN substituent is displayed in wireframe for improved clarity. 
Complex: $\left({ }^{4-B B N} N^{t B u}\right) Z n(S P h)_{2}$

Local name: JK5276

CCDC: 2073453

Table S5 Experimental parameters for $\left({ }^{4-B B N} \mathrm{NN}^{t B u}\right) \mathrm{Zn}(\mathrm{SPh})_{2}$.

\begin{tabular}{|c|c|}
\hline \multicolumn{2}{|l|}{ Crystal data } \\
\hline Chemical formula & $\mathrm{C}_{37} \mathrm{H}_{48} \mathrm{BN}_{3} \mathrm{~S}_{2} \mathrm{Zn}$ \\
\hline$M_{\mathrm{r}}$ & 675.08 \\
\hline Crystal system, space group & Monoclinic, $P 2_{1} / c$ \\
\hline Temperature $(\mathrm{K})$ & 150 \\
\hline$a, b, c(\AA)$ & 19.3308 (7), $10.8659(3), 17.5242$ (6) \\
\hline$\beta\left(^{\circ}\right)$ & 104.7875 (13) \\
\hline$V\left(\AA^{3}\right)$ & $3559.0(2)$ \\
\hline$Z$ & 4 \\
\hline Radiation type & Mo $K \alpha$ \\
\hline$\mu\left(\mathrm{mm}^{-1}\right)$ & 0.84 \\
\hline Crystal size $(\mathrm{mm})$ & $0.41 \times 0.36 \times 0.31$ \\
\hline \multicolumn{2}{|l|}{ Data collection } \\
\hline Diffractometer & $\begin{array}{l}\text { Bruker AXS D8 Quest diffractometer with Photonll charge- } \\
\text { integrating pixel array detector (CPAD) }\end{array}$ \\
\hline Absorption correction & $\begin{array}{l}\text { Multi-scan SADABS 2016/2: Krause, L., Herbst-Irmer, R., Sheldrick } \\
\text { G.M. \& Stalke D., J. Appl. Cryst. } 48 \text { (2015) 3-10 }\end{array}$ \\
\hline$T_{\min }, T_{\max }$ & $0.688,0.747$ \\
\hline $\begin{array}{l}\text { No. of measured, independent and } \\
\text { observed }[I>2 s(I)] \text { reflections }\end{array}$ & $62035,12986,9462$ \\
\hline$R_{\text {int }}$ & 0.043 \\
\hline$(\sin \theta / \lambda)_{\max }\left(\AA^{-1}\right)$ & 0.770 \\
\hline \multicolumn{2}{|l|}{ Refinement } \\
\hline$R\left[F^{2}>2 \sigma\left(F^{2}\right)\right], w R\left(F^{2}\right), S$ & $0.040,0.108,1.02$ \\
\hline No. of reflections & 12986 \\
\hline No. of parameters & 401 \\
\hline $\mathrm{H}$-atom treatment & $\mathrm{H}$-atom parameters constrained \\
\hline$\Delta \rho_{\max }, \Delta \rho_{\min }\left(\mathrm{e} \AA^{-3}\right)$ & $0.89,-0.49$ \\
\hline
\end{tabular}

Computer programs: Apex3 v2018.7-2 (Bruker, 2018), SAINT V8.38A (Bruker, 2018), SHELXS97

(Sheldrick, 2008), SHELXL2018/3 (Sheldrick, 2015, 2018), SHELXLE Rev1030 (Hübschle et al., 2011). 


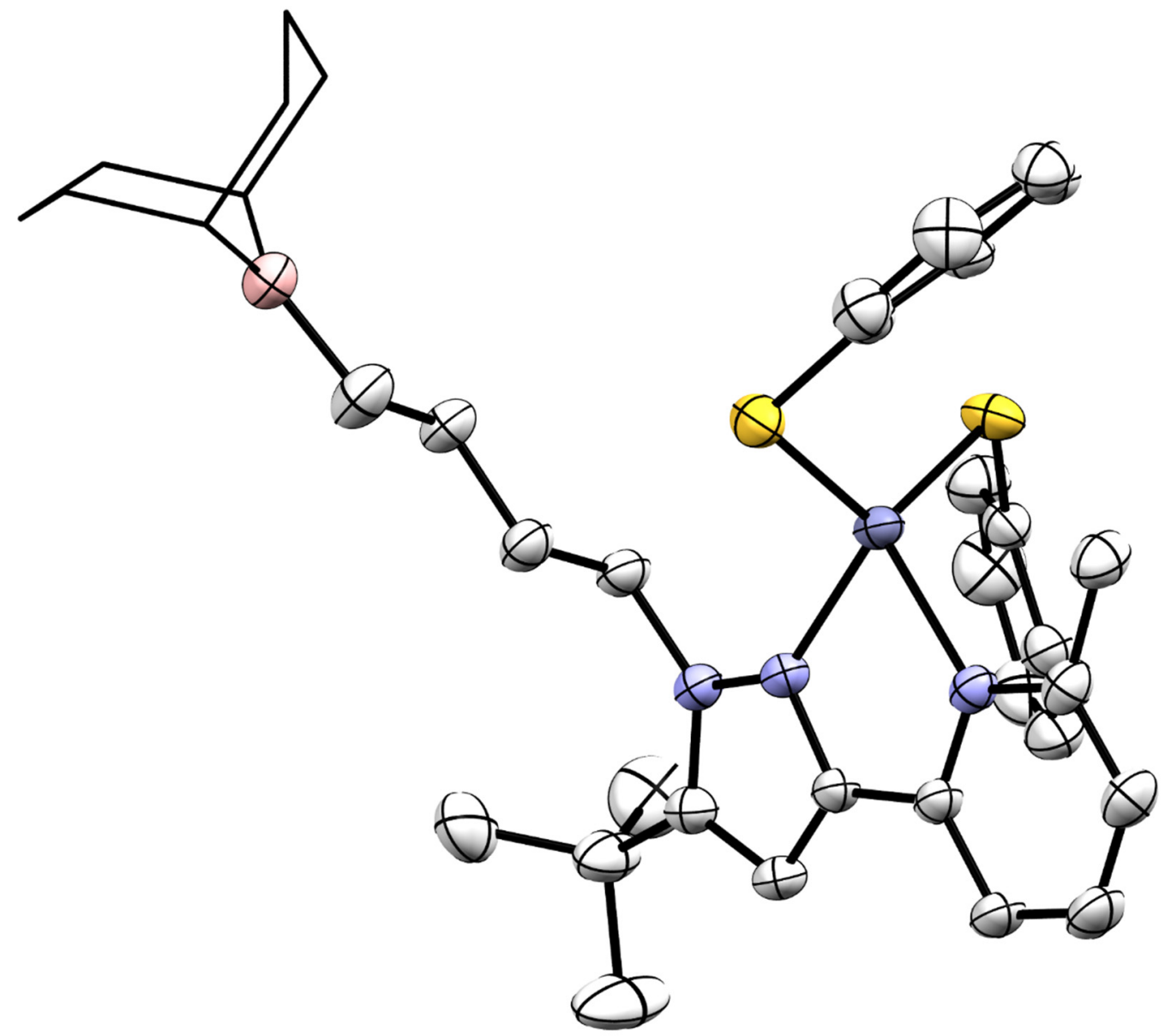

Figure S32 Molecular structure of $\left({ }^{4-B B N} \mathrm{NN}^{\mathrm{BBu}}\right) \mathrm{Zn}(\mathrm{SPh})_{2}$ displayed with $50 \%$ probability ellipsoids.

Hydrogen atoms are omitted for clarity. The 9-BBN substituent is displayed in wireframe for improved clarity. 
Complex: $\left({ }^{3-B P i n} \mathrm{NN}^{\text {tBu }}\right) \mathrm{Zn}(\mathrm{SPh})_{2}$

Local name: JK5275

CCDC: 2073451

Table S6 Experimental parameters for $\left({ }^{3-B P i n} \mathrm{NN}^{\mathrm{BBu}}\right) \mathrm{Zn}(\mathrm{SPh})_{2}$.

\begin{tabular}{|c|c|}
\hline \multicolumn{2}{|l|}{ Crystal data } \\
\hline Chemical formula & $\mathrm{C}_{34} \mathrm{H}_{44} \mathrm{BN}_{3} \mathrm{O}_{2} \mathrm{~S}_{2} \mathrm{Zn}$ \\
\hline$M_{\mathrm{r}}$ & 667.02 \\
\hline Crystal system, space group & Orthorhombic, $P 2_{1} 2_{1} 2_{1}$ \\
\hline Temperature (K) & 150 \\
\hline$a, b, c(\AA)$ & $11.4168(4), 11.7639(5), 26.1088$ (11) \\
\hline$V\left(\AA^{3}\right)$ & $3506.6(2)$ \\
\hline$z$ & 4 \\
\hline Radiation type & Mo $K \alpha$ \\
\hline$\mu\left(\mathrm{mm}^{-1}\right)$ & 0.85 \\
\hline Crystal size $(\mathrm{mm})$ & $0.48 \times 0.33 \times 0.28$ \\
\hline \multicolumn{2}{|l|}{ Data collection } \\
\hline Diffractometer & $\begin{array}{l}\text { Bruker AXS D8 Quest diffractometer with Photonll charge- } \\
\text { integrating pixel array detector (CPAD) }\end{array}$ \\
\hline Absorption correction & $\begin{array}{l}\text { Multi-scan SADABS 2016/2: Krause, L., Herbst-Irmer, R., Sheldrick } \\
\text { G.M. \& Stalke D., J. Appl. Cryst. } 48 \text { (2015) 3-10 }\end{array}$ \\
\hline$T_{\min }, T_{\max }$ & $0.643,0.747$ \\
\hline $\begin{array}{l}\text { No. of measured, independent and } \\
\text { observed }[I>2 s(I)] \text { reflections }\end{array}$ & $75372,13333,11370$ \\
\hline$R_{\text {int }}$ & 0.051 \\
\hline$(\sin \theta / \lambda)_{\max }\left(\AA^{-1}\right)$ & 0.770 \\
\hline \multicolumn{2}{|l|}{ Refinement } \\
\hline$R\left[F^{2}>2 \sigma\left(F^{2}\right)\right], w R\left(F^{2}\right), S$ & $0.029,0.068,1.03$ \\
\hline No. of reflections & 13333 \\
\hline No. of parameters & 461 \\
\hline No. of restraints & 248 \\
\hline $\mathrm{H}$-atom treatment & $\mathrm{H}$-atom parameters constrained \\
\hline$\Delta \rho_{\max }, \Delta \rho_{\min }\left(\mathrm{e} \AA^{-3}\right)$ & $0.34,-0.41$ \\
\hline Absolute structure & $\begin{array}{l}\text { Flack } x \text { determined using } 4449 \text { quotients }[(1+)-(I-)] /[(1+)+(I-)] \\
\text { (Parsons, Flack and Wagner, Acta Cryst. B69 (2013) 249-259). }\end{array}$ \\
\hline Absolute structure parameter & $0.008(3)$ \\
\hline
\end{tabular}

Computer programs: Apex3 v2018.7-2 (Bruker, 2018), SAINT V8.38A (Bruker, 2018), SHELXS97

(Sheldrick, 2008), SHELXL2018/3 (Sheldrick, 2015, 2018), SHELXLE Rev1030 (Hübschle et al., 2011). 


\section{Refinement details:}

The dioxaborolane moiety is disordered by ring-flip. The two disordered moieties were restrained to have similar geometries. $U^{\mathrm{ij}}$ components of ADPs for disordered atoms closer to each other than $2.0 \AA$ were restrained to be similar. Subject to these conditions the occupancy ratio refined to $0.747(3)$ to $0.253(3)$.

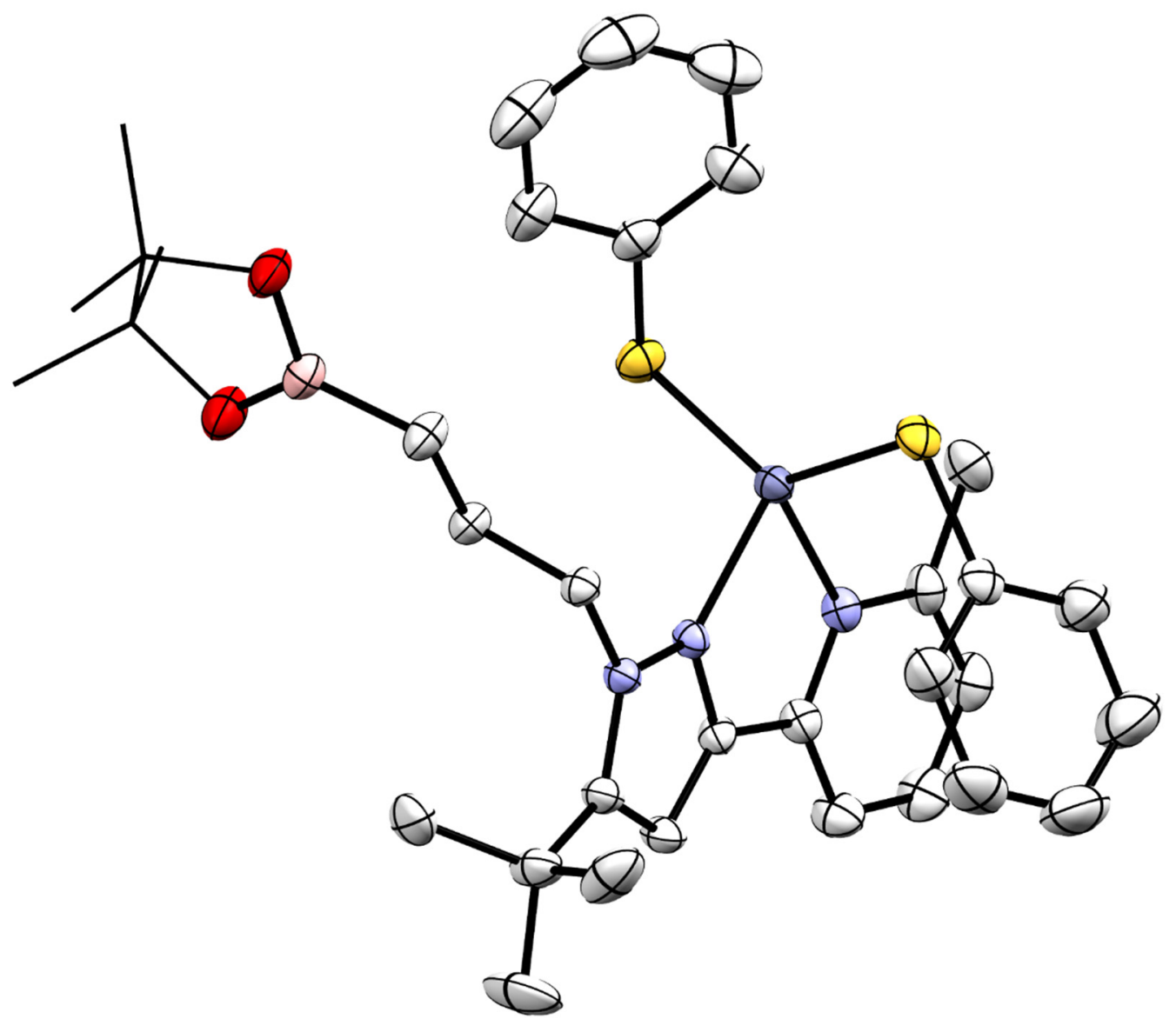

Figure S33 Molecular structure of $\left({ }^{3-B P i n} \mathrm{NN}^{t B u}\right) \mathrm{Zn}(\mathrm{SPh})_{2}$ displayed with $50 \%$ probability ellipsoids. Hydrogen atoms are omitted for clarity. The carbon atoms of the -BPin substituent are displayed in wireframe and disorder is omitted for improved clarity. 
Table S7 Experimentally determined bond distances and angles.

\begin{tabular}{|c|c|c|c|c|}
\hline Compound & $\left({ }^{3-B P i n} N^{t B u}\right) Z n B r_{2}$ & $\left({ }^{3-B P i n} N N^{t B u}\right) Z n(S P h)_{2}$ & $\left({ }^{3-B B N} N N^{t B u}\right) Z n(S P h)_{2}$ & $\left({ }^{4-B B N} N N^{t B u}\right) Z n(S P h)_{2}$ \\
\hline Local name & JK5230 & JK5275 & EN2215 & JK5276 \\
\hline $\mathrm{Zn}-\mathrm{X}$ & $2.3554(3)$ & $2.2596(5)$ & $2.3089(4)$ & $2.2742(4)$ \\
\hline $\mathrm{Zn}-\mathrm{X}$ & $2.3198(3)$ & $2.2693(5)$ & $2.2511(5)$ & $2.2731(4)$ \\
\hline Zn-N $N_{\text {pyridine }}$ & $2.0744(16)$ & $2.1226(15)$ & $2.1029(14)$ & $2.1159(12)$ \\
\hline Zn-N & $2.0378(16)$ & $2.0888(13)$ & $2.0722(13)$ & $2.0680(13)$ \\
\hline B-S & -- & $>5.3$ & $2.1279(18)$ & $>5.6$ \\
\hline Zn-S-C & -- & $103.58(6)$ & $100.84(5)$ & $103.40(5)$ \\
\hline Zn-S-C & -- & $95.00(6)$ & $109.36(5)$ & $103.42(5)$ \\
\hline$\tau_{4}$ & 0.895 & 0.847 & 0.833 & 0.890 \\
\hline$\Sigma \mathrm{B} \alpha$ & $359.9(2)$ & $359.9(4)$ & 316.31(11) & $359.43(17)$ \\
\hline
\end{tabular}




\section{Computational Details}

DFT calculations were performed using the Gaussian 09 revD01 software package ${ }^{10}$ using the B3LYP functional ${ }^{11}$ and an ultrafine integration grid for all atoms. All reported compounds underwent geometry optimization with the $6-31 \mathrm{G}(\mathrm{d})$ basis set, ${ }^{12}$ followed by vibrational frequency calculations. These were used to verify that the structures were truly local energetic minima by the absence of imaginary vibrational modes and to provide entropies of formation at $25^{\circ} \mathrm{C}$. Energies were determined by applying the free energy and enthalpy corrections from the frequency calculations to the scf energies from single point calculations of the optimized geometries with a polarizable continuum model of dichloromethane solvent correction.

In unrestrained geometry optimizations at the B3LYP/6-31G(d) level, the borane optimizes unbound from the sulfur atom regardless of starting B-S distance. To assess the formation of a B-S bond, the geometry optimization was restrained to hold the B-S bond at a set distance of either $2.128 \AA$ or $2.183 \AA$. These values represent the $B-S$ bond distance in the molecular structure of $\left({ }^{3-B B N} N N^{t B u}\right) Z n(S P h)_{2}(2.128 \AA)$ and the longest crystallographically characterized B-S bond containing a four-coordinate boron in the literature $(2.183 \AA$ ) $){ }^{13}$ The values reported at a distance of $2.128 \AA$ are included in the manuscript.

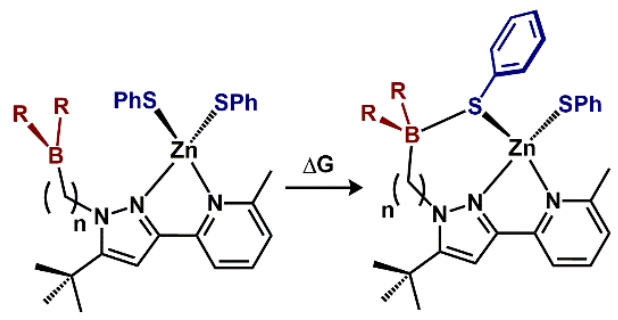

Table S8 Thermodynamics of B-S bond formation (B-S $=2.128 \AA$ ).

\begin{tabular}{|c|c|c|}
\hline Compound & $\Delta \mathrm{G}$ (kcal/mol) & $\Delta \mathrm{H}(\mathrm{kcal} / \mathrm{mol})$ \\
\hline$\left({ }^{3-B B N} N N^{t B u}\right) Z n(S P h)_{2}$ & +103 & +4.3 \\
\hline$\left({ }^{4-B B N} N^{t B u}\right) Z n(S P h)_{2}$ & +17.5 & +12.3 \\
\hline$\left({ }^{3-B P i n} N N^{t B u}\right) Z n(S P h)_{2}$ & +22.4 & +18.8 \\
\hline
\end{tabular}

Table S9 Thermodynamics of B-S bond formation (B-S = 2.183 Å).

\begin{tabular}{|c|c|}
\hline Compound & $\Delta G(\mathrm{kcal} / \mathrm{mol})$ \\
\hline$\left({ }^{3-\mathrm{BBN}} \mathrm{NN}^{\mathrm{tBu}}\right) \mathrm{Zn}(\mathrm{SPh})_{2}$ & +9.9 \\
\hline$\left({ }^{4-B B N} N^{t B u}\right) Z n(S P h)_{2}$ & +16.9 \\
\hline$\left(\left(^{3-B P i n} N^{t B u}\right) Z n(S P h)_{2}\right.$ & +20.9 \\
\hline
\end{tabular}


Table S10 Computationally determined bond distances and angles for compounds that have also been structurally characterized, and experimentally determined bond distances and angles for comparison.

\begin{tabular}{|c|c|c|c|}
\hline Compound & $\left({ }^{3-B P i n} N N^{t B u}\right) Z n(S P h)_{2}$ & $\left({ }^{3-B B N} N^{t B u}\right) Z n(S P h)_{2}$ & $\left({ }^{4-B B N} N N^{t B u}\right) Z n(S P h)_{2}$ \\
\hline \multicolumn{4}{|l|}{ Computation } \\
\hline B-S interaction & none & Restricted to 2.128 & none \\
\hline Zn-S & 2.2843 & 2.2772 & 2.2896 \\
\hline Zn-S & 2.2902 & 2.3533 & 2.2928 \\
\hline$Z n-N_{\text {pyridine }}$ & 2.1339 & 2.1229 & 2.1213 \\
\hline Zn-N $N_{\text {pyrazole }}$ & 2.0889 & 2.0995 & 2.0853 \\
\hline B-S & $>4.9$ & 2.1280 & $>7.2$ \\
\hline Zn-S-C & 103.69 & 107.96 & 105.26 \\
\hline Zn-S-C & 102.25 & 102.25 & 104.26 \\
\hline$\tau_{4}$ & 0.861 & 0.850 & 0.877 \\
\hline$\Sigma \mathrm{B} \alpha$ & 360.0 & 318.19 & 359.68 \\
\hline $\begin{array}{l}\text { Compound } \\
\text { Structure }\end{array}$ & $\left({ }^{3-B P i n} N^{t B u}\right) Z n(S P h)_{2}$ & $\left({ }^{3-B B N} N N^{t B u}\right) Z n(S P h)_{2}$ & $\left({ }^{4-B B N} N N^{t B u}\right) Z n(S P h)_{2}$ \\
\hline Local name & JK5275 & EN2215 & JK5276 \\
\hline Zn-S & $2.2596(5)$ & $2.3089(4)$ & $2.2742(4)$ \\
\hline Zn-S & $2.2693(5)$ & $2.2511(5)$ & $2.2731(4)$ \\
\hline Zn- $N_{\text {pyridine }}$ & $2.1226(15)$ & $2.1029(14)$ & $2.1159(12)$ \\
\hline$Z n-N_{\text {pyrazole }}$ & $2.0888(13)$ & $2.0722(13)$ & $2.0680(13)$ \\
\hline B-S & $>5.3$ & $2.1279(18)$ & $>5.6$ \\
\hline Zn-S-C & $103.58(6)$ & $100.84(5)$ & $103.40(5)$ \\
\hline Zn-S-C & $95.00(6)$ & $109.36(5)$ & $103.42(5)$ \\
\hline$\tau_{4}$ & 0.847 & 0.833 & 0.890 \\
\hline$\Sigma B \alpha$ & $359.9(4)$ & $316.31(11)$ & $359.43(17)$ \\
\hline
\end{tabular}

Table S11 Computationally determined bond distances and angles for all DFT optimized geometries that are not structurally characterized.

\begin{tabular}{|c|c|c|c|}
\hline Compound & $\left({ }^{3-B P i n} N^{t B u}\right) Z n(S P h)_{2}$ & $\left({ }^{3-B B N} N^{t B u}\right) Z n(S P h)_{2}$ & $\left({ }^{4-B B N} N^{t B u}\right) Z n(S P h)_{2}$ \\
\hline B-S interaction & Restricted to 2.128 & none & Restricted to 2.128 \\
\hline Zn-S & 2.2709 & 2.2928 & 2.3275 \\
\hline Zn-S & 2.3328 & 2.2888 & 2.2948 \\
\hline$Z n-N_{\text {pyridine }}$ & 2.1112 & 2.1039 & 2.1094 \\
\hline Zn-N pyrazole & 2.1031 & 2.1020 & 2.1765 \\
\hline B-S & 2.1820 & $>5.0$ & 2.1280 \\
\hline Zn-S-C & 105.82 & 104.63 & 104.73 \\
\hline Zn-S-C & 105.37 & 102.47 & 110.05 \\
\hline$\tau_{4}$ & 0.867 & 0.889 & 0.852 \\
\hline$\Sigma B \alpha$ & 314.05 & 359.93 & 317.211 \\
\hline
\end{tabular}



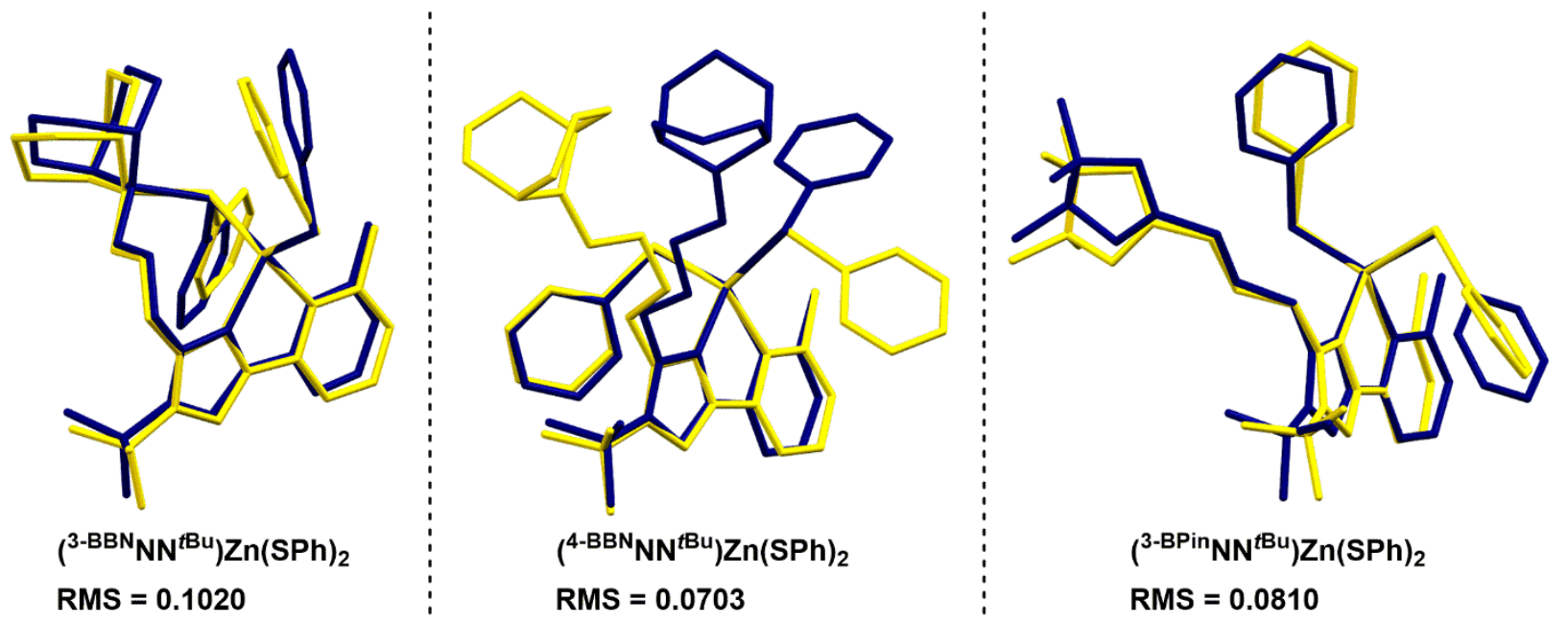

Figure S34 Structural overlay of DFT optimized geometries and molecular structures. Molecular structure is in blue, with the DFT optimized geometries in maize. Overlay similarities (RMS) are in included. Hydrogen atoms are omitted for clarity.

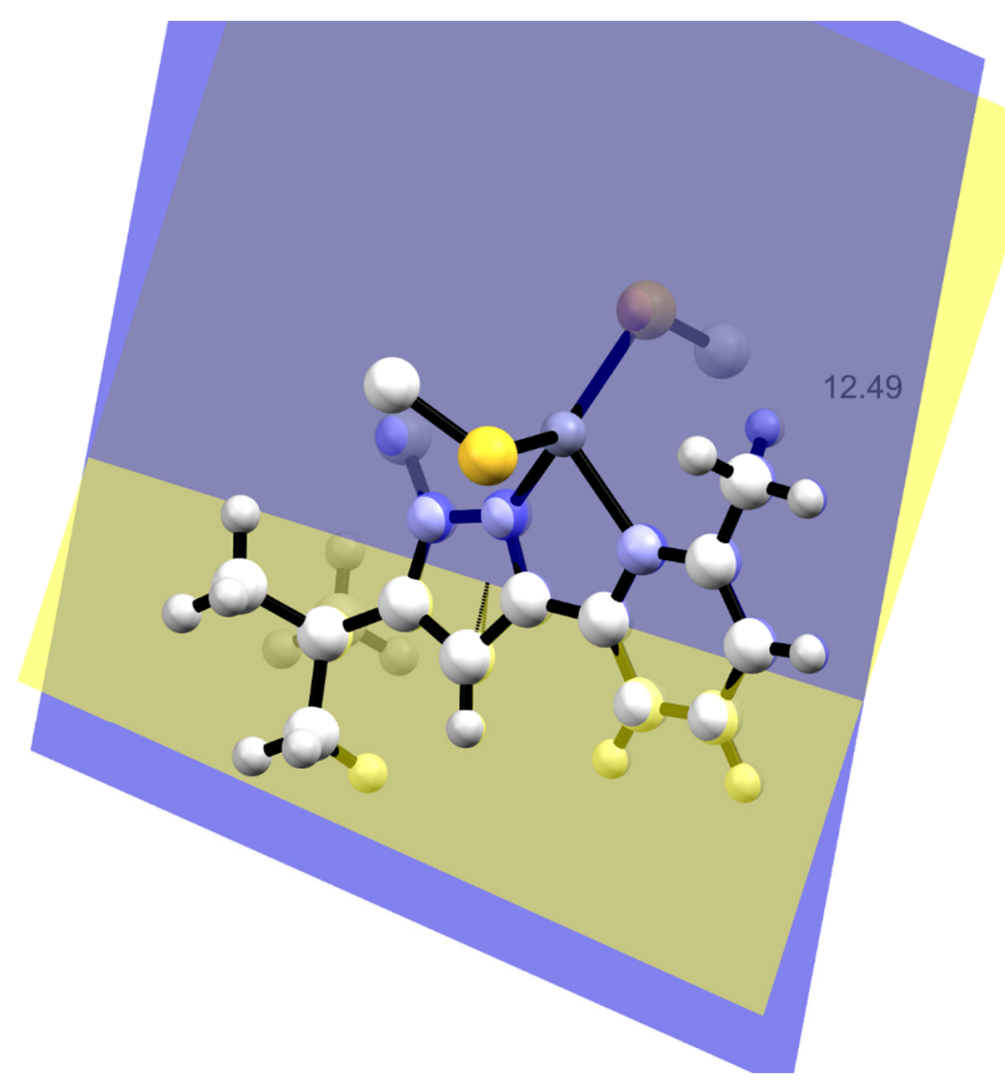

Figure S35 DFT optimized structure of $\left({ }^{4-B B N} \mathrm{NN}^{\mathrm{tBu}}\right) \mathrm{Zn}(\mathrm{SPh})_{2}(\mathrm{~B}-\mathrm{S}=2.128 \AA$ ) with pyrazole (maize) and pyridine (blue) planes displayed to highlight the ligand torsion angle $\left(12.49^{\circ}\right)$. Multiple portions of the molecule have been omitted to improve clarity. 


\begin{tabular}{lll} 
Orientation & Angle $\left({ }^{\circ}\right)$ & Approx. Energy \\
\hline Eclipsed (C-C) & 0 (or 360$)$ & $6.6 \mathrm{kcal} / \mathrm{mol}$ \\
Gauche & 60 (or 300$)$ & $0.6 \mathrm{kcal} / \mathrm{mol}$ \\
Eclipsed (C-H) & 120 (or 240$)$ & $3.4 \mathrm{kcal} / \mathrm{mol}$ \\
Staggered & 180 & $0.0 \mathrm{kcal} / \mathrm{mol}$
\end{tabular}

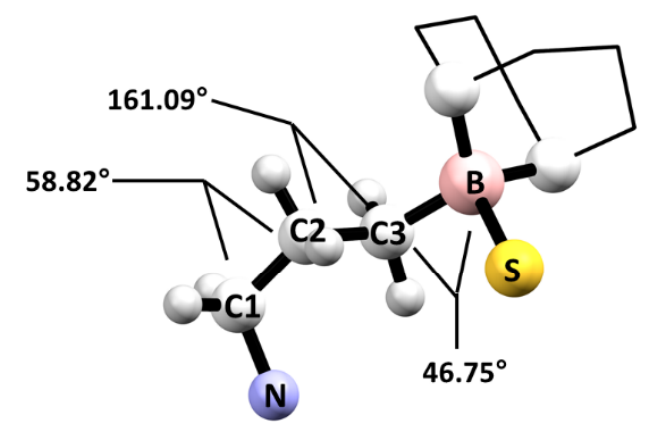

\begin{tabular}{lll} 
Dihedral & Angle $\left(^{\circ}\right)$ & Orientation \\
\hline N-C1-C2-C3 & 58.82 & Gauche \\
C1-C2-C3-B & 161.09 & Staggered \\
C2-C3-B-S & 46.75 & Gauche \\
\hline
\end{tabular}

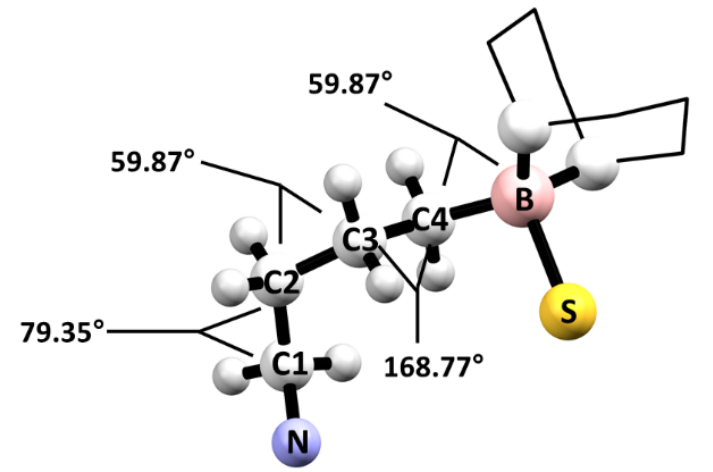

\begin{tabular}{lll} 
Dihedral & Angle $\left(^{\circ}\right)$ & Orientation \\
\hline N-C1-C2-C3 & 79.35 & Gauche \\
C1-C2-C3-C4 & 59.87 & Gauche \\
C2-C3-C4-B & 168.77 & Staggered \\
C3-C4-B-S & 46.75 & Gauche \\
\hline
\end{tabular}

Figure S36 Comparison of the alkyl tether of the DFT optimized structures of $\left({ }^{3-B B N} \mathrm{NN}^{\mathrm{tBu}}\right) \mathrm{Zn}(\mathrm{SPh})_{2}$ and $\left({ }^{4-}\right.$ $\left.{ }^{\mathrm{BBN}} \mathrm{NN}^{\mathrm{tBu}}\right) \mathrm{Zn}(\mathrm{SPh})_{2}(\mathrm{~B}-\mathrm{S}=2.128 \AA$ ). For reference, the table at the top are approximate values associated with each orientation. ${ }^{14}$
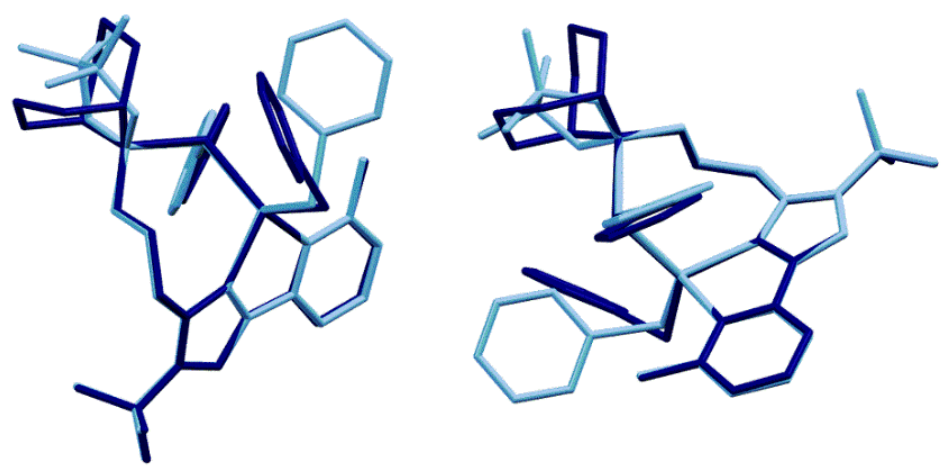

Figure S37 Two overlays of the DFT optimized geometries for $\left({ }^{3-B B N} \mathrm{NN}^{\mathrm{tBu}}\right) \mathrm{Zn}(\mathrm{SPh})_{2}$ (dark blue) and $\left(^{3-}\right.$ $\left.{ }^{B P i n} N_{N}{ }^{t B u}\right) Z n(S P h)_{2}$ (light blue) with B-S bond lengths of $2.128 \AA$. Hydrogen atoms are omitted for clarity. 

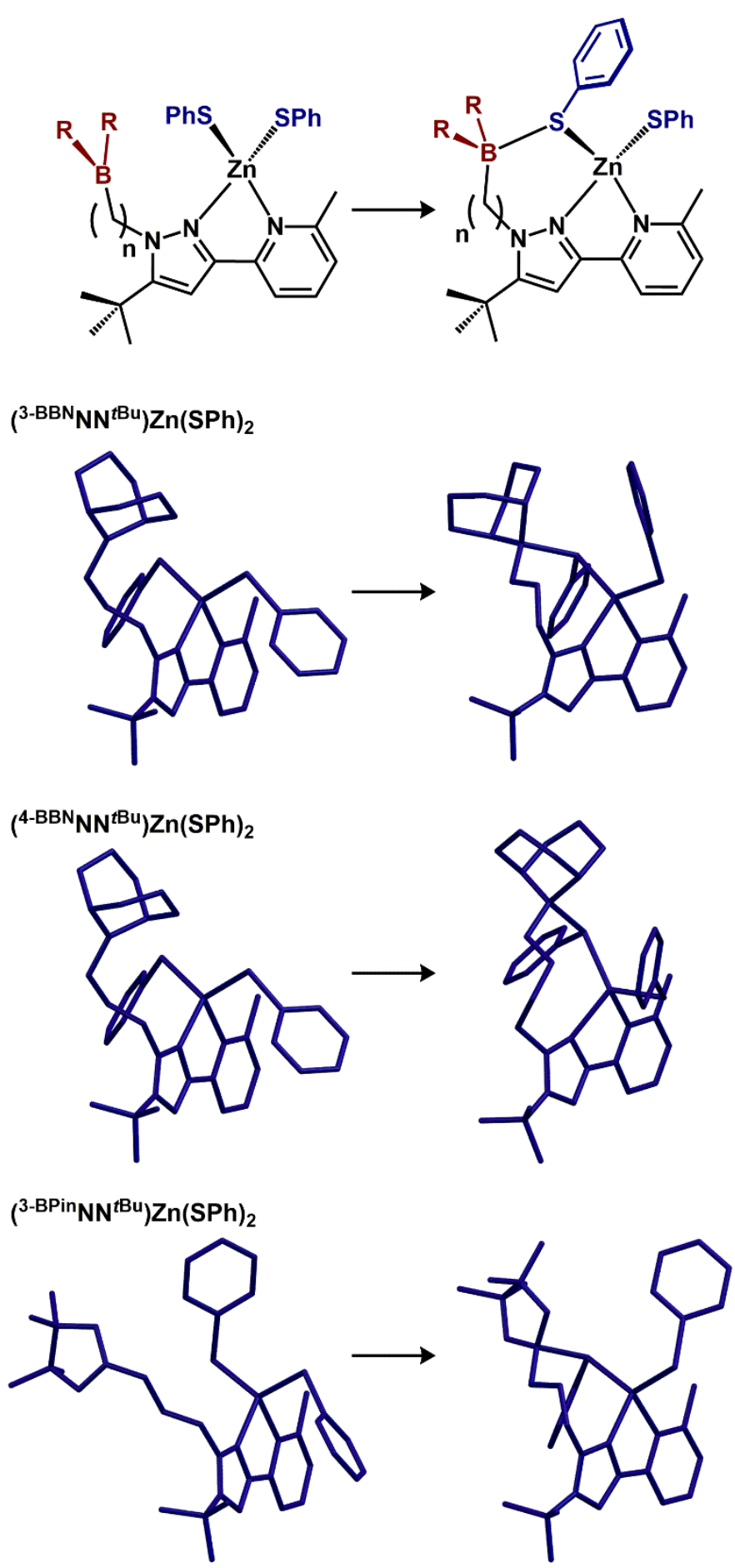

Figure S38 All DFT optimized geometries. Complexes on the right contain B-S bonds of $2.128 \AA$. Hydrogen atoms are omitted for clarity. 


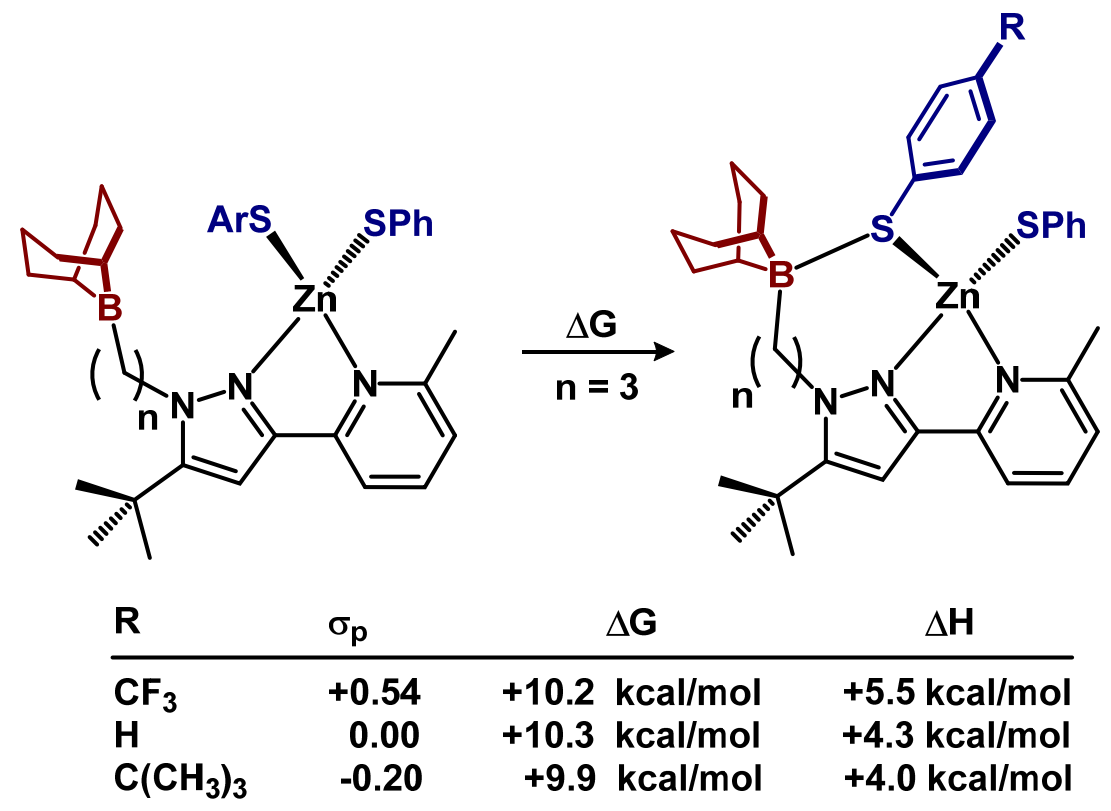

Figure S39 Result of para-substitution on B-S binding.
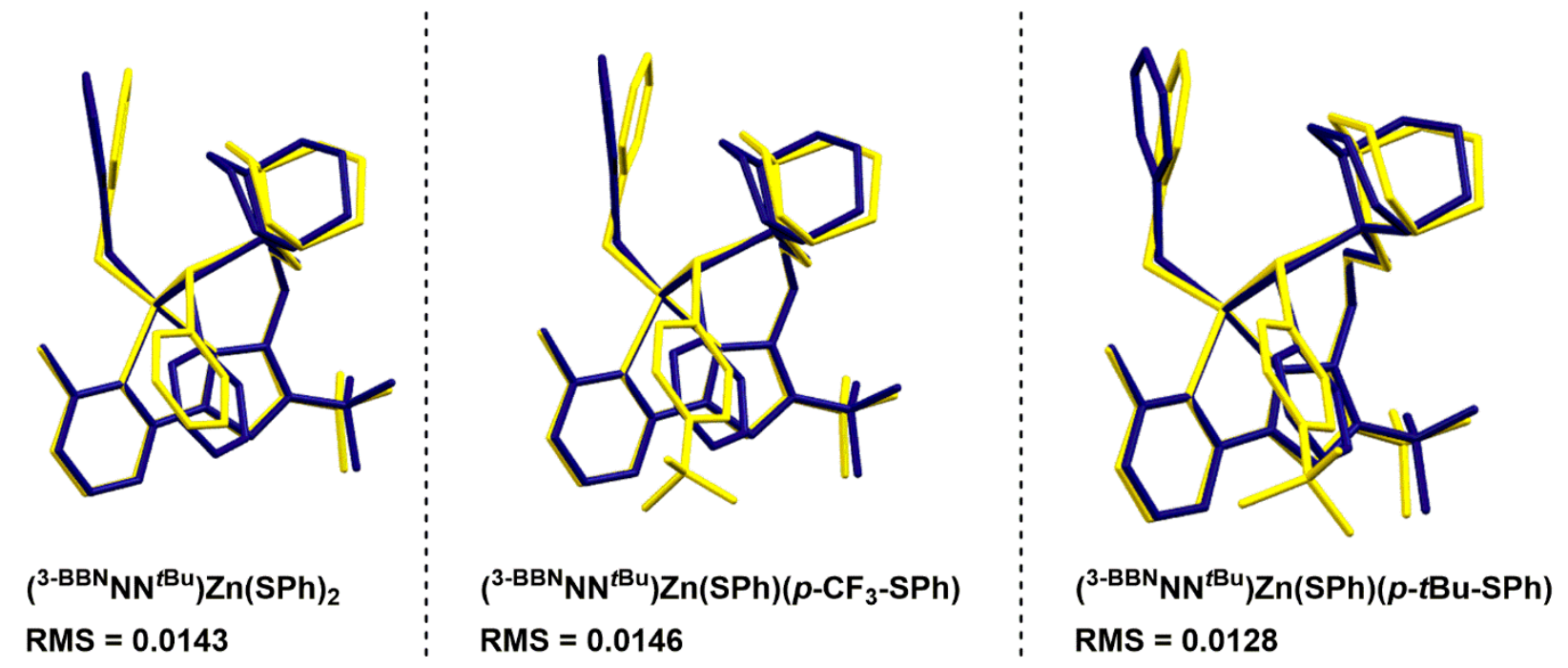

Figure S40 Structural overlay of para-substituted SPh DFT optimized geometries and molecular structure of $\left({ }^{3-B B N} \mathrm{NN}^{t \mathrm{Bu}}\right) \mathrm{Zn}(\mathrm{SPh})_{2}(\mathbf{B}-3)$. Molecular structure of B-3 is in blue, with the DFT optimized geometries in maize. Overlay similarities (RMS) are in included, with overlay restriction only at the Pyridine- $\mathrm{N}$, pyrazole$\mathrm{N}$, and $\mathrm{Zn}$ to show similar primary coordination spheres. Hydrogen atoms are omitted for clarity. 


\section{Optimized Coordinates}

$\left.{ }^{3-B B N} \mathrm{NN}^{\text {tBu }}\right) \mathrm{Zn}(\mathrm{SPh})_{2}$ (No B-S interaction)

\begin{tabular}{|c|c|c|c|}
\hline $\mathrm{Zn}$ & 9.12194800 & 7.64525100 & 8.92894900 \\
\hline$S$ & 8.22732200 & 9.33100200 & 10.19972900 \\
\hline$S$ & 8.56100000 & 5.48919600 & 9.45360900 \\
\hline $\mathrm{N}$ & 11.15794900 & 7.96237900 & 8.50397200 \\
\hline $\mathrm{N}$ & 9.06848500 & 7.92118900 & 6.84583800 \\
\hline $\mathrm{N}$ & 8.18236000 & 7.83478300 & 5.83709000 \\
\hline C & 11.76021400 & 7.75994800 & 10.85038400 \\
\hline C & 12.14533400 & 8.00011000 & 9.41880100 \\
\hline C & 13.46611600 & 8.25187200 & 9.02917000 \\
\hline C & 13.75697600 & 8.46576700 & 7.68483600 \\
\hline C & 12.72876400 & 8.42379800 & 6.74715600 \\
\hline C & 11.43286200 & 8.16735200 & 7.19471200 \\
\hline C & 10.27611800 & 8.09144800 & 6.29649600 \\
\hline C & 10.15945600 & 8.12965500 & 4.89765400 \\
\hline C & 8.80587300 & 7.96096200 & 4.61876900 \\
\hline C & 8.13560300 & 7.88070600 & 3.25065300 \\
\hline C & 7.56374400 & 6.46171900 & 3.00732100 \\
\hline C & 9.20070900 & 8.14920600 & 2.16455200 \\
\hline C & 7.02713700 & 8.94926200 & 3.09081500 \\
\hline C & 6.76268400 & 7.68401100 & 6.19655400 \\
\hline C & 6.13248200 & 8.98388200 & 6.71770500 \\
\hline C & 4.60449100 & 8.86226600 & 6.85893500 \\
\hline C & 2.39439800 & 7.69173300 & 8.00227700 \\
\hline C & 2.14244900 & 6.16145900 & 7.85591500 \\
\hline C & 2.92882800 & 5.26976300 & 8.83987900 \\
\hline C & 4.41007400 & 5.66822700 & 9.00429000 \\
\hline C & 4.67597300 & 7.19816500 & 9.15806200 \\
\hline C & 4.12669600 & 7.81524800 & 10.48222400 \\
\hline C & 2.58896500 & 7.85176100 & 10.61001500 \\
\hline C & 1.86002600 & 8.31088100 & 9.32907200 \\
\hline C & 9.24718700 & 10.75878600 & 9.83998700 \\
\hline C & 9.84434500 & 11.45911700 & 10.90378400 \\
\hline C & 10.60946400 & 12.60306700 & 10.67128900 \\
\hline C & 10.80268200 & 13.07434400 & 9.37099000 \\
\hline C & 10.21136700 & 12.39075500 & 8.30613000 \\
\hline C & 9.43823200 & 11.25159800 & 8.53622000 \\
\hline C & 9.80575200 & 4.54108200 & 8.57712400 \\
\hline C & 9.77834400 & 4.38071800 & 7.18021200 \\
\hline C & 10.73405400 & 3.59880600 & 6.52866000 \\
\hline C & 11.73445000 & 2.95015000 & 7.25581900 \\
\hline C & 11.76594200 & 3.09161800 & 8.64491300 \\
\hline
\end{tabular}




\begin{tabular}{cccc}
$\mathrm{C}$ & 10.81478900 & 3.87738500 & 9.29762700 \\
$\mathrm{~B}$ & 3.95741700 & 7.92951400 & 7.96254400 \\
$\mathrm{H}$ & 8.96434400 & 10.74404800 & 7.70104500 \\
$\mathrm{H}$ & 10.34243700 & 12.75147200 & 7.28812300 \\
$\mathrm{H}$ & 11.39959200 & 13.96445300 & 9.19046100 \\
$\mathrm{H}$ & 11.05762100 & 13.12704400 & 11.51251700 \\
$\mathrm{H}$ & 9.69650300 & 11.09915100 & 11.91805300 \\
$\mathrm{H}$ & 10.83813800 & 3.97553400 & 10.37927900 \\
$\mathrm{H}$ & 12.53364500 & 2.58615500 & 9.22645600 \\
$\mathrm{H}$ & 12.47396700 & 2.33659600 & 6.74793000 \\
$\mathrm{H}$ & 10.68936200 & 3.48839300 & 5.44724900 \\
$\mathrm{H}$ & 8.99048100 & 4.86152300 & 6.60744400 \\
$\mathrm{H}$ & 5.76449900 & 7.33528000 & 9.17866200 \\
$\mathrm{H}$ & 1.86395800 & 8.18908400 & 7.17591800 \\
$\mathrm{H}$ & 1.95200400 & 9.40465500 & 9.24674700 \\
$\mathrm{H}$ & 0.78264100 & 8.10960700 & 9.42762500 \\
$\mathrm{H}$ & 2.21836000 & 6.86713700 & 10.90982800 \\
$\mathrm{H}$ & 2.31734900 & 8.52738200 & 11.43253200 \\
$\mathrm{H}$ & 4.55231500 & 7.27523200 & 11.33983600 \\
$\mathrm{H}$ & 4.51614000 & 8.84044400 & 10.55704500 \\
$\mathrm{H}$ & 2.43464600 & 5.27470200 & 9.81542100 \\
$\mathrm{H}$ & 2.87703400 & 4.22839800 & 8.49353900 \\
$\mathrm{H}$ & 6.23878500 & 7.32806700 & 5.31010400 \\
$\mathrm{H}$ & 4.84652800 & 5.13312900 & 9.85855800 \\
$\mathrm{H}$ & 4.97013400 & 5.31295600 & 8.12625000 \\
$\mathrm{H}$ & 1.06661400 & 5.94880700 & 7.94651100 \\
$\mathrm{H}$ & 2.41553900 & 5.87381900 & 6.82849300 \\
$\mathrm{H}$ & 12.63627900 & 7.77632500 & 11.50395400 \\
$\mathrm{H}$ & 11.05000700 & 8.52435300 & 11.18621800 \\
$\mathrm{H}$ & 11.25814500 & 6.79088500 & 10.95213200 \\
$\mathrm{H}$ & 8.35467200 & 5.70733400 & 3.07886200 \\
$\mathrm{H}$ & 6.77909500 & 6.19036400 & 3.71949500 \\
$\mathrm{H}$ & 7.13050000 & 6.40574700 & 2.00207200 \\
$\mathrm{H}$ & 7.42562500 & 9.95487500 & 3.26459700 \\
$\mathrm{H}$ & 6.63195600 & 8.91479500 & 2.06919500 \\
$\mathrm{H}$ & 6.18567600 & 8.79835500 & 3.77140000 \\
$\mathrm{H}$ & 10.00138200 & 7.40246900 & 2.19047400 \\
$\mathrm{H}$ & 8.73279200 & 8.10027800 & 1.17579300 \\
$\mathrm{H}$ & 9.64791200 & 9.14300700 & 2.27550000 \\
\hline & 10.95255200 & 8.24378200 & 4.17639000 \\
$\mathrm{H}$ & 14.92179300 & 8.58809800 & 5.69281400 \\
$\mathrm{H}$ & 14.24836400 & 8.27722500 & 9.78015200 \\
$\mathrm{H}$ & 6.89305900 & 6.95130400
\end{tabular}




$\begin{array}{llll}\mathrm{H} & 6.58642300 & 9.23895800 & 7.68194300 \\ \mathrm{H} & 6.38474800 & 9.79479400 & 6.02112900 \\ \mathrm{H} & 4.20290200 & 9.86300100 & 7.10284900 \\ \mathrm{H} & 4.14612600 & 8.64107300 & 5.87962600\end{array}$

\begin{tabular}{|c|c|c|c|}
\hline \multicolumn{4}{|c|}{$\left({ }^{3-\mathrm{BBN}} \mathrm{NN}^{t \mathrm{Bu}}\right) \mathrm{Zn}(\mathrm{SPh})_{2}(\mathrm{~B}-\mathrm{S}=2.128 \AA \AA$} \\
\hline $\mathrm{Zn}$ & 10.45545400 & 7.40661800 & 8.23917100 \\
\hline S & 9.97322700 & 8.75572900 & 10.10610800 \\
\hline S & 10.98583700 & 5.19207600 & 8.25670700 \\
\hline $\mathrm{N}$ & 11.92187500 & 8.33040000 & 7.01316700 \\
\hline $\mathrm{N}$ & 9.30157400 & 7.95855500 & 6.57426800 \\
\hline $\mathrm{N}$ & 8.02203700 & 7.91496100 & 6.16348800 \\
\hline C & 13.71032800 & 7.91869300 & 8.61845200 \\
\hline C & 13.23671600 & 8.43860500 & 7.29082800 \\
\hline C & 14.11399300 & 9.02125100 & 6.36838700 \\
\hline C & 13.62243100 & 9.49463000 & 5.15545200 \\
\hline C & 12.26400800 & 9.37671100 & 4.87680300 \\
\hline C & 11.43752900 & 8.78447300 & 5.83240400 \\
\hline C & 9.99813200 & 8.59791000 & 5.62669500 \\
\hline C & 9.14245200 & 8.97110800 & 4.57905200 \\
\hline C & 7.87528200 & 8.52168500 & 4.94062400 \\
\hline C & 6.57273800 & 8.67134600 & 4.16046800 \\
\hline C & 5.93378800 & 7.29413800 & 3.85658100 \\
\hline C & 6.88470600 & 9.34669300 & 2.80657000 \\
\hline C & 5.57878000 & 9.58482600 & 4.92020700 \\
\hline C & 7.04519500 & 7.16435100 & 6.98147600 \\
\hline C & 7.26494500 & 7.33133700 & 8.48673400 \\
\hline C & 7.19827300 & 8.77396400 & 9.01198700 \\
\hline C & 7.66465100 & 10.48067600 & 11.13466000 \\
\hline C & 6.13364500 & 10.62782700 & 11.37278300 \\
\hline C & 5.47571000 & 9.48622300 & 12.18515400 \\
\hline C & 5.97733500 & 8.06267000 & 11.84430300 \\
\hline C & 7.51351900 & 7.92803200 & 11.62855900 \\
\hline C & 8.32282900 & 8.13168700 & 12.93296700 \\
\hline C & 8.32380000 & 9.56945800 & 13.49775800 \\
\hline C & 8.48391600 & 10.67884700 & 12.43475400 \\
\hline C & 10.70453700 & 10.33155300 & 9.63751300 \\
\hline C & 11.76872000 & 10.82249900 & 10.41016600 \\
\hline C & 12.37240300 & 12.04162600 & 10.09578800 \\
\hline C & 11.91789900 & 12.79208800 & 9.01006100 \\
\hline C & 10.85519200 & 12.31326300 & 8.24103200 \\
\hline C & 10.25174700 & 11.09210100 & 8.54884900 \\
\hline C & 10.13181300 & 4.42993000 & 9.63693300 \\
\hline
\end{tabular}




\begin{tabular}{|c|c|c|c|}
\hline C & 9.44739900 & 3.22264000 & 9.41944900 \\
\hline C & 8.81659300 & 2.56157800 & 10.47424000 \\
\hline C & 8.84906400 & 3.09547000 & 11.76411800 \\
\hline C & 9.52772700 & 4.29508800 & 11.98895300 \\
\hline C & 10.17049500 & 4.95388100 & 10.93918600 \\
\hline B & 7.89338300 & 9.01248700 & 10.47585200 \\
\hline $\mathrm{H}$ & 9.42459300 & 10.73164700 & 7.94697600 \\
\hline $\mathrm{H}$ & 10.48792100 & 12.89417000 & 7.39851900 \\
\hline $\mathrm{H}$ & 12.38326300 & 13.74392200 & 8.76856900 \\
\hline $\mathrm{H}$ & 13.19371400 & 12.40714300 & 10.70692000 \\
\hline $\mathrm{H}$ & 12.11099900 & 10.24698000 & 11.26535800 \\
\hline $\mathrm{H}$ & 10.70598500 & 5.87881200 & 11.13165300 \\
\hline $\mathrm{H}$ & 9.56221300 & 4.72472800 & 12.98692200 \\
\hline $\mathrm{H}$ & 8.35245300 & 2.58349900 & 12.58402400 \\
\hline $\mathrm{H}$ & 8.29310200 & 1.62742000 & 10.28379700 \\
\hline $\mathrm{H}$ & 9.41448400 & 2.80731300 & 8.41627600 \\
\hline $\mathrm{H}$ & 7.68805100 & 6.88720900 & 11.31415600 \\
\hline $\mathrm{H}$ & 7.94791600 & 11.29803600 & 10.45205700 \\
\hline $\mathrm{H}$ & 9.54506900 & 10.75173000 & 12.16366000 \\
\hline $\mathrm{H}$ & 8.23170300 & 11.64687600 & 12.89733400 \\
\hline $\mathrm{H}$ & 7.40371300 & 9.73924200 & 14.06692100 \\
\hline $\mathrm{H}$ & 9.13513300 & 9.66267500 & 14.23451700 \\
\hline $\mathrm{H}$ & 7.96031200 & 7.44672200 & 13.71658400 \\
\hline $\mathrm{H}$ & 9.36183400 & 7.83702700 & 12.73476000 \\
\hline $\mathrm{H}$ & 5.62454600 & 9.67262600 & 13.25312100 \\
\hline $\mathrm{H}$ & 4.38644300 & 9.52552800 & 12.03796400 \\
\hline $\mathrm{H}$ & 5.64693200 & 7.37589000 & 12.63962400 \\
\hline$H$ & 5.46880600 & 7.72043100 & 10.93131100 \\
\hline $\mathrm{H}$ & 5.92232800 & 11.58337000 & 11.87877700 \\
\hline $\mathrm{H}$ & 5.63739500 & 10.69770600 & 10.39517600 \\
\hline $\mathrm{H}$ & 14.79846600 & 7.98396000 & 8.70003400 \\
\hline $\mathrm{H}$ & 13.26768600 & 8.50394700 & 9.43290300 \\
\hline $\mathrm{H}$ & 13.40263000 & 6.87563900 & 8.75625900 \\
\hline $\mathrm{H}$ & 6.63513200 & 6.64982700 & 3.31495200 \\
\hline $\mathrm{H}$ & 5.61453100 & 6.76279600 & 4.75611800 \\
\hline $\mathrm{H}$ & 5.04841400 & 7.43476100 & 3.22618700 \\
\hline $\mathrm{H}$ & 6.01385600 & 10.57618600 & 5.08656900 \\
\hline $\mathrm{H}$ & 4.66631600 & 9.70926200 & 4.32591800 \\
\hline $\mathrm{H}$ & 5.28641400 & 9.18536600 & 5.89503000 \\
\hline $\mathrm{H}$ & 7.57716400 & 8.74715400 & 2.20561700 \\
\hline $\mathrm{H}$ & 5.95746600 & 9.45999900 & 2.23527700 \\
\hline $\mathrm{H}$ & 7.31509300 & 10.34480900 & 2.94187400 \\
\hline $\mathrm{H}$ & 9.40059000 & 9.50341000 & 3.67785700 \\
\hline $\mathrm{H}$ & 11.84972000 & 9.72895300 & 3.93875800 \\
\hline
\end{tabular}




$\begin{array}{cccc}\mathrm{H} & 14.29212800 & 9.94927600 & 4.43107600 \\ \mathrm{H} & 15.16837400 & 9.09776300 & 6.61091500 \\ \mathrm{H} & 6.05846200 & 7.53240800 & 6.70616900 \\ \mathrm{H} & 7.11361100 & 6.10932100 & 6.69005400 \\ \mathrm{H} & 6.50093800 & 6.71343100 & 8.97464900 \\ \mathrm{H} & 8.20992200 & 6.84403800 & 8.75405600 \\ \mathrm{H} & 7.64458600 & 9.45715100 & 8.27519700 \\ \mathrm{H} & 6.13813600 & 9.06454400 & 9.05640100\end{array}$

\begin{tabular}{|c|c|c|c|}
\hline \multicolumn{4}{|c|}{$\left({ }^{3-B B N} N^{t B u}\right) Z n(S P h)_{2}(B-S=2.183 \AA)$} \\
\hline $\mathrm{Zn}$ & 10.45878500 & 7.40713800 & 8.24166800 \\
\hline S & 9.98082900 & 8.74816600 & 10.11142900 \\
\hline$S$ & 10.98275400 & 5.19066400 & 8.25412400 \\
\hline $\mathrm{N}$ & 11.91949600 & 8.33317100 & 7.01263100 \\
\hline $\mathrm{N}$ & 9.29902500 & 7.95615400 & 6.57738400 \\
\hline $\mathrm{N}$ & 8.01942800 & 7.90844000 & 6.16693300 \\
\hline C & 13.71077300 & 7.92385100 & 8.61515900 \\
\hline C & 13.23421100 & 8.44527400 & 7.28917900 \\
\hline C & 14.10838400 & 9.03330400 & 6.36720400 \\
\hline C & 13.61370700 & 9.50805700 & 5.15606800 \\
\hline C & 12.25535600 & 9.38619700 & 4.87870100 \\
\hline C & 11.43206800 & 8.78864100 & 5.83374800 \\
\hline C & 9.99296200 & 8.59790700 & 5.62950500 \\
\hline C & 9.13556300 & 8.96885800 & 4.58240600 \\
\hline C & 7.87008900 & 8.51517000 & 4.94430300 \\
\hline C & 6.56672500 & 8.66144400 & 4.16483900 \\
\hline C & 5.93328700 & 7.28242700 & 3.85747600 \\
\hline C & 6.87560900 & 9.34144100 & 2.81253700 \\
\hline C & 5.56932200 & 9.56915800 & 4.92695400 \\
\hline C & 7.04578400 & 7.15284100 & 6.98390600 \\
\hline C & 7.25812200 & 7.32632900 & 8.48952100 \\
\hline C & 7.18266600 & 8.77040400 & 9.00820000 \\
\hline C & 7.62944500 & 10.48667700 & 11.12729000 \\
\hline C & 6.09718600 & 10.63800000 & 11.36167400 \\
\hline C & 5.43627200 & 9.50220200 & 12.17978100 \\
\hline C & 5.93396700 & 8.07585200 & 11.84499400 \\
\hline C & 7.47139400 & 7.93628900 & 11.63406200 \\
\hline C & 8.27912800 & 8.14505600 & 12.93845200 \\
\hline C & 8.28301800 & 9.58574600 & 13.49574200 \\
\hline C & 8.44756100 & 10.68910400 & 12.42719400 \\
\hline C & 10.71130800 & 10.32667600 & 9.65443400 \\
\hline C & 11.76512000 & 10.82192400 & 10.43894800 \\
\hline C & 12.36509700 & 12.04519100 & 10.13392100 \\
\hline
\end{tabular}




\begin{tabular}{|c|c|c|c|}
\hline C & 11.91814900 & 12.79594000 & 9.04519000 \\
\hline C & 10.86609100 & 12.31312400 & 8.26414400 \\
\hline C & 10.26581000 & 11.08822700 & 8.56318700 \\
\hline C & 10.12391600 & 4.42922600 & 9.63162100 \\
\hline C & 9.44545900 & 3.21867200 & 9.41324000 \\
\hline C & 8.81012300 & 2.55824300 & 10.46568700 \\
\hline C & 8.83191500 & 3.09590500 & 11.75423200 \\
\hline C & 9.50461400 & 4.29872500 & 11.98000700 \\
\hline C & 10.15192900 & 4.95701400 & 10.93268300 \\
\hline B & 7.84605800 & 9.01553600 & 10.48123500 \\
\hline $\mathrm{H}$ & 9.44623000 & 10.72532800 & 7.95225200 \\
\hline $\mathrm{H}$ & 10.50437900 & 12.89396500 & 7.41915200 \\
\hline $\mathrm{H}$ & 12.38099300 & 13.75074900 & 8.81070700 \\
\hline $\mathrm{H}$ & 13.17822500 & 12.41343100 & 10.75433100 \\
\hline $\mathrm{H}$ & 12.10246700 & 10.24567800 & 11.29560400 \\
\hline $\mathrm{H}$ & 10.68293800 & 5.88429600 & 11.12628500 \\
\hline $\mathrm{H}$ & 9.53124700 & 4.73113600 & 12.97704400 \\
\hline $\mathrm{H}$ & 8.33189400 & 2.58435400 & 12.57232900 \\
\hline $\mathrm{H}$ & 8.29151300 & 1.62152600 & 10.27440700 \\
\hline $\mathrm{H}$ & 9.42067500 & 2.80036200 & 8.41106200 \\
\hline $\mathrm{H}$ & 7.64444900 & 6.89440400 & 11.32306700 \\
\hline $\mathrm{H}$ & 7.91586200 & 11.29804600 & 10.43912900 \\
\hline $\mathrm{H}$ & 9.50883900 & 10.75660100 & 12.15592300 \\
\hline $\mathrm{H}$ & 8.19801900 & 11.66039800 & 12.88421400 \\
\hline $\mathrm{H}$ & 7.36297500 & 9.76095400 & 14.06335800 \\
\hline $\mathrm{H}$ & 9.09403400 & 9.68050400 & 14.23256700 \\
\hline $\mathrm{H}$ & 7.91414000 & 7.46488800 & 13.72504100 \\
\hline $\mathrm{H}$ & 9.31745300 & 7.84719200 & 12.74213700 \\
\hline $\mathrm{H}$ & 5.58589900 & 9.69305500 & 13.24678200 \\
\hline $\mathrm{H}$ & 4.34716700 & 9.54378800 & 12.03235400 \\
\hline $\mathrm{H}$ & 5.59969400 & 7.39226600 & 12.64130600 \\
\hline $\mathrm{H}$ & 5.42761200 & 7.73254900 & 10.93108800 \\
\hline $\mathrm{H}$ & 5.88698900 & 11.59710500 & 11.86113700 \\
\hline $\mathrm{H}$ & 5.60285600 & 10.70226100 & 10.38258600 \\
\hline $\mathrm{H}$ & 14.79885200 & 7.99163000 & 8.69549700 \\
\hline $\mathrm{H}$ & 13.26756800 & 8.50622500 & 9.43138300 \\
\hline $\mathrm{H}$ & 13.40591200 & 6.87974700 & 8.75130600 \\
\hline $\mathrm{H}$ & 6.63713600 & 6.64251200 & 3.31390000 \\
\hline $\mathrm{H}$ & 5.61655500 & 6.74720800 & 4.75561800 \\
\hline $\mathrm{H}$ & 5.04712400 & 7.42108100 & 3.22774900 \\
\hline $\mathrm{H}$ & 6.00055200 & 10.56182700 & 5.09551900 \\
\hline $\mathrm{H}$ & 4.65620400 & 9.69139600 & 4.33320600 \\
\hline $\mathrm{H}$ & 5.27879100 & 9.16633100 & 5.90093600 \\
\hline $\mathrm{H}$ & 7.56989300 & 8.74595700 & 2.20967100 \\
\hline
\end{tabular}




$\begin{array}{cccc}\mathrm{H} & 5.94768100 & 9.45290400 & 2.24199900 \\ \mathrm{H} & 7.30250600 & 10.34072000 & 2.95027800 \\ \mathrm{H} & 9.39161300 & 9.50232200 & 3.68130400 \\ \mathrm{H} & 11.83875000 & 9.73962500 & 3.94212800 \\ \mathrm{H} & 14.28093900 & 9.96697300 & 4.43210900 \\ \mathrm{H} & 15.16277000 & 9.11295600 & 6.60869400 \\ \mathrm{H} & 6.05721100 & 7.51177300 & 6.70339500 \\ \mathrm{H} & 7.12391400 & 6.09737600 & 6.69660800 \\ \mathrm{H} & 6.49495200 & 6.70667100 & 8.97662900 \\ \mathrm{H} & 8.20483800 & 6.84676800 & 8.76329600 \\ \mathrm{H} & 7.64247800 & 9.45168100 & 8.27839000 \\ \mathrm{H} & 6.12186700 & 9.06243900 & 9.03477300\end{array}$

\begin{tabular}{|c|c|c|c|}
\hline \multicolumn{4}{|c|}{$\left({ }^{4-B B N} N^{t B u}\right) Z n(S P h)_{2}$ (No B-S interaction) } \\
\hline $\mathrm{Zn}$ & 5.51125400 & 8.48580500 & 0.53290000 \\
\hline S & 7.35567100 & 7.40268900 & -0.29297500 \\
\hline$S$ & 4.36156700 & 9.80349200 & -0.94505100 \\
\hline $\mathrm{N}$ & 5.66615600 & 9.40870900 & 2.43662500 \\
\hline $\mathrm{N}$ & 4.41845800 & 7.16764700 & 1.72325900 \\
\hline $\mathrm{N}$ & 3.87433000 & 5.94528000 & 1.60191200 \\
\hline C & 7.00027800 & 11.23956300 & 1.55140800 \\
\hline C & 6.28410300 & 10.57766400 & 2.69396700 \\
\hline C & 6.25299800 & 11.13130200 & 3.97948300 \\
\hline C & 5.57403400 & 10.46732300 & 4.99750800 \\
\hline C & 4.93992700 & 9.25877000 & 4.72222600 \\
\hline C & 5.00743200 & 8.75475700 & 3.42305000 \\
\hline C & 4.39509000 & 7.48430500 & 3.02261800 \\
\hline C & 3.80602500 & 6.44008900 & 3.75270700 \\
\hline C & 3.48502000 & 5.45384100 & 2.82267100 \\
\hline C & 2.88354100 & 4.07806600 & 3.08730900 \\
\hline C & 2.54708200 & 3.96939700 & 4.59103400 \\
\hline C & 3.90995800 & 2.96673200 & 2.75329300 \\
\hline C & 1.56941800 & 3.86431100 & 2.29812400 \\
\hline C & 3.77935600 & 5.39452900 & 0.24068000 \\
\hline C & 2.69538900 & 6.07863900 & -0.60285500 \\
\hline C & 2.66966300 & 5.56537500 & -2.05307800 \\
\hline C & 2.24976400 & 4.09568800 & -2.22911700 \\
\hline C & 2.73353300 & 4.19563300 & -5.00274400 \\
\hline C & 3.55081800 & 3.20162600 & -5.89020000 \\
\hline C & 3.62488000 & 1.78429200 & -5.29355500 \\
\hline C & 2.24751000 & 1.20417800 & -4.92214600 \\
\hline C & 1.40658900 & 2.16531600 & -4.02135300 \\
\hline C & 0.12123100 & 2.68122200 & -4.75397100 \\
\hline
\end{tabular}




\begin{tabular}{|c|c|c|c|}
\hline C & 0.44090400 & 3.51790200 & -6.00406300 \\
\hline C & 1.41753900 & 4.66980000 & -5.71375000 \\
\hline C & 7.59711900 & 6.02732200 & 0.82642700 \\
\hline C & 7.82617300 & 6.20059700 & 2.20369100 \\
\hline C & 8.04126200 & 5.10398400 & 3.03996700 \\
\hline C & 8.04827000 & 3.80624000 & 2.52157300 \\
\hline C & 7.83952900 & 3.62098100 & 1.15264400 \\
\hline C & 7.61459600 & 4.71580300 & 0.31631000 \\
\hline C & 3.35846100 & 10.88536500 & 0.06953200 \\
\hline C & 3.41888700 & 12.27494600 & -0.14126600 \\
\hline C & 2.62048900 & 13.14939100 & 0.59780500 \\
\hline C & 1.74515400 & 12.66038600 & 1.56984400 \\
\hline C & 1.67193300 & 11.28228500 & 1.78584900 \\
\hline C & 2.46226800 & 10.40471100 & 1.04200000 \\
\hline B & 2.18961800 & 3.50061100 & -3.69230400 \\
\hline $\mathrm{H}$ & 4.84032200 & 3.11400100 & 3.31137700 \\
\hline $\mathrm{H}$ & 3.49388400 & 1.98949700 & 3.02473000 \\
\hline $\mathrm{H}$ & 4.16993400 & 2.93212500 & 1.69184900 \\
\hline $\mathrm{H}$ & 1.71717500 & 3.85599400 & 1.21523800 \\
\hline $\mathrm{H}$ & 1.13031400 & 2.89936600 & 2.57599900 \\
\hline $\mathrm{H}$ & 0.83998400 & 4.64734200 & 2.53336400 \\
\hline $\mathrm{H}$ & 3.44241600 & 4.06993900 & 5.21380100 \\
\hline $\mathrm{H}$ & 1.82182800 & 4.73073200 & 4.89880800 \\
\hline $\mathrm{H}$ & 2.10898000 & 2.98696400 & 4.79561900 \\
\hline $\mathrm{H}$ & 3.12293700 & 3.14831100 & -6.90124400 \\
\hline $\mathrm{H}$ & 4.57096200 & 3.58339700 & -6.01872500 \\
\hline $\mathrm{H}$ & 4.14512900 & 1.10911100 & -5.98589200 \\
\hline $\mathrm{H}$ & 4.24511600 & 1.82152400 & -4.38303300 \\
\hline $\mathrm{H}$ & 1.69722800 & 0.95915900 & -5.84150800 \\
\hline $\mathrm{H}$ & 2.39502600 & 0.24981500 & -4.40131300 \\
\hline $\mathrm{H}$ & -0.47366900 & 3.29092500 & -4.05668700 \\
\hline $\mathrm{H}$ & -0.51330100 & 1.82592600 & -5.02697400 \\
\hline $\mathrm{H}$ & -0.48886200 & 3.92029200 & -6.42688800 \\
\hline $\mathrm{H}$ & 0.86602200 & 2.86681100 & -6.77836700 \\
\hline $\mathrm{H}$ & 0.91359300 & 5.41912200 & -5.08459200 \\
\hline $\mathrm{H}$ & 1.67714800 & 5.18577400 & -6.64876500 \\
\hline $\mathrm{H}$ & 3.33394600 & 5.09286300 & -4.81327500 \\
\hline $\mathrm{H}$ & 1.07197000 & 1.62820700 & -3.12352500 \\
\hline $\mathrm{H}$ & 2.37603400 & 9.33380200 & 1.20324500 \\
\hline $\mathrm{H}$ & 0.98647600 & 10.88384900 & 2.53085500 \\
\hline $\mathrm{H}$ & 1.12446200 & 13.34185800 & 2.14564200 \\
\hline $\mathrm{H}$ & 2.68513900 & 14.21916600 & 0.41209200 \\
\hline $\mathrm{H}$ & 4.09580400 & 12.66239000 & -0.89752800 \\
\hline $\mathrm{H}$ & 7.85027700 & 7.20619800 & 2.61385600 \\
\hline
\end{tabular}




$\begin{array}{cccc}\mathrm{H} & 8.21935100 & 5.26693400 & 4.10071800 \\ \mathrm{H} & 8.22949100 & 2.95429700 & 3.17181300 \\ \mathrm{H} & 7.85527600 & 2.61861500 & 0.73036300 \\ \mathrm{H} & 7.45872100 & 4.56522000 & -0.74835700 \\ \mathrm{H} & 5.53871800 & 10.88773900 & 5.99851900 \\ \mathrm{H} & 4.39958000 & 8.71785000 & 5.49135300 \\ \mathrm{H} & 6.75874600 & 12.07222100 & 4.16773000 \\ \mathrm{H} & 7.73675900 & 10.55549500 & 1.11364600 \\ \mathrm{H} & 6.28982200 & 11.49815200 & 0.75798200 \\ \mathrm{H} & 7.51226300 & 12.14808700 & 1.87905700 \\ \mathrm{H} & 3.65667100 & 6.38729900 & 4.81908200 \\ \mathrm{H} & 4.76544500 & 5.53204100 & -0.21441200 \\ \mathrm{H} & 3.60374900 & 4.32519900 & 0.33241600 \\ \mathrm{H} & 1.71521700 & 5.92724000 & -0.12955700 \\ \mathrm{H} & 2.88992900 & 7.15734300 & -0.61198400 \\ \mathrm{H} & 1.98162200 & 6.20768400 & -2.61725800 \\ \mathrm{H} & 3.65816900 & 5.72825000 & -2.50293800 \\ \mathrm{H} & 2.96083100 & 3.42695300 & -1.70604200 \\ \mathrm{H} & 1.29064600 & 3.90745100 & -1.71677200\end{array}$

\begin{tabular}{|c|c|c|c|}
\hline \multicolumn{4}{|c|}{$\left(\left(^{4-B B N} N^{t B u}\right) Z n(S P h)_{2}(B-S=2.128 \AA)\right.$} \\
\hline $\mathrm{Zn}$ & 10.34835400 & 7.95948500 & 8.45510500 \\
\hline $\mathrm{S}$ & 9.99694500 & 9.33802500 & 10.29723600 \\
\hline$S$ & 10.97331100 & 5.75139600 & 8.45903000 \\
\hline $\mathrm{N}$ & 11.95158600 & 8.62175000 & 7.25492600 \\
\hline $\mathrm{N}$ & 9.29899700 & 8.45229500 & 6.61307800 \\
\hline $\mathrm{N}$ & 8.10061100 & 8.22063900 & 6.02119400 \\
\hline C & 13.53610900 & 8.67753500 & 9.10922800 \\
\hline C & 13.23078800 & 8.79895300 & 7.64287500 \\
\hline C & 14.22995700 & 9.06022100 & 6.69870900 \\
\hline C & 13.89984300 & 9.12767600 & 5.34779800 \\
\hline C & 12.57875400 & 8.93202900 & 4.95796500 \\
\hline C & 11.62413000 & 8.67729100 & 5.94464600 \\
\hline C & 10.21045800 & 8.45405900 & 5.62751100 \\
\hline C & 9.59366100 & 8.21028900 & 4.39247300 \\
\hline C & 8.23936400 & 8.06219300 & 4.66164100 \\
\hline C & 7.12686700 & 7.75984700 & 3.65852500 \\
\hline C & 6.27984200 & 6.54325500 & 4.10367000 \\
\hline C & 7.77953500 & 7.39466200 & 2.30520800 \\
\hline C & 6.22871800 & 8.99942100 & 3.41366500 \\
\hline C & 6.85654500 & 8.45521200 & 6.78266600 \\
\hline C & 6.48603700 & 7.41719300 & 7.85152800 \\
\hline C & 7.06788200 & 8.86922500 & 9.93727500 \\
\hline
\end{tabular}




\begin{tabular}{|c|c|c|c|}
\hline C & 7.79815600 & 10.44200600 & 12.09316000 \\
\hline C & 6.39792000 & 10.24769100 & 12.74324800 \\
\hline C & 6.21581700 & 8.92942600 & 13.53373900 \\
\hline$C$ & 6.83426700 & 7.67618500 & 12.86985800 \\
\hline C & 8.24460500 & 7.87971400 & 12.24296100 \\
\hline C & 9.34390600 & 8.12789600 & 13.30440000 \\
\hline C & 9.25854800 & 9.48247500 & 14.04410200 \\
\hline C & 8.90642500 & 10.69046600 & 13.14680100 \\
\hline C & 10.21202600 & 11.00925600 & 9.66567800 \\
\hline C & 11.16589900 & 11.83323700 & 10.28408100 \\
\hline C & 11.37451400 & 13.13917000 & 9.83723400 \\
\hline C & 10.63461200 & 13.64322100 & 8.76622700 \\
\hline C & 9.68392000 & 12.83043400 & 8.14584000 \\
\hline C & 9.47217800 & 11.52390700 & 8.59046400 \\
\hline C & 9.62100700 & 4.73144300 & 9.04296200 \\
\hline C & 8.75289400 & 4.11563600 & 8.12594000 \\
\hline C & 7.72856500 & 3.27527400 & 8.56702300 \\
\hline C & 7.55353600 & 3.03269900 & 9.93141200 \\
\hline C & 8.41877800 & 3.63217700 & 10.84954700 \\
\hline C & 9.44606200 & 4.46997900 & 10.41109400 \\
\hline B & 8.08134200 & 9.11555000 & 11.19687700 \\
\hline $\mathrm{H}$ & 8.73350400 & 10.89980900 & 8.10094200 \\
\hline $\mathrm{H}$ & 9.09982700 & 13.21392700 & 7.31285300 \\
\hline $\mathrm{H}$ & 10.79561000 & 14.66038400 & 8.41936300 \\
\hline $\mathrm{H}$ & 12.11552000 & 13.76296500 & 10.33059300 \\
\hline $\mathrm{H}$ & 11.73791800 & 11.44570100 & 11.12179700 \\
\hline $\mathrm{H}$ & 10.12016600 & 4.92709500 & 11.12857700 \\
\hline $\mathrm{H}$ & 8.29645800 & 3.44822500 & 11.91400300 \\
\hline $\mathrm{H}$ & 6.75597400 & 2.37926900 & 10.27504000 \\
\hline $\mathrm{H}$ & 7.06885400 & 2.80493800 & 7.84115700 \\
\hline $\mathrm{H}$ & 8.89652600 & 4.29343600 & 7.06382700 \\
\hline $\mathrm{H}$ & 8.49506000 & 6.93013900 & 11.74476800 \\
\hline $\mathrm{H}$ & 7.73033800 & 11.35479100 & 11.47896700 \\
\hline $\mathrm{H}$ & 9.81473900 & 11.00918100 & 12.61927500 \\
\hline $\mathrm{H}$ & 8.63007200 & 11.53825900 & 13.79477400 \\
\hline $\mathrm{H}$ & 8.52627500 & 9.40807800 & 14.85484000 \\
\hline $\mathrm{H}$ & 10.21873400 & 9.67760500 & 14.54386000 \\
\hline $\mathrm{H}$ & 9.33939100 & 7.31934500 & 14.05343000 \\
\hline $\mathrm{H}$ & 10.31926200 & 8.06616600 & 12.80395900 \\
\hline $\mathrm{H}$ & 6.63969500 & 9.04918700 & 14.53566700 \\
\hline $\mathrm{H}$ & 5.14206200 & 8.75466100 & 13.69530900 \\
\hline $\mathrm{H}$ & 6.86699300 & 6.86606100 & 13.61592000 \\
\hline $\mathrm{H}$ & 6.15476600 & 7.32350200 & 12.08131500 \\
\hline $\mathrm{H}$ & 6.17329300 & 11.09186700 & 13.41485800 \\
\hline
\end{tabular}




$\begin{array}{cccc}\mathrm{H} & 5.63954700 & 10.29294700 & 11.94988300 \\ \mathrm{H} & 14.59294300 & 8.87486200 & 9.30652500 \\ \mathrm{H} & 12.93027600 & 9.37784100 & 9.69320700 \\ \mathrm{H} & 13.29569500 & 7.66822100 & 9.46450800 \\ \mathrm{H} & 6.91373000 & 5.66187200 & 4.24911000 \\ \mathrm{H} & 5.72977200 & 6.71529600 & 5.03188000 \\ \mathrm{H} & 5.54514900 & 6.30723200 & 3.32560300 \\ \mathrm{H} & 6.82903100 & 9.85440000 & 3.08402600 \\ \mathrm{H} & 5.50120700 & 8.77228200 & 2.62611400 \\ \mathrm{H} & 5.66457700 & 9.30898400 & 4.29788600 \\ \mathrm{H} & 8.44343100 & 6.52876000 & 2.39868800 \\ \mathrm{H} & 6.99742700 & 7.14270400 & 1.58151300 \\ \mathrm{H} & 8.35391700 & 8.23078700 & 1.89207000 \\ \mathrm{H} & 10.07166900 & 8.11756200 & 3.43103500 \\ \mathrm{H} & 12.28751000 & 8.98185200 & 3.91492800 \\ \mathrm{H} & 14.66572700 & 9.32950500 & 4.60446700 \\ \mathrm{H} & 15.25307700 & 9.20066800 & 7.03036400 \\ \mathrm{H} & 6.94648800 & 9.44841300 & 7.23045500 \\ \mathrm{H} & 6.06603800 & 8.51372500 & 6.04024300 \\ \mathrm{H} & 5.41120900 & 7.55312200 & 8.03857300 \\ \mathrm{H} & 6.60935600 & 6.40849400 & 7.43706400 \\ \mathrm{H} & 7.15105900 & 9.72213100 & 9.24572400 \\ \mathrm{H} & 6.02791800 & 8.91088900 & 10.29606200 \\ \mathrm{C} & 7.23891700 & 7.54171900 & 9.18477300 \\ \mathrm{H} & 8.29978000 & 7.33904300 & 8.99001300 \\ \mathrm{H} & 6.92488400 & 6.70977900 & 9.82526800 \\ & & & \end{array}$

$\begin{array}{lccc}\left.\mathbf{(}^{4-B B N} \mathbf{N N}^{\mathrm{tBu}}\right) \mathbf{Z n}(\mathbf{S P h})_{\mathbf{2}}(\mathrm{B}-\mathbf{S}=\mathbf{2 . 1 8 3} \AA \mathbf{\AA}) & \\ \mathrm{Zn} & 10.35067500 & 7.96712200 & 8.45334500 \\ \mathrm{~S} & 10.00227500 & 9.33529000 & 10.29972900 \\ \mathrm{~S} & 10.96821500 & 5.75646800 & 8.45331100 \\ \mathrm{~N} & 11.95283500 & 8.62385200 & 7.24940800 \\ \mathrm{~N} & 9.29973800 & 8.45610900 & 6.60980400 \\ \mathrm{~N} & 8.10079400 & 8.22323900 & 6.01970800 \\ \mathrm{C} & 13.53786500 & 8.68976100 & 9.10296900 \\ \mathrm{C} & 13.23200100 & 8.80393700 & 7.63617100 \\ \mathrm{C} & 14.23051400 & 9.06229600 & 6.69048000 \\ \mathrm{C} & 13.89979400 & 9.12403400 & 5.33944800 \\ \mathrm{C} & 12.57866800 & 8.92590800 & 4.95095800 \\ \mathrm{C} & 11.62473500 & 8.67438600 & 5.93911300 \\ \mathrm{C} & 10.21079400 & 8.45030100 & 5.62383500 \\ \mathrm{C} & 9.59307700 & 8.20009400 & 4.39054300 \\ \mathrm{C} & 8.23858700 & 8.05615400 & 4.66114800 \\ \mathrm{C} & 7.12484100 & 7.75105400 & 3.66030300\end{array}$




\begin{tabular}{|c|c|c|c|}
\hline C & 6.27069400 & 6.54289200 & 4.11466800 \\
\hline C & 7.77605600 & 7.37137600 & 2.31031500 \\
\hline C & 6.23356400 & 8.99355400 & 3.40540500 \\
\hline C & 6.85796000 & 8.46313000 & 6.78125300 \\
\hline C & 6.47999400 & 7.42249000 & 7.84529200 \\
\hline C & 7.04884100 & 8.86837400 & 9.93712400 \\
\hline C & 7.76030000 & 10.43196400 & 12.10601200 \\
\hline C & 6.35756400 & 10.23890100 & 12.75344000 \\
\hline C & 6.17312500 & 8.92057800 & 13.54325000 \\
\hline C & 6.78869000 & 7.66698500 & 12.87741400 \\
\hline C & 8.20171400 & 7.86820600 & 12.25326100 \\
\hline C & 9.30057100 & 8.11339900 & 13.31554800 \\
\hline C & 9.21680900 & 9.46696100 & 14.05718800 \\
\hline C & 8.86770900 & 10.67670400 & 13.16110400 \\
\hline C & 10.21553300 & 11.01035200 & 9.68085600 \\
\hline C & 11.14734200 & 11.84190300 & 10.32289200 \\
\hline C & 11.34994600 & 13.15269700 & 9.88786700 \\
\hline C & 10.62719600 & 13.65472700 & 8.80421500 \\
\hline C & 9.69941000 & 12.83450800 & 8.15947800 \\
\hline C & 9.49276700 & 11.52341700 & 8.59297200 \\
\hline C & 9.61712500 & 4.73930900 & 9.04445600 \\
\hline C & 8.74515400 & 4.12214300 & 8.13196900 \\
\hline C & 7.72163100 & 3.28372800 & 8.57854500 \\
\hline C & 7.55121800 & 3.04428800 & 9.94407600 \\
\hline C & 8.42032300 & 3.64497400 & 10.85776700 \\
\hline C & 9.44674700 & 4.48092400 & 10.41377500 \\
\hline B & 8.03421900 & 9.10457900 & 11.21570800 \\
\hline $\mathrm{H}$ & 8.77161100 & 10.89404400 & 8.08408400 \\
\hline $\mathrm{H}$ & 9.12876900 & 13.21580900 & 7.31618100 \\
\hline $\mathrm{H}$ & 10.78397900 & 14.67554700 & 8.46629300 \\
\hline $\mathrm{H}$ & 12.07369300 & 13.78175100 & 10.39986500 \\
\hline $\mathrm{H}$ & 11.70761900 & 11.45562600 & 11.16905600 \\
\hline $\mathrm{H}$ & 10.12400700 & 4.93883500 & 11.12780300 \\
\hline $\mathrm{H}$ & 8.30177900 & 3.46332000 & 11.92305300 \\
\hline $\mathrm{H}$ & 6.75436800 & 2.39225200 & 10.29197800 \\
\hline $\mathrm{H}$ & 7.05893100 & 2.81236400 & 7.85607100 \\
\hline $\mathrm{H}$ & 8.88523200 & 4.29746900 & 7.06896900 \\
\hline $\mathrm{H}$ & 8.45028900 & 6.91988000 & 11.75243900 \\
\hline $\mathrm{H}$ & 7.69519400 & 11.34401200 & 11.49083200 \\
\hline $\mathrm{H}$ & 9.77665300 & 10.99342400 & 12.63390600 \\
\hline $\mathrm{H}$ & 8.59259400 & 11.52449100 & 13.80953600 \\
\hline $\mathrm{H}$ & 8.48407000 & 9.39269200 & 14.86754600 \\
\hline $\mathrm{H}$ & 10.17709400 & 9.65979900 & 14.55752200 \\
\hline $\mathrm{H}$ & 9.29487300 & 7.30360600 & 14.06318300 \\
\hline
\end{tabular}




$\begin{array}{cccc}\mathrm{H} & 10.27550400 & 8.05136000 & 12.81443000 \\ \mathrm{H} & 6.59776900 & 9.03856600 & 14.54499800 \\ \mathrm{H} & 5.09909400 & 8.74784100 & 13.70487100 \\ \mathrm{H} & 6.81796700 & 6.85451700 & 13.62092400 \\ \mathrm{H} & 6.10982500 & 7.31893000 & 12.08610900 \\ \mathrm{H} & 6.13259600 & 11.08352200 & 13.42421700 \\ \mathrm{H} & 5.60107200 & 10.28443100 & 11.95820700 \\ \mathrm{H} & 14.59589100 & 8.88279600 & 9.29813300 \\ \mathrm{H} & 12.93603900 & 9.39683000 & 9.68299300 \\ \mathrm{H} & 13.29233000 & 7.68402700 & 9.46473000 \\ \mathrm{H} & 6.89965400 & 5.65952200 & 4.26920100 \\ \mathrm{H} & 5.71944800 & 6.72609300 & 5.04002800 \\ \mathrm{H} & 5.53645200 & 6.30382500 & 3.33711300 \\ \mathrm{H} & 6.83841400 & 9.84207500 & 3.06746100 \\ \mathrm{H} & 5.50367100 & 8.76367800 & 2.62084800 \\ \mathrm{H} & 5.67253200 & 9.31414700 & 4.28769100 \\ \mathrm{H} & 8.43540400 & 6.50283900 & 2.41128100 \\ \mathrm{H} & 6.99293300 & 7.11759400 & 1.58833800 \\ \mathrm{H} & 8.35498200 & 8.20104800 & 1.89058400 \\ \mathrm{H} & 10.07057300 & 8.10064100 & 3.42950900 \\ \mathrm{H} & 12.28681400 & 8.97165100 & 3.90789900 \\ \mathrm{H} & 14.66520700 & 9.32355200 & 4.59500400 \\ \mathrm{H} & 15.25363600 & 9.20507100 & 7.02113000 \\ \mathrm{H} & 6.95493900 & 9.45359700 & 7.23351100 \\ \mathrm{H} & 6.06837000 & 8.53115700 & 6.03854100 \\ \mathrm{H} & 5.40428300 & 7.55866300 & 8.02706600 \\ \mathrm{H} & 6.60471300 & 6.41482400 & 7.42888100 \\ \mathrm{H} & 7.15096900 & 9.72403300 & 9.25184100 \\ \mathrm{H} & 6.00261300 & 8.91541100 & 10.27798000 \\ \mathrm{C} & 7.22592400 & 7.54269700 & 9.18282900 \\ \mathrm{H} & 8.28822700 & 7.34259600 & 8.99455000 \\ \mathrm{H} & 6.90798700 & 6.70896500 & 9.81913000\end{array}$

$\left.{ }^{(3-B P i n} N^{\text {tBu}}\right) Z n(S P h)_{2}$ (No B-S interaction)

Zn $\quad 9.84406800 \quad 11.32667400 \quad 26.84395700$

S $\quad \begin{array}{llll}10.14458700 & 10.85116800 & 29.05786000\end{array}$

S $\quad 8.33244300 \quad 12.86412900 \quad 26.07180000$

$\begin{array}{lllll}N & 9.89131400 & 9.51977200 & 25.70978500\end{array}$

N $\quad \begin{array}{llll}N & 11.61228000 & 11.55065400 & 25.75440600\end{array}$

$\begin{array}{lllll}\mathrm{N} & 12.50640500 & 12.52778900 & 25.53075400\end{array}$

C $\quad \begin{array}{llll}7.87555300 & 8.66820200 & 26.77542800\end{array}$

$\begin{array}{llll}\text { C } & 9.00461900 & 8.50857200 & 25.79681100\end{array}$

$\begin{array}{llll}\text { C } & 9.13933000 & 7.37116000 & 24.99156200\end{array}$ 


\begin{tabular}{|c|c|c|c|}
\hline C & 10.19846400 & 7.28844700 & 24.09085000 \\
\hline C & 11.10697300 & 8.33932900 & 24.00512400 \\
\hline$C$ & 10.92181200 & 9.44723600 & 24.83381200 \\
\hline$C$ & 11.81379500 & 10.61002700 & 24.82599400 \\
\hline$C$ & 12.87503200 & 10.99068200 & 23.98903500 \\
\hline C & 13.30616500 & 12.23017500 & 24.45480400 \\
\hline$C$ & 14.39224300 & 13.12589000 & 23.86919300 \\
\hline C & 13.77560000 & 14.44349400 & 23.33639500 \\
\hline C & 15.50745400 & 13.42488300 & 24.89931600 \\
\hline C & 15.04167200 & 12.39394600 & 22.67429800 \\
\hline C & 12.53319900 & 13.62852700 & 26.50731300 \\
\hline C & 13.17842500 & 13.21415200 & 27.83590500 \\
\hline C & 13.14748400 & 14.35264400 & 28.87236100 \\
\hline 0 & 13.30354600 & 14.25338700 & 31.47020300 \\
\hline $\mathrm{O}$ & 14.99068400 & 13.22502300 & 30.31293700 \\
\hline C & 14.29320400 & 13.88482400 & 32.47411400 \\
\hline C & 15.17764800 & 12.82860100 & 31.70277200 \\
\hline C & 15.04924300 & 15.17175800 & 32.83258700 \\
\hline C & 13.55913500 & 13.34798300 & 33.70145800 \\
\hline C & 14.65656100 & 11.38971300 & 31.82175800 \\
\hline C & 16.67129500 & 12.87412500 & 32.02104800 \\
\hline C & 9.24139000 & 12.15571700 & 29.89921600 \\
\hline C & 7.86752500 & 12.36448100 & 29.69527300 \\
\hline C & 7.18642700 & 13.36041100 & 30.39659800 \\
\hline C & 7.85781500 & 14.16006300 & 31.32406800 \\
\hline C & 9.22209900 & 13.95540700 & 31.53838500 \\
\hline C & 9.90822100 & 12.96462100 & 30.83366100 \\
\hline C & 8.78858500 & 13.01160300 & 24.34515500 \\
\hline C & 8.51497200 & 11.99701100 & 23.40989800 \\
\hline C & 8.84052700 & 12.15487800 & 22.06154600 \\
\hline C & 9.43570900 & 13.33494700 & 21.60973300 \\
\hline C & 9.69875800 & 14.35736400 & 22.52489000 \\
\hline C & 9.38266900 & 14.19685200 & 23.87538800 \\
\hline B & 13.82095200 & 13.94243100 & 30.23688600 \\
\hline $\mathrm{H}$ & 16.29803400 & 14.01527500 & 24.42198400 \\
\hline $\mathrm{H}$ & 15.14945300 & 13.99360100 & 25.76103600 \\
\hline $\mathrm{H}$ & 15.95643600 & 12.49724300 & 25.27120300 \\
\hline $\mathrm{H}$ & 14.54792500 & 15.03084900 & 22.82630900 \\
\hline $\mathrm{H}$ & 12.96986100 & 14.23704700 & 22.62435100 \\
\hline $\mathrm{H}$ & 13.36047800 & 15.06847100 & 24.13233500 \\
\hline $\mathrm{H}$ & 15.83068100 & 13.02237900 & 22.24808600 \\
\hline $\mathrm{H}$ & 15.49749900 & 11.44603700 & 22.98082900 \\
\hline $\mathrm{H}$ & 14.31326600 & 12.19008000 & 21.88224100 \\
\hline $\mathrm{H}$ & 13.26178300 & 10.45759900 & 23.13536600 \\
\hline
\end{tabular}




$\begin{array}{lccc}\mathrm{H} & 11.94422800 & 8.30575200 & 23.31674900 \\ \mathrm{H} & 10.31547600 & 6.41150900 & 23.46040500 \\ \mathrm{H} & 8.41529300 & 6.56784400 & 25.07656900 \\ \mathrm{H} & 8.26515600 & 8.84361500 & 27.78506400 \\ \mathrm{H} & 7.26748000 & 9.54265100 & 26.51279400 \\ \mathrm{H} & 7.23256500 & 7.78402700 & 26.78557000 \\ \mathrm{H} & 8.02838100 & 11.08633400 & 23.74701200 \\ \mathrm{H} & 8.61569000 & 11.35528600 & 21.35900800 \\ \mathrm{H} & 9.67855100 & 13.46119100 & 20.55779100 \\ \mathrm{H} & 10.14579700 & 15.28958500 & 22.18603200 \\ \mathrm{H} & 9.58051400 & 14.99874100 & 24.58114100 \\ \mathrm{H} & 7.33204500 & 11.74623100 & 28.98085200 \\ \mathrm{H} & 6.12468300 & 13.50917300 & 30.21511000 \\ \mathrm{H} & 7.32470400 & 14.93452100 & 31.86948500 \\ \mathrm{H} & 9.76511800 & 14.57278600 & 32.25010700 \\ \mathrm{H} & 10.97098200 & 12.82416700 & 31.00318000 \\ \mathrm{H} & 12.94980900 & 14.14371700 & 34.14210600 \\ \mathrm{H} & 12.89505500 & 12.51960100 & 33.44536700 \\ \mathrm{H} & 14.27018500 & 13.00557500 & 34.46223500 \\ \mathrm{H} & 14.80585300 & 10.98872200 & 32.82983300 \\ \mathrm{H} & 13.59201500 & 11.32451000 & 31.57562200 \\ \mathrm{H} & 15.20169800 & 10.75592800 & 31.11527500 \\ \mathrm{H} & 17.10203900 & 13.85509600 & 31.80739300 \\ \mathrm{H} & 16.85326100 & 12.63403700 & 33.07495200 \\ \mathrm{H} & 17.19863900 & 12.13397500 & 31.41051100 \\ \mathrm{H} & 15.57775400 & 15.57875100 & 31.96440900 \\ \mathrm{H} & 14.32880900 & 15.92280000 & 33.17109500 \\ \mathrm{H} & 15.77620500 & 15.00511200 & 33.63455900 \\ \mathrm{H} & 13.67249600 & 15.23602600 & 28.47302900 \\ \mathrm{H} & 12.11392200 & 14.66909800 & 29.06005200 \\ \mathrm{H} & 14.21186000 & 12.89360000 & 27.65867900 \\ \mathrm{H} & 12.63459500 & 12.34694400 & 28.22748200 \\ \mathrm{H} & 13.05923800 & 14.46786100 & 26.05361500 \\ & 11.49193800 & 13.93118200 & 26.65876700\end{array}$

$\begin{array}{lccc}\left.\mathbf{(}^{\text {-BPin }} \mathbf{N N}^{\text {tBu}}\right) Z \mathbf{Z n}(\mathbf{S P h})_{\mathbf{2}}(\mathbf{B}-\mathbf{S}=\mathbf{2 . 1 2 8} \AA \mathbf{\AA}) & \\ \mathrm{Zn} & 9.89269900 & 7.20322100 & 8.19132700 \\ \mathrm{~S} & 9.48246500 & 8.61117100 & 10.00553400 \\ \mathrm{~S} & 9.78885300 & 4.93535200 & 8.24522000 \\ \mathrm{~N} & 11.57947300 & 7.90175900 & 7.13109100 \\ \mathrm{~N} & 8.99144100 & 7.91633800 & 6.43006900 \\ \mathrm{~N} & 7.76087600 & 8.11679400 & 5.92090800 \\ \mathrm{C} & 13.09701800 & 7.20436300 & 8.90009900\end{array}$




\begin{tabular}{|c|c|c|c|}
\hline C & 12.85814200 & 7.79989800 & 7.54138600 \\
\hline C & 13.90428300 & 8.23933200 & 6.72187900 \\
\hline C & 13.61597000 & 8.78297000 & 5.47282600 \\
\hline C & 12.29079400 & 8.87981100 & 5.05743600 \\
\hline C & 11.29029400 & 8.42685400 & 5.91824400 \\
\hline C & 9.86381600 & 8.47518600 & 5.58032000 \\
\hline C & 9.17628100 & 9.03773200 & 4.49505100 \\
\hline C & 7.82509400 & 8.79630300 & 4.72968200 \\
\hline C & 6.64185400 & 9.20588200 & 3.85736600 \\
\hline C & 5.81419100 & 7.97556000 & 3.41131200 \\
\hline C & 7.18566100 & 9.88545500 & 2.58082400 \\
\hline C & 5.74725500 & 10.23725200 & 4.58874200 \\
\hline C & 6.59982500 & 7.50703100 & 6.60772700 \\
\hline C & 6.68873800 & 7.57555600 & 8.13323100 \\
\hline C & 6.78204800 & 8.98430700 & 8.74042900 \\
\hline C & 10.30193200 & 10.15886600 & 9.60228400 \\
\hline C & 11.26776100 & 10.64256100 & 10.49904900 \\
\hline C & 11.92391700 & 11.85031500 & 10.25615300 \\
\hline C & 11.62420700 & 12.59759700 & 9.11564000 \\
\hline C & 10.66192900 & 12.12533400 & 8.22107800 \\
\hline C & 10.00528900 & 10.91657200 & 8.45963400 \\
\hline C & 10.13911400 & 4.46951800 & 9.94385200 \\
\hline C & 11.15986900 & 3.53638700 & 10.19129000 \\
\hline C & 11.43123400 & 3.10343900 & 11.49048900 \\
\hline C & 10.69271100 & 3.59825100 & 12.56688800 \\
\hline C & 9.67496800 & 4.52460000 & 12.32755100 \\
\hline C & 9.39096200 & 4.95452500 & 11.03003300 \\
\hline B & 7.39788100 & 8.96993200 & 10.23830200 \\
\hline $\mathrm{H}$ & 9.25573300 & 10.56621900 & 7.75878200 \\
\hline $\mathrm{H}$ & 10.41468100 & 12.70207600 & 7.33297900 \\
\hline $\mathrm{H}$ & 12.13164800 & 13.54010700 & 8.92802900 \\
\hline $\mathrm{H}$ & 12.66740900 & 12.20856400 & 10.96386100 \\
\hline $\mathrm{H}$ & 11.49459500 & 10.06854700 & 11.39251700 \\
\hline $\mathrm{H}$ & 8.58572300 & 5.66526300 & 10.86737000 \\
\hline $\mathrm{H}$ & 9.08685800 & 4.91447500 & 13.15483500 \\
\hline $\mathrm{H}$ & 10.90490800 & 3.26387800 & 13.57915900 \\
\hline $\mathrm{H}$ & 12.22451700 & 2.37858800 & 11.65932000 \\
\hline $\mathrm{H}$ & 11.73530300 & 3.14881000 & 9.35515800 \\
\hline $\mathrm{H}$ & 14.16533300 & 7.14868200 & 9.12484100 \\
\hline $\mathrm{H}$ & 12.60941300 & 7.80892100 & 9.67374400 \\
\hline $\mathrm{H}$ & 12.67101800 & 6.19593300 & 8.96011800 \\
\hline $\mathrm{H}$ & 6.44738300 & 7.24642600 & 2.89384400 \\
\hline $\mathrm{H}$ & 5.32386300 & 7.46474000 & 4.24337000 \\
\hline $\mathrm{H}$ & 5.03069000 & 8.29603300 & 2.71530100 \\
\hline
\end{tabular}




$\begin{array}{cccc}\mathrm{H} & 6.32682400 & 11.12391500 & 4.86741300 \\ \mathrm{H} & 4.93630800 & 10.55664600 & 3.92405100 \\ \mathrm{H} & 5.28988700 & 9.84235800 & 5.49969900 \\ \mathrm{H} & 7.82508500 & 9.20872700 & 2.00357500 \\ \mathrm{H} & 6.34748000 & 10.18056000 & 1.94119900 \\ \mathrm{H} & 7.75800300 & 10.78952300 & 2.81534200 \\ \mathrm{H} & 9.59669800 & 9.56493900 & 3.65392000 \\ \mathrm{H} & 12.03428600 & 9.29132200 & 4.08753300 \\ \mathrm{H} & 14.41787600 & 9.12666300 & 4.82572700 \\ \mathrm{H} & 14.92807000 & 8.15190600 & 7.06939800 \\ \mathrm{H} & 5.71745700 & 8.03901700 & 6.25676400 \\ \mathrm{H} & 6.52988600 & 6.46544100 & 6.27197900 \\ \mathrm{H} & 5.79890400 & 7.06359800 & 8.51748400 \\ \mathrm{H} & 7.51744600 & 6.93707200 & 8.45569300 \\ \mathrm{H} & 7.35233800 & 9.65312300 & 8.08339000 \\ \mathrm{H} & 5.77239800 & 9.41954100 & 8.79717200 \\ \mathrm{O} & 7.25322400 & 10.16376800 & 10.99209600 \\ \mathrm{O} & 7.02330100 & 7.85331200 & 11.06067200 \\ \mathrm{C} & 7.05174500 & 9.82432300 & 12.37393700 \\ \mathrm{C} & 6.47162600 & 8.35447500 & 12.28982200 \\ \mathrm{C} & 4.93897700 & 8.32208400 & 12.14418700 \\ \mathrm{C} & 6.88900400 & 7.42777200 & 13.43537600 \\ \mathrm{C} & 6.09382900 & 10.86034800 & 12.97317800 \\ \mathrm{C} & 8.39674100 & 9.90669000 & 13.11896600 \\ \mathrm{H} & 4.63156800 & 7.30061400 & 11.89615800 \\ \mathrm{H} & 4.42808200 & 8.62107800 & 13.06679600 \\ \mathrm{H} & 4.60660400 & 8.98063300 & 11.33542300 \\ \mathrm{H} & 7.97553200 & 7.32813700 & 13.49380200 \\ \mathrm{H} & 6.51860900 & 7.79542900 & 14.40023100 \\ \mathrm{H} & 6.46686400 & 6.43006000 & 13.27097600 \\ \mathrm{H} & 6.57561200 & 11.84423100 & 12.97302000 \\ \mathrm{H} & 5.17345300 & 10.94003900 & 12.38984000 \\ \mathrm{H} & 5.83342700 & 10.61145200 & 14.00915000 \\ \mathrm{H} & 8.82756600 & 10.89987300 & 12.95339600 \\ \mathrm{H} & 8.26867500 & 9.76325400 & 14.19840100 \\ & 9.10940700 & 9.16373400 & 12.75244400\end{array}$

$\left(^{3-B P i n} N^{\text {BBu }}\right) Z n(S P h)_{2}(B-S=2.183 \AA$ A

$\begin{array}{cccc}\text { Zn } & 9.89161100 & 7.20820400 & 8.19848300 \\ \mathrm{~S} & 9.46833900 & 8.61452100 & 10.00547000 \\ \mathrm{~S} & 9.78208100 & 4.93932100 & 8.24085100 \\ \mathrm{~N} & 11.57825600 & 7.90721800 & 7.13997200 \\ \mathrm{~N} & 8.99219600 & 7.91651000 & 6.43096900\end{array}$




\begin{tabular}{|c|c|c|c|}
\hline $\mathrm{N}$ & 7.76283400 & 8.11407500 & 5.91769300 \\
\hline C & 13.09187800 & 7.20714700 & 8.91089700 \\
\hline C & 12.85591400 & 7.80719200 & 7.55367400 \\
\hline C & 13.90333100 & 8.25226100 & 6.73885400 \\
\hline C & 13.61719200 & 8.79971900 & 5.49097300 \\
\hline C & 12.29298000 & 8.89452800 & 5.07201500 \\
\hline C & 11.29116400 & 8.43580400 & 5.92823500 \\
\hline C & 9.86554300 & 8.48091100 & 5.58606400 \\
\hline C & 9.18000500 & 9.04446800 & 4.49989100 \\
\hline C & 7.82891600 & 8.79744700 & 4.72858400 \\
\hline C & 6.64750900 & 9.20528800 & 3.85294900 \\
\hline C & 5.82947400 & 7.97271700 & 3.39547300 \\
\hline C & 7.19347200 & 9.89573900 & 2.58315900 \\
\hline C & 5.74306800 & 10.22703700 & 4.58573500 \\
\hline C & 6.60242300 & 7.49430900 & 6.59614600 \\
\hline C & 6.67812400 & 7.56508800 & 8.12233300 \\
\hline C & 6.75038500 & 8.97565200 & 8.72698000 \\
\hline C & 10.29298100 & 10.15943800 & 9.60564100 \\
\hline C & 11.26019200 & 10.64210700 & 10.50201400 \\
\hline C & 11.91634900 & 11.85012700 & 10.26064000 \\
\hline C & 11.61623900 & 12.59958300 & 9.12157300 \\
\hline C & 10.65289300 & 12.12898000 & 8.22725600 \\
\hline C & 9.99576600 & 10.92029300 & 8.46480300 \\
\hline C & 10.11501100 & 4.46173000 & 9.93932100 \\
\hline C & 11.12665000 & 3.51961900 & 10.19018500 \\
\hline C & 11.38342000 & 3.07693700 & 11.48903800 \\
\hline C & 10.63932700 & 3.57084200 & 12.56202500 \\
\hline C & 9.63072100 & 4.50631300 & 12.31947900 \\
\hline C & 9.36134300 & 4.94584000 & 11.02211100 \\
\hline B & 7.32765400 & 8.97522500 & 10.23536000 \\
\hline $\mathrm{H}$ & 9.24459300 & 10.57168000 & 7.76460000 \\
\hline $\mathrm{H}$ & 10.40489500 & 12.70729100 & 7.34033700 \\
\hline $\mathrm{H}$ & 12.12401500 & 13.54213400 & 8.93504400 \\
\hline $\mathrm{H}$ & 12.66078900 & 12.20677000 & 10.96823900 \\
\hline $\mathrm{H}$ & 11.48848200 & 10.06612300 & 11.39390200 \\
\hline $\mathrm{H}$ & 8.56263600 & 5.66310300 & 10.85614000 \\
\hline $\mathrm{H}$ & 9.03861700 & 4.89587400 & 13.14404900 \\
\hline $\mathrm{H}$ & 10.84022200 & 3.22886600 & 13.57406200 \\
\hline $\mathrm{H}$ & 12.16978800 & 2.34516700 & 11.66037300 \\
\hline $\mathrm{H}$ & 11.70658600 & 3.13291600 & 9.35677800 \\
\hline $\mathrm{H}$ & 14.15952700 & 7.15424200 & 9.13947500 \\
\hline $\mathrm{H}$ & 12.59881400 & 7.80683500 & 9.68486600 \\
\hline $\mathrm{H}$ & 12.66908100 & 6.19701800 & 8.96505300 \\
\hline $\mathrm{H}$ & 6.46952900 & 7.25070000 & 2.87648300 \\
\hline
\end{tabular}




\begin{tabular}{cccc}
$\mathrm{H}$ & 5.33861500 & 7.45362200 & 4.22212100 \\
$\mathrm{H}$ & 5.04712800 & 8.29260400 & 2.69786300 \\
$\mathrm{H}$ & 6.31613800 & 11.11514500 & 4.87311300 \\
$\mathrm{H}$ & 4.93409500 & 10.54606500 & 3.91847200 \\
$\mathrm{H}$ & 5.28272600 & 9.82407200 & 5.49167000 \\
$\mathrm{H}$ & 7.83949900 & 9.22621900 & 2.00486700 \\
$\mathrm{H}$ & 6.35666200 & 10.18989200 & 1.94127900 \\
$\mathrm{H}$ & 7.75953000 & 10.80155800 & 2.82599100 \\
$\mathrm{H}$ & 9.60170400 & 9.57589600 & 3.66206300 \\
$\mathrm{H}$ & 12.03826600 & 9.30889800 & 4.10285500 \\
$\mathrm{H}$ & 14.42006400 & 9.14793900 & 4.84749700 \\
$\mathrm{H}$ & 14.92633000 & 8.16618800 & 7.08903500 \\
$\mathrm{H}$ & 5.71776600 & 8.01717500 & 6.23746400 \\
$\mathrm{H}$ & 6.54515300 & 6.45161000 & 6.26159800 \\
$\mathrm{H}$ & 5.79064800 & 7.04389000 & 8.49981300 \\
$\mathrm{H}$ & 7.51199500 & 6.93793500 & 8.45257900 \\
$\mathrm{H}$ & 7.33375600 & 9.64569100 & 8.08334800 \\
$\mathrm{H}$ & 5.73741000 & 9.40650200 & 8.75856700 \\
$\mathrm{O}$ & 7.18949200 & 10.17121700 & 10.97587500 \\
$\mathrm{O}$ & 6.97149700 & 7.86401200 & 11.06056700 \\
$\mathrm{C}$ & 7.00643300 & 9.84552300 & 12.36474300 \\
$\mathrm{C}$ & 6.45202700 & 8.36288600 & 12.30624700 \\
$\mathrm{C}$ & 4.91715500 & 8.29660100 & 12.20832700 \\
$\mathrm{C}$ & 6.92579900 & 7.45133500 & 13.44188100 \\
$\mathrm{C}$ & 6.03432800 & 10.87159700 & 12.95841100 \\
$\mathrm{C}$ & 8.35688800 & 9.96747900 & 13.09342800 \\
$\mathrm{H}$ & 4.62428100 & 7.26611700 & 11.98102600 \\
$\mathrm{H}$ & 4.42855900 & 8.59538500 & 13.14291500 \\
$\mathrm{H}$ & 4.54550900 & 8.93786700 & 11.40275600 \\
$\mathrm{H}$ & 8.01529500 & 7.37244300 & 13.46353000 \\
$\mathrm{H}$ & 6.58152200 & 7.81761100 & 14.41680700 \\
$\mathrm{H}$ & 6.51727900 & 6.44500400 & 13.29716500 \\
$\mathrm{H}$ & 6.49708300 & 11.86429700 & 12.93836100 \\
$\mathrm{H}$ & 5.10634300 & 10.92499700 & 12.38420500 \\
$\mathrm{H}$ & 5.78985300 & 10.63346900 & 14.00070100 \\
$\mathrm{H}$ & 8.76317400 & 10.96765500 & 12.90959500 \\
& 8.24293600 & 9.83630900 & 14.17589500 \\
\hline & 9.08234700 & 9.23617000 & 12.72914000
\end{tabular}

(3-BBNNNtBu)Zn(SPh)(p-tBu-SPh) (No B-S interaction)

$\begin{array}{cccc}\text { Zn } & 9.11500300 & 7.63141600 & 8.94590600 \\ \text { S } & 8.20972300 & 9.31468900 & 10.21051100 \\ \text { S } & 8.55165800 & 5.47422100 & 9.46495500\end{array}$




\begin{tabular}{|c|c|c|c|}
\hline $\mathrm{N}$ & 11.15457800 & 7.94784800 & 8.53582800 \\
\hline $\mathrm{N}$ & 9.07724300 & 7.90982000 & 6.86270700 \\
\hline $\mathrm{N}$ & 8.19833100 & 7.82553700 & 5.84752300 \\
\hline C & 11.73929200 & 7.74199400 & 10.88624000 \\
\hline C & 12.13522400 & 7.98386100 & 9.45790800 \\
\hline C & 13.45892800 & 8.23572200 & 9.07830300 \\
\hline C & 13.75964100 & 8.45171300 & 7.73643300 \\
\hline C & 12.73825100 & 8.41172400 & 6.79123800 \\
\hline C & 11.43903700 & 8.15483400 & 7.22895800 \\
\hline C & 10.28876600 & 8.08084600 & 6.32233600 \\
\hline C & 10.18211300 & 8.12173200 & 4.92275400 \\
\hline C & 8.83055500 & 7.95386900 & 4.63388800 \\
\hline C & 8.17021000 & 7.87574600 & 3.26085300 \\
\hline C & 7.59995100 & 6.45716800 & 3.01148500 \\
\hline C & 9.24327200 & 8.14553700 & 2.18291900 \\
\hline C & 7.06302500 & 8.94462500 & 3.09433900 \\
\hline C & 6.77595500 & 7.67580400 & 6.19661700 \\
\hline C & 6.14346300 & 8.97609200 & 6.71386100 \\
\hline C & 4.61450700 & 8.85629500 & 6.84585600 \\
\hline C & 2.39550300 & 7.69133900 & 7.97765100 \\
\hline C & 2.13796700 & 6.16264000 & 7.82450500 \\
\hline C & 2.91874900 & 5.26371800 & 8.80638900 \\
\hline C & 4.40088400 & 5.65642900 & 8.97629200 \\
\hline C & 4.67081300 & 7.18465900 & 9.14010100 \\
\hline C & 4.11816600 & 7.79568200 & 10.46553800 \\
\hline C & 2.58001400 & 7.83878700 & 10.58670700 \\
\hline C & 1.85878900 & 8.30751200 & 9.30487800 \\
\hline C & 9.22806100 & 10.74458500 & 9.85802100 \\
\hline C & 9.82492200 & 11.44784400 & 10.91474200 \\
\hline C & 10.58456800 & 12.59786400 & 10.68573100 \\
\hline C & 10.79130200 & 13.10145800 & 9.39453000 \\
\hline C & 10.18851200 & 12.39295300 & 8.34044300 \\
\hline C & 9.42250800 & 11.25060900 & 8.56023800 \\
\hline C & 9.79726000 & 4.52531800 & 8.59069500 \\
\hline C & 9.77151300 & 4.36428300 & 7.19381900 \\
\hline C & 10.72754700 & 3.58151000 & 6.54379000 \\
\hline C & 11.72671700 & 2.93265800 & 7.27246300 \\
\hline C & 11.75661800 & 3.07481400 & 8.66152800 \\
\hline C & 10.80510300 & 3.86141000 & 9.31270500 \\
\hline B & 3.95961400 & 7.92341000 & 7.94474800 \\
\hline $\mathrm{H}$ & 8.95559300 & 10.75046200 & 7.71644300 \\
\hline $\mathrm{H}$ & 10.30645800 & 12.74374900 & 7.31761700 \\
\hline $\mathrm{H}$ & 11.01866000 & 13.10046800 & 11.54394600 \\
\hline $\mathrm{H}$ & 9.68496700 & 11.09096800 & 11.93142000 \\
\hline
\end{tabular}




$\begin{array}{cccc}\text { H } & 10.82715700 & 3.96001300 & 10.39434400 \\ \mathrm{H} & 12.52333000 & 2.56919000 & 9.24424600 \\ \mathrm{H} & 12.46648400 & 2.31840500 & 6.76577400 \\ \mathrm{H} & 10.68406800 & 3.47054500 & 5.46238200 \\ \mathrm{H} & 8.98450300 & 4.84516600 & 6.61993700 \\ \mathrm{H} & 5.75962900 & 7.31821500 & 9.16602300 \\ \mathrm{H} & 1.87004800 & 8.19395600 & 7.15128200 \\ \mathrm{H} & 1.95670600 & 9.40116400 & 9.22780500 \\ \mathrm{H} & 0.77998700 & 8.11134500 & 9.39810400 \\ \mathrm{H} & 2.20331900 & 6.85446100 & 10.87982100 \\ \mathrm{H} & 2.30788400 & 8.51157400 & 11.41137600 \\ \mathrm{H} & 4.53727000 & 7.24825400 & 11.32167100 \\ \mathrm{H} & 4.51228500 & 8.81842800 & 10.54862800 \\ \mathrm{H} & 2.42237500 & 5.26601700 & 9.78081300 \\ \mathrm{H} & 2.86418900 & 4.22411300 & 8.45520200 \\ \mathrm{H} & 4.83391300 & 5.11491300 & 9.82823200 \\ \mathrm{H} & 4.96162800 & 5.30474800 & 8.09717700 \\ \mathrm{H} & 1.06110500 & 5.95380100 & 7.91179300 \\ \mathrm{H} & 2.41225100 & 5.87824300 & 6.79650500 \\ \mathrm{H} & 12.61036900 & 7.75734000 & 11.54649700 \\ \mathrm{H} & 11.02661000 & 8.50615300 & 11.21741600 \\ \mathrm{H} & 11.23615200 & 6.77297400 & 10.98301700 \\ \mathrm{H} & 8.39036700 & 5.70267100 & 3.08732100 \\ \mathrm{H} & 6.81052200 & 6.18484800 & 3.71799200 \\ \mathrm{H} & 12.19219000 & 14.99762000 & 10.38771300 \\ \mathrm{H} & 7.17354800 & 6.40268800 & 2.00323100 \\ \mathrm{H} & 7.46010300 & 9.94988000 & 3.27337200 \\ \mathrm{H} & 6.67607700 & 8.91223500 & 2.06950300 \\ \mathrm{H} & 6.21607600 & 8.79233500 & 3.76781100 \\ \mathrm{H} & 10.04355400 & 7.39856700 & 2.21366700 \\ \mathrm{H} & 8.78264400 & 8.09810500 & 1.19066300 \\ \mathrm{H} & 9.68991200 & 9.13906300 & 2.29855000 \\ \mathrm{H} & 10.98039700 & 8.23702000 & 4.20742200 \\ \mathrm{H} & 12.93899800 & 8.57771500 & 5.73860300 \\ \mathrm{H} & 14.78220600 & 8.64980100 & 7.42806800 \\ \mathrm{H} & 14.23573300 & 8.25928400 & 9.83499200 \\ \mathrm{H} & 6.25817700 & 7.32050200 & 5.30629600 \\ \mathrm{H} & 6.72237300 & 6.88465600 & 6.95071700 \\ \mathrm{H} & 6.59171300 & 9.23060800 & 7.68093300 \\ \mathrm{H} & 4.40082800 & 9.78677500 & 6.01886200 \\ \mathrm{H} & 4.16159000 & 8.63610400 & 5.86375200 \\ \mathrm{H} & 11.62107100 & 14.36484800 & 9.10425300 \\ \mathrm{H} & & 15.42023000 & 8.40490000\end{array}$




$\begin{array}{cccc}\mathrm{C} & 12.80706500 & 14.00576000 & 8.17737300 \\ \mathrm{H} & 12.77243600 & 15.89253400 & 10.13436300 \\ \mathrm{H} & 11.39826200 & 15.30353300 & 11.07847400 \\ \mathrm{H} & 12.85982600 & 14.30963500 & 10.91917300 \\ \mathrm{H} & 10.32294900 & 15.04574400 & 7.46005300 \\ \mathrm{H} & 9.88608200 & 15.70314100 & 9.04307500 \\ \mathrm{H} & 11.30956000 & 16.32614800 & 8.18349100 \\ \mathrm{H} & 13.46431000 & 13.26803400 & 8.65246600 \\ \mathrm{H} & 12.46546300 & 13.58411300 & 7.22586500 \\ \mathrm{H} & 13.40411900 & 14.89849700 & 7.95196300\end{array}$

\section{(3-BBNNNtBu)Zn(SPh)(p-tBu-SPh) (B-S = $2.183 \AA$ Å)}

\begin{tabular}{|c|c|c|c|}
\hline $\mathrm{Zn}$ & 10.44261300 & 7.40581500 & 8.23058100 \\
\hline$S$ & 9.96507500 & 8.74452900 & 10.10451400 \\
\hline$S$ & 10.98174300 & 5.19280700 & 8.23192600 \\
\hline $\mathrm{N}$ & 11.90088200 & 8.34280000 & 7.00295200 \\
\hline$N$ & 9.28026300 & 7.96232900 & 6.57280200 \\
\hline $\mathrm{N}$ & 7.99953300 & 7.91462300 & 6.16648300 \\
\hline C & 13.69793100 & 7.92876600 & 8.59785900 \\
\hline C & 13.21585300 & 8.45764500 & 7.27689100 \\
\hline C & 14.08572200 & 9.05520700 & 6.35678600 \\
\hline C & 13.58642700 & 9.53596100 & 5.14986200 \\
\hline C & 12.22789900 & 9.41068700 & 4.87489800 \\
\hline C & 11.40907600 & 8.80418200 & 5.82820300 \\
\hline C & 9.97011300 & 8.61041900 & 5.62631500 \\
\hline C & 9.10879000 & 8.98487400 & 4.58372800 \\
\hline C & 7.84524200 & 8.52710800 & 4.94744800 \\
\hline C & 6.53919300 & 8.67419100 & 4.17274800 \\
\hline C & 5.90481700 & 7.29548500 & 3.86604000 \\
\hline C & 6.84324600 & 9.35585200 & 2.82020300 \\
\hline C & 5.54435900 & 9.58069100 & 4.93963500 \\
\hline C & 7.02877700 & 7.15758800 & 6.98572200 \\
\hline C & 7.25370500 & 7.32091100 & 8.49069000 \\
\hline C & 7.18619900 & 8.76179200 & 9.02063800 \\
\hline C & 7.65852700 & 10.46254500 & 11.14667700 \\
\hline C & 6.12838300 & 10.60788200 & 11.39127700 \\
\hline C & 5.47451300 & 9.46353300 & 12.20307300 \\
\hline C & 5.97569400 & 8.04138300 & 11.85586900 \\
\hline C & 7.51105900 & 7.90848000 & 11.63375900 \\
\hline C & 8.32534400 & 8.10877400 & 12.93559100 \\
\hline C & 8.32804900 & 9.54505700 & 13.50429600 \\
\hline C & 8.48335300 & 10.65743200 & 12.44368100 \\
\hline C & 10.69095700 & 10.32071400 & 9.63289900 \\
\hline
\end{tabular}




\begin{tabular}{|c|c|c|c|}
\hline C & 11.76144300 & 10.81769600 & 10.39231400 \\
\hline C & 12.36179600 & 12.03244000 & 10.06923000 \\
\hline C & 11.92178500 & 12.81119100 & 8.98535400 \\
\hline C & 10.84656200 & 12.30784400 & 8.24051200 \\
\hline C & 10.24047400 & 11.08765800 & 8.55277300 \\
\hline C & 10.14015300 & 4.42058000 & 9.61407200 \\
\hline C & 9.46508300 & 3.20798200 & 9.39675000 \\
\hline C & 8.84386900 & 2.53946200 & 10.45251800 \\
\hline C & 8.87672800 & 3.07090700 & 11.74340100 \\
\hline C & 9.54617100 & 4.27570900 & 11.96813500 \\
\hline C & 10.17936400 & 4.94208700 & 10.91733300 \\
\hline B & 7.88618100 & 8.99646000 & 10.48282100 \\
\hline $\mathrm{H}$ & 9.40873400 & 10.73788000 & 7.95047500 \\
\hline $\mathrm{H}$ & 10.45809700 & 12.86875600 & 7.39722800 \\
\hline $\mathrm{H}$ & 13.18758300 & 12.37862800 & 10.68521300 \\
\hline $\mathrm{H}$ & 12.11556300 & 10.24849400 & 11.24715400 \\
\hline $\mathrm{H}$ & 10.70775300 & 5.87109700 & 11.10980700 \\
\hline $\mathrm{H}$ & 9.58090100 & 4.70359000 & 12.96686000 \\
\hline $\mathrm{H}$ & 8.38755200 & 2.55308300 & 12.56410700 \\
\hline $\mathrm{H}$ & 8.32752500 & 1.60132600 & 10.26206700 \\
\hline $\mathrm{H}$ & 9.43170600 & 2.79453200 & 8.39281100 \\
\hline $\mathrm{H}$ & 7.68508200 & 6.86868300 & 11.31557000 \\
\hline $\mathrm{H}$ & 7.93859900 & 11.28179300 & 10.46505100 \\
\hline $\mathrm{H}$ & 9.54327200 & 10.73109900 & 12.16786100 \\
\hline $\mathrm{H}$ & 8.23302900 & 11.62411500 & 12.91028000 \\
\hline $\mathrm{H}$ & 7.41018500 & 9.71298300 & 14.07764300 \\
\hline $\mathrm{H}$ & 9.14235200 & 9.63657200 & 14.23801100 \\
\hline $\mathrm{H}$ & 7.96609600 & 7.42161200 & 13.71881900 \\
\hline $\mathrm{H}$ & 9.36366900 & 7.81505600 & 12.73251100 \\
\hline $\mathrm{H}$ & 5.62768500 & 9.64686700 & 13.27098100 \\
\hline $\mathrm{H}$ & 4.38459400 & 9.50255100 & 12.06055200 \\
\hline $\mathrm{H}$ & 5.64862100 & 7.35189500 & 12.65025200 \\
\hline $\mathrm{H}$ & 5.46391200 & 7.70180700 & 10.94368800 \\
\hline $\mathrm{H}$ & 5.91802800 & 11.56192900 & 11.90058200 \\
\hline $\mathrm{H}$ & 5.62840400 & 10.67968100 & 10.41569000 \\
\hline $\mathrm{H}$ & 14.78608500 & 7.99887800 & 8.67528900 \\
\hline $\mathrm{H}$ & 13.25579400 & 8.50498300 & 9.41898600 \\
\hline $\mathrm{H}$ & 13.39598700 & 6.88308300 & 8.72810700 \\
\hline $\mathrm{H}$ & 6.60701600 & 6.65593000 & 3.31989700 \\
\hline $\mathrm{H}$ & 5.59064200 & 6.75968800 & 4.76471500 \\
\hline $\mathrm{H}$ & 5.01683800 & 7.43471700 & 3.23900300 \\
\hline $\mathrm{H}$ & 5.97581100 & 10.57337500 & 5.10753300 \\
\hline $\mathrm{H}$ & 4.62883900 & 9.70308500 & 4.34961100 \\
\hline $\mathrm{H}$ & 5.25792900 & 9.17667800 & 5.91433800 \\
\hline
\end{tabular}




$\begin{array}{cccc}\text { H } & 7.53598700 & 8.76144300 & 2.21449800 \\ \mathrm{H} & 5.91340800 & 9.46742100 & 2.25280100 \\ \mathrm{H} & 7.26997100 & 10.35525700 & 2.95760400 \\ \mathrm{H} & 9.36100500 & 9.52312700 & 3.68438500 \\ \mathrm{H} & 11.80775400 & 9.76819700 & 3.94145400 \\ \mathrm{H} & 14.25035700 & 10.00142100 & 4.42700100 \\ \mathrm{H} & 15.14053400 & 9.13635000 & 6.59597500 \\ \mathrm{H} & 6.03956900 & 7.52251200 & 6.71515600 \\ \mathrm{H} & 7.10029300 & 6.10369700 & 6.69092200 \\ \mathrm{H} & 6.49287800 & 6.69985000 & 8.97960200 \\ \mathrm{H} & 8.20084200 & 6.83499800 & 8.75288200 \\ \mathrm{H} & 7.62968600 & 9.44801000 & 8.28502100 \\ \mathrm{H} & 6.12569700 & 9.05051900 & 9.06921100 \\ \mathrm{C} & 12.61439900 & 14.14724600 & 8.66223100 \\ \mathrm{C} & 12.51303300 & 15.09189100 & 9.88362300 \\ \mathrm{C} & 11.97966300 & 14.85921200 & 7.45241100 \\ \mathrm{C} & 14.10535500 & 13.88657200 & 8.33966200 \\ \mathrm{H} & 13.00613700 & 16.04803500 & 9.66887800 \\ \mathrm{H} & 11.46621300 & 15.29691500 & 10.13426000 \\ \mathrm{H} & 12.99007000 & 14.66346000 & 10.77118600 \\ \mathrm{H} & 12.04816300 & 14.25460700 & 6.54042200 \\ \mathrm{H} & 10.92419700 & 15.09684700 & 7.62709300 \\ \mathrm{H} & 12.50352400 & 15.80290000 & 7.26211900 \\ \mathrm{H} & 14.62785800 & 13.42037400 & 9.18169300 \\ \mathrm{H} & 14.20761500 & 13.22236000 & 7.47326600 \\ \mathrm{H} & 14.61826700 & 14.82844800 & 8.10871500\end{array}$

\section{(3-BBNNNtBu)Zn(SPh)( $p$ - $\left.\mathrm{CF}_{3}-\mathrm{SPh}\right)$ (No B-S interaction)}

$\begin{array}{cccc}\text { Zn } & 9.16871400 & 7.56261800 & 8.88796100 \\ \text { S } & 8.33666400 & 9.20871300 & 10.26561600 \\ \text { S } & 8.53745800 & 5.41942000 & 9.36144000 \\ \text { N } & 11.20172400 & 7.86365000 & 8.44622700 \\ \text { N } & 9.08632500 & 7.91337000 & 6.81950400 \\ \text { N } & 8.18562300 & 7.85936700 & 5.82072300 \\ \text { C } & 11.84277500 & 7.55427100 & 10.77213100 \\ \text { C } & 12.20391800 & 7.85853100 & 9.34644500 \\ \text { C } & 13.51996900 & 8.11832200 & 8.94631600 \\ \text { C } & 13.79231400 & 8.38104700 & 7.60682700 \\ \text { C } & 12.75028400 & 8.37752300 & 6.68346100 \\ \text { C } & 11.45966700 & 8.11246800 & 7.14051600 \\ \text { C } & 10.28977900 & 8.07499800 & 6.25681900 \\ \text { C } & 10.15512900 & 8.14158600 & 4.86103100 \\ \text { C } & 8.79513600 & 7.99904100 & 4.59719100\end{array}$




\begin{tabular}{|c|c|c|c|}
\hline C & 8.10603400 & 7.95648900 & 3.23657100 \\
\hline C & 7.50710200 & 6.55218500 & 2.97393300 \\
\hline C & 9.16172500 & 8.22816000 & 2.14203500 \\
\hline C & 7.01458500 & 9.04704500 & 3.11199200 \\
\hline C & 6.76820100 & 7.72429200 & 6.19534500 \\
\hline C & 6.16733900 & 9.02273500 & 6.75375400 \\
\hline C & 4.63821800 & 8.92712100 & 6.90278600 \\
\hline C & 2.41504800 & 7.75979500 & 8.02457900 \\
\hline C & 2.15186400 & 6.23383300 & 7.85303800 \\
\hline C & 2.93011700 & 5.32055400 & 8.82348900 \\
\hline C & 4.41403900 & 5.70545400 & 8.99643100 \\
\hline C & 4.69022600 & 7.23077000 & 9.17785100 \\
\hline C & 4.14108800 & 7.82790300 & 10.51107900 \\
\hline C & 2.60327300 & 7.87477000 & 10.63484500 \\
\hline C & 1.88197200 & 8.36144000 & 9.35982100 \\
\hline C & 9.23088000 & 10.69655500 & 9.86375200 \\
\hline C & 9.68780600 & 11.52056400 & 10.91033900 \\
\hline C & 10.36379000 & 12.70919300 & 10.64964000 \\
\hline C & 10.60427600 & 13.10927700 & 9.33160200 \\
\hline C & 10.14940100 & 12.30756100 & 8.27970700 \\
\hline C & 9.46689400 & 11.12362100 & 8.54228900 \\
\hline C & 9.85043900 & 4.46580400 & 8.59676600 \\
\hline C & 9.97382900 & 4.34572800 & 7.20115500 \\
\hline C & 10.98298500 & 3.56403800 & 6.63550600 \\
\hline C & 11.88566000 & 2.87542900 & 7.44883000 \\
\hline C & 11.76515000 & 2.97579000 & 8.83654200 \\
\hline C & 10.76077300 & 3.76139800 & 9.40446800 \\
\hline B & 3.97982000 & 7.98478500 & 7.99146200 \\
\hline $\mathrm{H}$ & 9.09674900 & 10.52638200 & 7.71525200 \\
\hline $\mathrm{H}$ & 10.31471400 & 12.62107600 & 7.25306900 \\
\hline $\mathrm{H}$ & 10.70442400 & 13.33025600 & 11.47221700 \\
\hline $\mathrm{H}$ & 9.50513100 & 11.21716600 & 11.93675100 \\
\hline $\mathrm{H}$ & 10.66502100 & 3.82829400 & 10.48453200 \\
\hline $\mathrm{H}$ & 12.45487500 & 2.43820500 & 9.48321600 \\
\hline $\mathrm{H}$ & 12.66691400 & 2.26242200 & 7.00722200 \\
\hline $\mathrm{H}$ & 11.05668800 & 3.48513600 & 5.55301400 \\
\hline $\mathrm{H}$ & 9.26190600 & 4.85831700 & 6.56042600 \\
\hline $\mathrm{H}$ & 5.77976000 & 7.36085400 & 9.20401100 \\
\hline $\mathrm{H}$ & 1.89060100 & 8.27430600 & 7.20501600 \\
\hline $\mathrm{H}$ & 1.98291800 & 9.45564300 & 9.29592300 \\
\hline $\mathrm{H}$ & 0.80281500 & 8.16735100 & 9.45209400 \\
\hline $\mathrm{H}$ & 2.22386900 & 6.88821400 & 10.91643900 \\
\hline $\mathrm{H}$ & 2.33464800 & 8.53826900 & 11.46808000 \\
\hline $\mathrm{H}$ & 4.55953400 & 7.26932000 & 11.36030400 \\
\hline
\end{tabular}




\begin{tabular}{|c|c|c|c|}
\hline $\mathrm{H}$ & 4.53801100 & 8.84869100 & 10.60586800 \\
\hline $\mathrm{H}$ & 2.43476000 & 5.31312600 & 9.79836800 \\
\hline $\mathrm{H}$ & 2.87155600 & 4.28543800 & 8.46011700 \\
\hline $\mathrm{H}$ & 4.84513900 & 5.15226200 & 9.84186700 \\
\hline $\mathrm{H}$ & 4.97267900 & 5.36162600 & 8.11304700 \\
\hline $\mathrm{H}$ & 1.07434300 & 6.02818800 & 7.93875900 \\
\hline $\mathrm{H}$ & 2.42415100 & 5.96077600 & 6.82146700 \\
\hline $\mathrm{H}$ & 12.72364200 & 7.58573400 & 11.41848000 \\
\hline $\mathrm{H}$ & 11.10105500 & 8.27122600 & 11.14234500 \\
\hline $\mathrm{H}$ & 11.38847400 & 6.55901100 & 10.84232200 \\
\hline $\mathrm{H}$ & 8.28544100 & 5.78301800 & 3.02241600 \\
\hline $\mathrm{H}$ & 6.72556000 & 6.28068700 & 3.68944000 \\
\hline $\mathrm{H}$ & 7.06227800 & 6.52255700 & 1.97271200 \\
\hline $\mathrm{H}$ & 7.43249000 & 10.04205900 & 3.30049400 \\
\hline $\mathrm{H}$ & 6.60689100 & 9.04001100 & 2.09481400 \\
\hline $\mathrm{H}$ & 6.17847200 & 8.89691700 & 3.79926500 \\
\hline $\mathrm{H}$ & 9.94991400 & 7.46779900 & 2.14276200 \\
\hline $\mathrm{H}$ & 8.68010600 & 8.20611200 & 1.15898900 \\
\hline $\mathrm{H}$ & 9.62692100 & 9.21215900 & 2.26557600 \\
\hline $\mathrm{H}$ & 10.94033400 & 8.25622400 & 4.13128400 \\
\hline $\mathrm{H}$ & 12.92883400 & 8.58005800 & 5.63325500 \\
\hline $\mathrm{H}$ & 14.80849700 & 8.58729900 & 7.28350000 \\
\hline $\mathrm{H}$ & 14.31349300 & 8.11101600 & 9.68568200 \\
\hline $\mathrm{H}$ & 6.22749500 & 7.39949800 & 5.30732400 \\
\hline $\mathrm{H}$ & 6.71567000 & 6.91562600 & 6.93078400 \\
\hline $\mathrm{H}$ & 6.63205500 & 9.24495900 & 7.72078800 \\
\hline $\mathrm{H}$ & 6.43065300 & 9.84600100 & 6.07624200 \\
\hline $\mathrm{H}$ & 4.25788500 & 9.92978300 & 7.17197800 \\
\hline $\mathrm{H}$ & 4.17054300 & 8.73737900 & 5.92154500 \\
\hline C & 11.39177900 & 14.35007000 & 9.03951100 \\
\hline $\mathrm{F}$ & 10.96307500 & 14.97064900 & 7.91380000 \\
\hline$F$ & 11.32548400 & 15.25014700 & 10.04682700 \\
\hline $\mathrm{F}$ & 12.71060900 & 14.08469700 & 8.84606400 \\
\hline
\end{tabular}

\section{(3-BBNNNtBu) $\mathrm{Zn}(\mathrm{SPh})\left(p-\mathrm{CF}_{3}-\mathrm{SPh}\right)(\mathrm{B}-\mathrm{S}=2.183 \AA$ A $)$}

$\begin{array}{cccc}\text { Zn } & 10.45909000 & 7.39025800 & 8.22414200 \\ \mathrm{~S} & 9.96371500 & 8.73390100 & 10.10355200 \\ \mathrm{~S} & 10.96789000 & 5.17525600 & 8.26655800 \\ \mathrm{~N} & 11.93040500 & 8.33564800 & 7.02339000 \\ \mathrm{~N} & 9.31356300 & 7.96659800 & 6.56791300 \\ \mathrm{~N} & 8.03595200 & 7.92314900 & 6.15011900 \\ \mathrm{C} & 13.71638300 & 7.88530100 & 8.62186500 \\ \mathrm{C} & 13.24423800 & 8.44146900 & 7.30823100\end{array}$




\begin{tabular}{|c|c|c|c|}
\hline C & 14.12181600 & 9.05341200 & 6.40548600 \\
\hline C & 13.63248500 & 9.55715400 & 5.20385300 \\
\hline C & 12.27611800 & 9.43762300 & 4.91555600 \\
\hline C & 11.44888600 & 8.81639000 & 5.85163200 \\
\hline C & 10.01135600 & 8.62641700 & 5.63505200 \\
\hline C & 9.15881600 & 9.01276600 & 4.58993300 \\
\hline C & 7.89222600 & 8.54975000 & 4.93706000 \\
\hline C & 6.59335600 & 8.70422200 & 4.15155900 \\
\hline C & 5.96515500 & 7.32800900 & 3.82202400 \\
\hline C & 6.90943900 & 9.40318800 & 2.81058700 \\
\hline C & 5.58937200 & 9.59942400 & 4.91982600 \\
\hline C & 7.05895800 & 7.15617400 & 6.95268600 \\
\hline C & 7.26732100 & 7.30849700 & 8.46135100 \\
\hline C & 7.18955700 & 8.74558500 & 9.00030900 \\
\hline C & 7.64574800 & 10.44226200 & 11.13290200 \\
\hline C & 6.11363400 & 10.57828400 & 11.37315900 \\
\hline C & 5.46268600 & 9.42727300 & 12.17781600 \\
\hline C & 5.97289800 & 8.00921100 & 11.82733000 \\
\hline C & 7.51026800 & 7.88566700 & 11.61230700 \\
\hline C & 8.31695200 & 8.08784100 & 12.91875300 \\
\hline C & 8.31041900 & 9.52288100 & 13.49037900 \\
\hline C & 8.46504300 & 10.63851100 & 12.43321000 \\
\hline C & 10.67837300 & 10.31054800 & 9.62765000 \\
\hline C & 11.76131400 & 10.80186000 & 10.37445900 \\
\hline C & 12.36169900 & 12.01634900 & 10.04749000 \\
\hline C & 11.87947500 & 12.76640800 & 8.97210800 \\
\hline C & 10.79207600 & 12.29512400 & 8.23105000 \\
\hline C & 10.19662800 & 11.07794000 & 8.55536900 \\
\hline C & 10.12782900 & 4.46558200 & 9.68472100 \\
\hline C & 9.32443900 & 3.32917000 & 9.49654200 \\
\hline C & 8.70216400 & 2.70994900 & 10.58159800 \\
\hline C & 8.86329800 & 3.21675900 & 11.87264400 \\
\hline C & 9.66163400 & 4.34573700 & 12.06848200 \\
\hline C & 10.29541600 & 4.96145500 & 10.98766500 \\
\hline B & 7.88120900 & 8.97905600 & 10.46611800 \\
\hline $\mathrm{H}$ & 9.35092900 & 10.72437600 & 7.97702500 \\
\hline $\mathrm{H}$ & 10.40470500 & 12.88549200 & 7.40660000 \\
\hline $\mathrm{H}$ & 13.19486300 & 12.38702100 & 10.63582100 \\
\hline $\mathrm{H}$ & 12.12307100 & 10.22918900 & 11.22293400 \\
\hline $\mathrm{H}$ & 10.92410100 & 5.83096100 & 11.15479000 \\
\hline $\mathrm{H}$ & 9.79674200 & 4.75219300 & 13.06755300 \\
\hline $\mathrm{H}$ & 8.37378300 & 2.73777800 & 12.71633500 \\
\hline $\mathrm{H}$ & 8.08502900 & 1.83019100 & 10.41436900 \\
\hline $\mathrm{H}$ & 9.19380700 & 2.93459000 & 8.49301300 \\
\hline
\end{tabular}




\begin{tabular}{|c|c|c|c|}
\hline $\mathrm{H}$ & 7.69232500 & 6.84790500 & 11.29240000 \\
\hline $\mathrm{H}$ & 7.92147000 & 11.26620600 & 10.45473500 \\
\hline $\mathrm{H}$ & 9.52635500 & 10.71770000 & 12.16276700 \\
\hline $\mathrm{H}$ & 8.20862000 & 11.60304000 & 12.90022400 \\
\hline $\mathrm{H}$ & 7.38930400 & 9.68583200 & 14.05965500 \\
\hline $\mathrm{H}$ & 9.12079200 & 9.61679800 & 14.22795000 \\
\hline $\mathrm{H}$ & 7.95634500 & 7.39742300 & 13.69810300 \\
\hline $\mathrm{H}$ & 9.35718600 & 7.79777500 & 12.72095600 \\
\hline $\mathrm{H}$ & 5.61021900 & 9.60723100 & 13.24699700 \\
\hline $\mathrm{H}$ & 4.37338100 & 9.46121900 & 12.03055900 \\
\hline $\mathrm{H}$ & 5.64698800 & 7.31491300 & 12.61772700 \\
\hline $\mathrm{H}$ & 5.46714300 & 7.66981200 & 10.91178700 \\
\hline $\mathrm{H}$ & 5.89757800 & 11.52913400 & 11.88551000 \\
\hline $\mathrm{H}$ & 5.61679900 & 10.65210900 & 10.39620700 \\
\hline $\mathrm{H}$ & 14.80355200 & 7.95633200 & 8.71014600 \\
\hline $\mathrm{H}$ & 13.26656800 & 8.44068100 & 9.45310200 \\
\hline $\mathrm{H}$ & 13.41563800 & 6.83649500 & 8.72737300 \\
\hline $\mathrm{H}$ & 6.67399400 & 6.69648400 & 3.27514200 \\
\hline $\mathrm{H}$ & 5.64288600 & 6.78075200 & 4.71090200 \\
\hline $\mathrm{H}$ & 5.08331000 & 7.47320600 & 3.18782900 \\
\hline $\mathrm{H}$ & 6.01677200 & 10.59105500 & 5.10355900 \\
\hline $\mathrm{H}$ & 4.67949700 & 9.72707100 & 4.32237400 \\
\hline $\mathrm{H}$ & 5.29400500 & 9.18315600 & 5.88671600 \\
\hline $\mathrm{H}$ & 7.60862000 & 8.81734900 & 2.20395700 \\
\hline $\mathrm{H}$ & 5.98487900 & 9.52057500 & 2.23586400 \\
\hline $\mathrm{H}$ & 7.33330200 & 10.40137400 & 2.96447200 \\
\hline $\mathrm{H}$ & 9.41848600 & 9.56132700 & 3.69900200 \\
\hline $\mathrm{H}$ & 11.86409100 & 9.81274800 & 3.98545200 \\
\hline $\mathrm{H}$ & 14.30217100 & 10.03704800 & 4.49615600 \\
\hline $\mathrm{H}$ & 15.17466300 & 9.13004800 & 6.65434100 \\
\hline $\mathrm{H}$ & 6.07154200 & 7.51967300 & 6.67408100 \\
\hline $\mathrm{H}$ & 7.13748500 & 6.10491400 & 6.65060500 \\
\hline $\mathrm{H}$ & 6.50329100 & 6.68192800 & 8.93791200 \\
\hline $\mathrm{H}$ & 8.21289500 & 6.82323100 & 8.72999600 \\
\hline $\mathrm{H}$ & 7.62755500 & 9.43882400 & 8.26784300 \\
\hline $\mathrm{H}$ & 6.12743700 & 9.02747500 & 9.04892100 \\
\hline C & 12.56199300 & 14.04361600 & 8.57259800 \\
\hline $\mathrm{F}$ & 13.16225200 & 14.64955400 & 9.61999000 \\
\hline $\mathrm{F}$ & 13.52814300 & 13.82501400 & 7.64394700 \\
\hline$F$ & 11.69880700 & 14.93003900 & 8.02755000 \\
\hline
\end{tabular}




\section{References}

${ }^{1}$ Polezhaev, A. V.; Chen, C.-H.; Losovyj, Y.; Caulton, K. G., A Multifunctional Pincer Ligand Supports Unsaturated Cobalt: Five Functionalities in One Pincer. Chem. Eur. J. 2017, 23, 8039-8050.

2 Toure, M.; Chuzel, O.; Parrain, J.-L. Synthesis and structure of Ag(i), Pd(ii), Rh(i), Ru(ii) and Au(i) NHC-complexes with a pendant Lewis acidic boronic ester moiety. Dalton Trans. 2015, 44, 7139-7143.

${ }^{3}$ Kiernicki, J. J.; Norwine, E. E.; Lovasz, M. L.; Zeller, M.; Szymczak, N. K., Mobility of Lewis Acids within the Secondary Coordination Sphere: Toward a Model for Cooperative Substrate Binding. Chem. Commun. 2020, 56, 13105-13108.

${ }^{4}$ Kiernicki, J. J.; Norwine, E. E.; Zeller, M.; Szymczak, N. K., Tetrahedral Iron Featuring an Appended Lewis Acid: Distinct Pathways for the Reduction of Hydroxylamine and Hydrazine Chem. Commun. 2019, 55, 11896-11899.

${ }^{5}$ Bruker Advanced X-ray Solution, Apex3, SAINT, SADABS, Bruker AXS Inc.: Madison (WI), USA, 2018.

${ }^{6}$ SHELXTL suite of programs, Version 6.14, 2000-2003, Bruker Advanced X-ray Solutions, Bruker AXS Inc., Madison, Wisconsin: USA

${ }^{7}$ Sheldrick, G. M., A Short History of SHELX. Acta Crystallogr., Sect. A. 2008, 64, 112-122.

${ }^{8}$ Sheldrick, G.M. Crystal structure refinement with SHELXL. Acta Crystallogr. Sect. C Struct. Chem. 2015, 71, 3-8.

${ }^{9}$ Hübschle, C.B., Sheldrick, G.M., Dittrich, B. ShelXle: a Qt graphical user interface for SHELXL. J. Appl. Crystallogr. 2011, 44, 1281-1284.

${ }^{10}$ Gaussian 09, Revision D.01, M. J. Frisch, G. W. Trucks, H. B. Schlegel, G. E. Scuseria, M. A. Robb, J. R. Cheeseman, G. Scalmani, V. Barone, G. A. Petersson, H. Nakatsuji, X. Li, M. Caricato, A. Marenich, J. Bloino, B. G. Janesko, R. Gomperts, B. Mennucci, H. P. Hratchian, J. V. Ortiz, A. F. Izmaylov, J. L. Sonnenberg, D. Williams-Young, F. Ding, F. Lipparini, F. Egidi, J. Goings, B. Peng, A. Petrone, T. Henderson, D. Ranasinghe, V. G. Zakrzewski, J. Gao, N. Rega, G. Zheng, W. Liang, M. Hada, M. Ehara, K. Toyota, R. Fukuda, J. Hasegawa, M. Ishida, T. Nakajima, Y. Honda, O. Kitao, H. Nakai, T. Vreven, K. Throssell, J. A. Montgomery, Jr., J. E. Peralta, F. Ogliaro, M. Bearpark, J. J. Heyd, E. Brothers, K. N. Kudin, V. N. Staroverov, T. Keith, R. Kobayashi, J. Normand, K. Raghavachari, A. Rendell, J. C. Burant, S. S. Iyengar, J. Tomasi, M. Cossi, J. M. Millam, M. Klene, C. Adamo, R. Cammi, J. W. Ochterski, R. L. Martin, K. Morokuma, O. Farkas, J. B. Foresman, and D. J. Fox, Gaussian, Inc., Wallingford CT, 2016.

${ }^{11}$ Stephens, P. J.; Devlin, F. J.; Chabalowski, C. F.; Frisch, M. J. Ab Initio Calculation of Vibrational Absorption and Circular Dichroism Spectra using Density Functional Force Fields. J. Phys. Chem. 1994, 98, 11623-11627.

${ }^{12}$ Rassolov, V. A.; Pople, J. A.; Ratner, M. A.; Windus, T. L. 6-31G* Basis Set for Atoms K through Zn. J. Chem. Phys. 1998, 109, 1223-1229.

${ }^{13}$ Eller, C.; Kehr, G.; Daniliuc, C. D.; Fröhlich, R.; and Erker, G. Facile 1,1-Carboboration Reactions of Acetylenic Thioethers. Organometallics 2013, 32, 384-386.

${ }^{14}$ Carey, F. A. S., Richard J., Advanced Organic Chemistry Part A: Structure and Mechanism. 5th ed.; Springer: 2007. 